\title{
$L$-functions of symmetric powers of the generalized Airy family of exponential sums
}

\author{
C. Douglas Haessig* $\quad$ Antonio Rojas-León ${ }^{\dagger}$
}

December 7, 2010

\begin{abstract}
This paper looks at the $L$-function of the $k$-th symmetric power of the $\overline{\mathbb{Q}}_{\ell}$-sheaf $\mathrm{Ai}_{f}$ over the affine line $\mathbb{A}_{\mathbb{F}_{q}}^{1}$ associated to the generalized Airy family of exponential sums. Using $\ell$-adic techniques, we compute the degree of this rational function as well as the local factors at infinity. Using $p$-adic techniques, we study the $q$-adic Newton polygon of these $L$-function.
\end{abstract}

\section{Introduction}

In this paper we study the $L$-function attached to the $k$-th symmetric power of the $\overline{\mathbb{Q}}_{\ell}$-sheaf $\mathrm{Ai}_{f}$ associated to the generalized Airy family of exponential sums. Symmetric powers appear in the proofs of many arithmetic problems. For instance, Deligne's proof [6] of the Ramanujan-Petersson conjecture relies on the construction of a Galois module coming from the $k$-th symmetric power of a certain $\ell$-adic sheaf. The Sato-Tate conjecture [5] [16] 27] relies on the analytic continuation of the $L$-function attached to the $k$-th symmetric power of an $\ell$-adic representation coming from an elliptic curve. Another equidistribution result concerning Kloosterman angles was proven by Adolphson [4 using results of Robba's 23 on the $L$-function of the $k$-th symmetric power of the $\ell$-adic Kloosterman sheaf $\mathrm{Kl}_{2}$. Symmetric powers also arise in the proof of Dwork's conjecture [29] [30] [31]. To begin, let us recall the general setup of an $L$-function of an $\ell$-adic representation.

Let $\mathbb{F}_{q}$ be the finite field of $q$ elements and characteristic $p$. Let $Y$ be a smooth, geometrically connected, open variety defined over $\mathbb{F}_{q}$; for instance, take $Y$ to be affine $s$-space $\mathbb{A}_{\mathbb{F}_{q}}^{s}$ or the torus $\mathbb{G}_{m}^{s}$. Denote its function field by $K$, and its corresponding absolute Galois group by $G_{K}:=\operatorname{Gal}\left(K^{\text {sep }} / K\right)$. Let $V$ be a finite dimensional vector space over a finite extension field of $\mathbb{Q}_{\ell}$, where $\ell \neq p$. Let $\rho: G_{K} \rightarrow G L(V)$ be a continuous $\ell$-adic representation unramified on $Y$, and let $\mathcal{F}$ be the corresponding lisse sheaf on $Y$. Define the $L$-function of $\rho$ on $Y$ by

$$
L(Y, \rho, T):=\prod_{x \in|Y|} \frac{1}{\operatorname{det}\left(1-\rho\left(\operatorname{Frob}_{x}\right) T^{\operatorname{deg}(x)}\right)} .
$$

By the Lefschetz trace formula, this is a rational function whose zeros and poles may be described using étale cohomology with compact support:

$$
L(Y, \rho, T)=\prod_{i=0}^{2 \operatorname{dim}(Y)} \operatorname{det}\left(1-\operatorname{Frob}_{q} T \mid H_{c}^{i}\left(Y \otimes \overline{\mathbb{F}}_{q}, \mathcal{F}\right)\right)^{(-1)^{i+1}}
$$

Given such a representation, we may construct new $L$-functions via operations such as tensor, symmetric, or exterior products. Natural questions about these new $L$-functions concern the determination of their degrees (Euler characteristic) and describing various properties about their zeros and poles. In this paper, we will focus on the symmetric powers of a particular family of exponential sums called the generalized Airy family. Other families whose symmetric powers have been investigated are the Legendre family of elliptic curves [3] [10] and the hyperKloosterman family [12 [13 [23. We note that the former seems to have been motivated by Dwork's $p$-adic interest in the Ramanujan-Petersson conjecture.

The generalized Airy family is defined as follows. Let $f$ be a polynomial over $\mathbb{F}_{q}$ of degree $d$ with $p \nmid d$. Let $\psi$ be a nontrivial additive character on $\mathbb{F}_{q}$. For each $\bar{t} \in \overline{\mathbb{F}}_{q}$ define its degree by $\operatorname{deg}(\bar{t}):=\left[\mathbb{F}_{q}(\bar{t}): \mathbb{F}_{q}\right]$. It is well-known that the associated $L$-function of the sequence of exponential sums

$$
S_{m}(\bar{t}):=\sum_{x \in \mathbb{F}_{q^{m} \operatorname{deg}(\bar{t})}} \psi \circ \operatorname{Tr}_{\mathbb{F}_{q^{m} \operatorname{deg}(\bar{t})} / \mathbb{F}_{q}}(f(x)+\bar{t} x) \quad \text { for } m=1,2,3, \ldots
$$

*Partially supported by NSF grant DMS-0901542

†Partially supported by PO8-FQM-03894 (Junta de Andalucía), MTM2007-66929 and FEDER 
is a polynomial of degree $d-1$ :

$$
L\left(f, \mathbb{A}^{1}, \bar{t} ; T\right):=\exp \left(\sum_{m=1}^{\infty} S_{m}(\bar{t}) \frac{T^{m}}{m}\right)=\left(1-\pi_{1}(\bar{t}) T\right) \cdots\left(1-\pi_{d-1}(\bar{t}) T\right) .
$$

As we will describe later, the relative cohomology of this family may be represented $\ell$-adically as a lisse sheaf of rank $d-1$ over $\mathbb{A}^{1}$ via Fourier transform. Let us denote this sheaf by $\mathrm{Ai}_{f}$. The $L$-function of the $k$-th symmetric power of $\mathrm{Ai}_{f}$ takes the form:

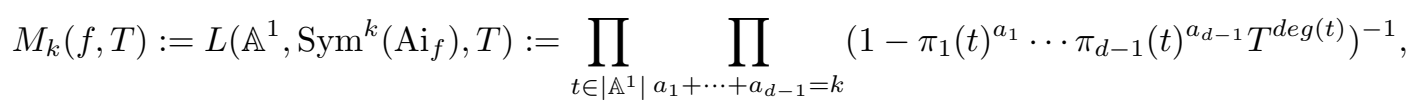

where $\left|\mathbb{A}^{1}\right|$ denotes the set of closed points on $\mathbb{A}^{1}$. By the Lefschetz trace formula, $M_{k}(f, T)$ is a rational function. The $\ell$-adic sheaf $\mathrm{Ai}_{f}$ was extensively studied by N. Katz in [18, where its monodromy group is determined and, as a consequence, an equidistribution result is obtained for the exponential sums in the family ([18, Corollary 20]). From these results it follows that, for $p>2 d-1, M_{k}(f, T)$ is in fact a polynomial. For $d=3$, a study of the monodromy group may be avoided using Adolphson's method 4 .

Our first main result is the computation of the degree of $M_{k}(f, T)$ for $p>d$. The degree of the rational function $M_{k}(f, T)$ equals the $k$-th coefficient of a generating series which is explicitly given in Corollary 3.4 . Simplified formulas are given in section 5 for some particularly nice values of $f$ and $p$.

As an example of this theorem, consider the family generated by $f(x)=x^{d}$. Then the degree of $M_{k}\left(x^{d}, T\right)$ may be described as follows. Let $\zeta$ be a primitive $(d-1)$-th root of unity in $\overline{\mathbb{F}}_{q}$. Denote by $N_{d-1, k}$ the number of $(d-1)$ tuples $\left(a_{0}, a_{1}, \ldots, a_{d-2}\right)$ of nonnegative integers such that $a_{0}+a_{1}+\cdots+a_{d-2}=k$ and $a_{0}+a_{1} \zeta+\cdots+a_{d-2} \zeta^{d-2}=0$ in $\overline{\mathbb{F}}_{q}$.

Theorem 1.1. With the notation defined above, we have

$$
\operatorname{deg} M_{k}\left(x^{d}, T\right)=\frac{1}{d-1}\left[\left(\begin{array}{c}
k+d-2 \\
d-2
\end{array}\right)-d N_{d-1, k}\right] .
$$

It was conjectured in [15] that $M_{k}\left(x^{3}, T\right)$ is a polynomial for all $p>3$ since it was shown, in that paper, that $M_{k}\left(x^{3}, T\right)$ is a polynomial for every odd integer $k$, and also for every $k$ even with $k<2 p$. Surprisingly, for $p=5$, $M_{k}\left(x^{3}, T\right)$ is not a polynomial for infinitely many $k$. This was communicated to the first author by N. Katz and is a consequence of the geometric monodromy group of $\mathrm{Ai}_{x^{3}}$ being finite.

Theorem 1.2. Suppose $p>2 d-1$. Then $M_{k}(f, T)$ is a polynomial which may be factored into a product $Q_{k}(f, T) P_{k}(f, T)$, where $P_{k}(f, T)$ satisfies the functional equation

$$
P_{k}(f, T)=c T^{\operatorname{deg}\left(P_{k}\right)} \overline{P_{k}\left(f, 1 / q^{k+1} T\right)} \quad \text { with }|c|=q^{\operatorname{deg}\left(P_{k}\right)(k+1) / 2}
$$

and $Q_{k}(f, T)$ has reciprocal roots of weight $\leq k$. Furthermore, writing $f(x)=\sum_{i=0}^{d} c_{i} x^{i}$, if we assume $\mathbb{F}_{q}$ contains the $2(d-1)$-th roots of $-d c_{d}$ then an explicit description of $Q_{k}(f, T)$ may be given; see Corollary 4.3 .

Lastly, we wish to describe the $p$-adic behavior of the reciprocal roots of $M_{k}(f, T)$. Motivation for such a study comes from Wan's reciprocity theorem [28] of the Gouvêa-Mazur conjecture [14] on the slopes of modular forms; see [3] for the connection between symmetric powers of the Legendre crystal with Hecke polynomials. Now, while we study in Section 6 the $q$-adic Newton polygon of the $L$-function for general $f$ and $k$, our most precise results occur in the cubic case $f(x)=x^{3}$ :

Theorem 1.3. Assume $p \geq 7, k$ is odd, and $k<p$. Write

$$
M_{k}\left(x^{3}, T\right)=1+c_{1} T+\cdots+c_{r} T^{r}
$$

Then

$$
\operatorname{ord}_{q}\left(c_{m}\right) \geq \frac{1}{3}\left(m^{2}+m+k m\right) \quad \text { for } m=0,1,2, \ldots, r .
$$

Furthermore, as a consequence of the functional equation, the endpoints of the q-adic Newton polygon of $M_{k}\left(x^{3}, T\right)$ coincide with the lower bound (2). If $p=5$, then the numerator of $M_{k}\left(x^{3}, T\right)$ satisfies (2).

We are hopeful that the restrictions $k$ odd and $k<p$ may be removed from the theorem (see [15] for details). There is also reason to believe that the lower bound (2) may be optimal in the sense that the $q$-adic Newton polygon will in fact equal this lower bound under certain conditions on $p$ and $k$. As supporting evidence we note two facts. First, as mentioned in the above theorem, the endpoints of the $q$-adic Newton polygon of $M_{k}\left(x^{3}, T\right)$ 
and the lower bound coincide. Secondly, the lower bound has the following symmetric property. Consider the points $P_{m} \in \mathbb{R}^{2}$ defined by the lower bound:

$$
P_{m}:=\left(m, \frac{1}{3}\left(m^{2}+m+k m\right)\right)
$$

The slope of the line segment joining $P_{m}$ and $P_{m+1}$ is given by $s_{m}:=\frac{1}{3}(2 m+2+k)$. If we set $m^{\prime}:=\frac{k-1}{2}-m$, then we have the symmetry

$$
(k+1)-s_{m}=s_{m^{\prime}}
$$

In other words, for every slope $s_{m}$ there is a corresponding slope $s_{m^{\prime}}$. This is precisely a consequence of the functional equation for $M_{k}\left(x^{3}, T\right)$. That is, if $\alpha$ is a reciprocal root of $M_{k}\left(x^{3}, T\right)$ of slope $s$, then $p^{k+1} / \alpha$ is another reciprocal root whose slope is $(k+1)-s$.

Acknowledgments. We would like to thank Nicholas Katz and Steven Sperber for their very helpful comments.

\section{Cohomological interpretation of $M_{k}(f, T)$}

In this section we will study the generalized Airy family of exponential sums from the point of view of $\ell$-adic cohomology. We will do so by studying the sheaf $\mathrm{Ai}_{f}$ that represents this family on the affine line $\mathbb{A}^{1}$ over the given finite field $\mathbb{F}_{q}$. We begin by observing that the map $\mathbb{F}_{q} \rightarrow \mathbb{C}$ given by $t \mapsto \sum_{x \in \mathbb{F}_{q}} \psi(f(x)+t x)$ is the Fourier transform with respect to $\psi$, in the classical sense, of the map $t \mapsto \psi(f(t))$. This will translate, in the cohomological sense, to the fact that $\mathrm{Ai}_{f}$ is the Fourier transform, in the sheaf-theoretical sense, of the $\overline{\mathbb{Q}}_{\ell}$-sheaf that represents the latter map, which is just the pull-back of the Artin-Schreier sheaf associated to $\psi$ via the map given by $f$. Let us be more precise.

The polynomial $f$ naturally defines a morphism, also denoted by $f: \mathbb{A}_{\mathbb{F}_{q}}^{1} \rightarrow \mathbb{A}_{\mathbb{F}_{q}}^{1}$. Let $\mathcal{L}_{\psi}$ be the Artin-Schreier sheaf on $\mathbb{A}_{\mathbb{F}_{q}}^{1}$ associated to $\psi$ (cf. [7, 1.7]). For every finite extension $\mathbb{F}_{q^{m}}$ of $\mathbb{F}_{q}$, every $t \in \mathbb{A}^{1}\left(\mathbb{F}_{q^{m}}\right)=\mathbb{F}_{q^{m}}$ and

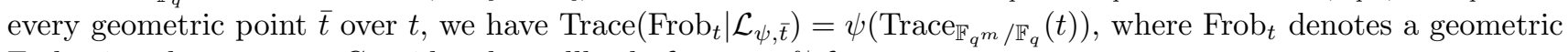
Frobenius element at $t$. Consider the pullback $\mathcal{L}_{\psi(f)}:=f^{\star} \mathcal{L}_{\psi}$.

By [18, Theorem 17], for $d \geq 2$ the Fourier transform with respect to $\psi$ of $\mathcal{L}_{\psi(f)}$ (which, in principle, is an element of the derived category $\left.\mathcal{D}_{c}^{b}\left(\mathbb{A}^{1}, \overline{\mathbb{Q}}_{\ell}\right)\right)$ is in fact a (shifted) lisse sheaf on $\mathbb{A}^{1}$, of rank $d-1$ and with $d /(d-1)$ as its single slope at infinity. Its Swan conductor is therefore $d$. Let us denote this sheaf by $\mathrm{Ai}_{f}=\mathrm{R}^{1} \pi_{t !} \mathcal{L}_{\psi(f(x)+t x)}$, where $\pi_{t}: \mathbb{A}^{2} \rightarrow \mathbb{A}^{1}$ is the projection $(x, t) \mapsto t$. For every finite extension $\mathbb{F}_{q^{m}}$ of $\mathbb{F}_{q}$, every $t \in \mathbb{F}_{q^{m}}$ and every geometric point $\bar{t}$ over $t$ we have, denoting $\psi_{m}=\psi \circ \operatorname{Trace}_{\mathbb{F}_{q} / \mathbb{F}_{q}}$ :

$$
\operatorname{Trace}\left(\operatorname{Frob}_{t} \mid\left(\mathrm{Ai}_{f}\right)_{\bar{t}}\right)=-\sum_{x \in \mathbb{F}_{q} m} \psi_{m}(f(x)+t x)
$$

The characteristic polynomial of the action of a geometric Frobenius element Frob $\mathrm{b}_{t}$ at $t$ on the stalk of $\mathrm{Ai}_{f}$ at a geometric point over $t$ has the form

$$
L\left(\mathrm{Ai}_{f}, t, T\right)=\left(1-\pi_{1}(t) T\right) \cdots\left(1-\pi_{d-1}(t) T\right)
$$

where $\pi_{i}(t)$ is a Weil algebraic number of weight 1 (i.e. all its complex conjugates have absolute value $q^{1 / 2}$ ) and $\sum_{x \in \mathbb{F}_{q} m} \psi_{m}(f(x)+t x)=-\sum_{i} \pi_{i}(t)^{m}$ for all $m \geq 1$. Its $k$-th "symmetric power" is given by

$$
L\left(k ; \mathrm{Ai}_{f}, t, T\right):=\prod_{a_{1}+\cdots+a_{d-1}=k}\left(1-\pi_{1}(t)^{a_{1}} \cdots \pi_{d-1}(t)^{a_{d-1}} T\right) .
$$

These are the local factors of the $L$-function of the $k$-th symmetric power of $\mathrm{Ai}_{f}$, which is given by the infinite product

$$
M_{k}(f, T):=\prod_{t \in\left|\mathbb{A}^{1}\right|} L\left(k ; \mathrm{Ai}_{f}, t, T^{\operatorname{deg}(t)}\right)^{-1}
$$

The Lefschetz trace formula demonstrates that the zeros and poles of $M_{k}(f, T)$ may be described in terms of cohomology:

$$
M_{k}(f, T)=\prod_{i=0}^{2} \operatorname{det}\left(1-\operatorname{Frob} T \mid \mathrm{H}_{c}^{i}\left(\mathbb{A}_{\overline{\mathbb{F}}_{q}}^{1}, \operatorname{Sym}^{k} \mathrm{Ai}_{f}\right)\right)^{(-1)^{i+1}} .
$$


Since $\operatorname{Sym}^{k} \mathrm{Ai}_{f}$ is a lisse sheaf on the affine line, we have $\mathrm{H}_{c}^{0}\left(\mathbb{A}_{\mathbb{F}_{q}}, \operatorname{Sym}^{k} \mathrm{Ai}_{f}\right)=0$, and the previous formula simplifies to

$$
M_{k}(f, T)=\frac{\operatorname{det}\left(1-\operatorname{Frob} T \mid \mathrm{H}_{c}^{1}\left(\mathbb{A}_{\overline{\mathbb{F}}_{q}}^{1}, \operatorname{Sym}^{k} \mathrm{Ai}_{f}\right)\right)}{\operatorname{det}\left(1-\operatorname{Frob} T \mid \mathrm{H}_{c}^{2}\left(\mathbb{A}_{\mathbb{F}_{q}}^{1}, \operatorname{Sym}^{k} \mathrm{Ai}_{f}\right)\right)} .
$$

On the other hand, $\mathrm{H}_{c}^{2}\left(\mathbb{A}_{\mathbb{F}_{q}}^{1}\right.$, $\left.\operatorname{Sym}^{k} \mathrm{Ai}_{f}\right)$ is just the space of co-invariants of the sheaf $\mathrm{Sym}^{k} \mathrm{Ai}_{f}$, regarded as a representation of the fundamental group $\pi_{1}\left(\mathbb{A}_{\mathbb{F}_{q}}^{1}\right)$, which is the $k$-th symmetric power of $\mathrm{Ai}_{f}$ regarded as a representation of the same group. This is the same as the space of co-invariants for its monodromy group, which is defined to be the Zariski closure of its image in the group of automorphisms of the generic stalk of $\mathrm{Ai}_{f}$, isomorphic to $\mathrm{GL}(d-1):=\mathrm{GL}\left(d-1, \overline{\mathbb{Q}}_{\ell}\right)$. By [18, Theorem 19], for $p>2 d-1$ the geometric monodromy group of $\mathrm{Ai}_{f}$ is either $\mathrm{SL}(d-1)$ for $d$ even, or $\mathrm{Sp}(d-1)$ for $d$ odd if $c_{d-1}=0$ and $\mu_{p} \cdot \mathrm{SL}(d-1)$ for $d$ even or $\mu_{p} \cdot \operatorname{Sp}(d-1)$ for $d$ odd if $c_{d-1} \neq 0$ (where $f(x)=\sum_{i=0}^{d} c_{i} x^{i}$ ). In either case, its $k$-th symmetric power is still an irreducible representation of rank $\left(\begin{array}{c}d+k-2 \\ d-2\end{array}\right)$ of the monodromy group (because it is an irreducible representation of its subgroup $\mathrm{SL}(d-1)$ or $\mathrm{Sp}(d-1))$, and in particular the space of co-invariants vanishes. More generally, it was proven by O. Such ([26, Proposition 1.6]) that, for $p>2$, either $\mathrm{Ai}_{f}$ has finite monodromy or its monodromy group contains $\mathrm{SL}(d-1)$ or $\mathrm{Sp}(d-1)$. In order to rule out the finite monodromy case for $p \leq 2 d-1$ one may use for instance [20, Proposition 8.14.3], which implies that $\mathrm{Ai}_{f}$ has finite monodromy if and only if for every element $t \in \overline{\mathbb{F}_{q}}$ the Newton polygon of the $L$-function associated to the exponential sum $\sum \psi(f(x)+t x)$ has a single slope.

Consequently, we have the following:

Theorem 2.1. If $\mathrm{Ai}_{f}$ does not have finite monodromy (e.g. if $p>2 d-1$ ), the L-function of the $k$-th symmetric power of $\mathrm{Ai}_{f}$ is a polynomial:

$$
M_{k}(f, T)=\operatorname{det}\left(1-\operatorname{Frob} T \mid \mathrm{H}_{c}^{1}\left(\mathbb{A}_{\mathbb{F}_{q}}^{1}, \operatorname{Sym}^{k} \mathrm{Ai}_{f}\right)\right)
$$

While it is tempting to believe that $M_{k}(f, T)$ is always a polynomial, this is not true, as mentioned in the introduction. In fact, the monodromy group can be finite in certain cases; for instance when $p=5$ and $f(x)=x^{3}$, as proven in 21]. In such cases, $\mathrm{H}_{c}^{2}\left(\mathbb{A}_{\mathbb{F}_{q}}^{1}, \operatorname{Sym}^{k} \mathrm{Ai}_{f}\right)$ will be non-trivial for infinitely many values of $k$, and consequently $M_{k}(f, T)$ will have a denominator.

Remark 2.2. Arithmetic difficulties often arise when the characteristic $p$ is small compared to $d$, as demonstrated above by the link between the finiteness of the monodromy group when $p \leq 2 d-1$ and the Newton polygons of the fibres of the family. By the functional equation, if we denote by $\mathrm{NP}_{1}(t)$ the slope of the first line segment of the Newton polygon of the fibre $t$ then $\mathrm{NP}_{1}(t) \leq 1 / 2$ with equality if and only if the Newton polygon is a single line segment. If $p \equiv 1$ modulo $d$, and in particular when $p=d+1$, then by [25, Theorem 3.11] the Newton polygon of every fibre equals the $q$-adic Newton polygon of the polynomial $\prod_{i=1}^{d-1}\left(1-q^{i / d} T\right)$. Thus, $\operatorname{NP}_{1}(t)=1 / d$ and so the monodromy group is infinite when $p=d+1>3$.

Let $[f(x)]_{x^{N}}$ denote the coefficient of $x^{N}$ in $f(x)$. Suppose $\frac{d}{2}+1<p \leq 2 d-1$ and $f$ has coefficients over $\mathbb{F}_{p}$. By [24, Theorem 2], if $\left[(f(x)+t x)^{\left\lceil\frac{p-1}{d}\right\rceil}\right]_{x^{p-1}} \not \equiv 0$ modulo $p$ for some $0 \leq t \leq p-1$, then $\operatorname{NP}_{1}(t) \leq\left\lceil\frac{p-1}{d}\right\rceil /(p-1)$ for those $t$. By the assumption on $d$ and $p$, notice that $\left\lceil\frac{p-1}{d}\right\rceil /(p-1)$ equals either $1 /(p-1)$ or $2 /(p-1)$. Hence, the mondromy group is infinite when such a $t$ exists and $p \geq 7$. Their argument may be extended as follows.

Let $d>p-1$. For a polynomial $h(x)$, define $(h(x))_{s}:=h(x)(h(x)-1) \cdots(h(x)-s+1)$. Define the linear operator $U: \mathbb{F}_{p}[x] \rightarrow \mathbb{F}_{p}$ by linearly extending the map which sends monomials $x^{n}$ to 0 if $(p-1) \nmid n$ and 1 otherwise. Let $c_{s}:=U\left((f(x)+t x)_{s}\right) \in \mathbb{F}_{p}$. Suppose $c_{1} \equiv \cdots \equiv c_{k-1} \equiv 0$ modulo $p$ and $c_{k} \not \equiv 0$ mod $p$ for some $t$, then $\mathrm{NP}_{1}(t) \leq \frac{k}{p-1}$. Hence, if this happens for some $k<(p-1) / 2$ then the mondromy group is infinite. For example, for $d>p-1$ and $f(x)=x^{d}+x^{p-1}$ then $c_{1}=1$ and hence the monodromy group is infinite for $p \geq 5$.

Lastly, we mention the case when $d=4, p=7$ and $f \in \mathbb{F}_{q}[x]$ is not of the form $(x+a)^{4}+b x+c$. Then by [17, Theorem 4.6] the monodromy of $\mathrm{Ai}_{f}$ is infinite.

\section{Computation of the degree of the $L$-function}

We will now study the degree of $M_{k}(f, T)$ when $p>d$. From the formula above we have

$$
\operatorname{deg}\left(M_{k}(f, T)\right)=\operatorname{dim}\left(\mathrm{H}_{c}^{1}\left(\mathbb{A}_{\mathbb{F}_{q}}^{1}, \operatorname{Sym}^{k} \operatorname{Ai}_{f}\right)\right)-\operatorname{dim}\left(\mathrm{H}_{c}^{2}\left(\mathbb{A}_{\mathbb{F}_{q}}^{1}, \operatorname{Sym}^{k} \mathrm{Ai}_{f}\right)\right)=-\chi_{c}\left(\mathbb{A}_{\mathbb{F}_{q}}^{1}, \operatorname{Sym}^{k} \mathrm{Ai}_{f}\right),
$$


where $\chi_{c}$ denotes the Euler characteristic with compact supports. Using the Grothendieck-Néron-Ogg-Shafarevic formula, we have then

$$
\begin{aligned}
\operatorname{deg}\left(M_{k}(f, T)\right) & =\operatorname{Swan}_{\infty}\left(\operatorname{Sym}^{k} \mathrm{Ai}_{f}\right)-\operatorname{rank}\left(\operatorname{Sym}^{k} \mathrm{Ai}_{f}\right) \\
& =\operatorname{Swan}_{\infty}\left(\operatorname{Sym}^{k} \mathrm{Ai}_{f}\right)-\left(\begin{array}{c}
k+d-2 \\
d-2
\end{array}\right) .
\end{aligned}
$$

In order to compute the Swan conductor of $\mathrm{Sym}^{k} \mathrm{Ai}_{f}$ we have to study the sheaf $\mathrm{Ai}_{f}$ as a representation of the inertia group $I_{\infty}$ of $\mathbb{A}_{\mathbb{F}_{q}}$ at infinity. Since $\mathcal{L}_{\psi(f)}$ is lisse on $\mathbb{A}^{1}$, as a representation of the decomposition group at infinity we have $\mathrm{Ai}_{f} \cong \mathcal{F}_{\infty, \infty}\left(\mathcal{L}_{\psi(f)}\right)$, where $\mathcal{F}_{\infty, \infty}$ is the local Fourier transform as defined in [22].

Recently, Fu [1] and, independently, Abbes and Saito 1] have given an explicit description of the different local Fourier transforms for a wide class of $\ell$-adic sheaves. We will mainly be using the description given in [1, which works over an arbitrary (not necessarily algebraically closed) perfect base field, and therefore gives an explicit formula for $\mathrm{Ai}_{f}$ as a representation of the decomposition group $D_{\infty}$.

If $S_{(\infty)}$ is the henselization of the local ring of $\mathbb{P}_{\mathbb{F}_{q}}^{1}$ at infinity with uniformizer $1 / t$, the triple $\left(\mathcal{L}_{\psi(f(t))}, t,-f^{\prime}(t)\right)$ is a Legendre triple in the sense of [1, Definition 2.16]. Therefore by [1, Theorem 3.9] we conclude that, as a representation of $D_{\infty}, \mathrm{Ai}_{f}$ is isomorphic to

$$
\left(-f^{\prime}\right)_{\star}\left(\mathcal{L}_{\psi(f(t))} \otimes \mathcal{L}_{\psi\left(-t f^{\prime}(t)\right)} \otimes \mathcal{L}_{\rho\left(\frac{1}{2} f^{\prime \prime}(t)\right)} \otimes \mathcal{Q}\right)=\left(-f^{\prime}\right)_{\star}\left(\mathcal{L}_{\psi\left(f(t)-t f^{\prime}(t)\right)} \otimes \mathcal{L}_{\rho\left(\frac{1}{2} f^{\prime \prime}(t)\right)} \otimes \mathcal{Q}\right)
$$

where $\rho$ is the unique character $I_{\infty} \rightarrow \overline{\mathbb{Q}}_{\ell}^{\star}$ of order $2, \mathcal{L}_{\rho}$ the corresponding Kummer sheaf and $\mathcal{Q}$ is the pull-back of the character $\operatorname{Gal}\left(\overline{\mathbb{F}}_{q} / \mathbb{F}_{q}\right) \rightarrow \overline{\mathbb{Q}}_{\ell}$ mapping the geometric Frobenius to the quadratic Gauss sum $g(\psi, \rho):=$ $-\sum_{t \in \mathbb{F}_{q}^{\star}} \psi(t) \rho(t)$.

Write $f(t)=\sum_{i=0}^{d} c_{i} t^{i}$. For simplicity, from now on we will assume that $\mathbb{F}_{q}$ contains the $2(d-1)$-th roots of $-d c_{d}$ (which can always be achieved by a finite extension of the base field). Following [11, Proposition 3.1] we can find an invertible power series $\sum_{i \geq 0} r_{i} t^{-i} \in \mathbb{F}_{q}\left[\left[t^{-1}\right]\right]$ with $r_{0}^{d-1}=-d c_{d}$ such that $u(t):=t \sum_{i \geq 0} r_{i} t^{-i}$ is a solution to $f^{\prime}(t)+u(t)^{d-1}=0$ (the other solutions being $\zeta u(t)$ for every $(d-1)$-th root of unity $\zeta$ ). The map $\phi: 1 / t \mapsto 1 / u(t)$ defines an automorphism $S_{(\infty)} \rightarrow S_{(\infty)}$, and by construction $-f^{\prime}=[d-1] \circ \phi$, where $[d-1]$ is the $(d-1)$-th power map. $\mathrm{So}_{\mathrm{Ai}}$ is isomorphic to

$$
\begin{aligned}
{[d-1]_{\star} \phi_{\star}\left(\mathcal{L}_{\psi\left(f(t)-t f^{\prime}(t)\right)} \otimes \mathcal{L}_{\rho\left(\frac{1}{2} f^{\prime \prime}(t)\right)} \otimes \mathcal{Q}\right) } & =[d-1]_{\star}\left(\phi^{-1}\right)^{\star}\left(\mathcal{L}_{\psi\left(f(t)-t f^{\prime}(t)\right)} \otimes \mathcal{L}_{\rho\left(\frac{1}{2} f^{\prime \prime}(t)\right)} \otimes \mathcal{Q}\right) \\
& =[d-1]_{\star}\left(\mathcal{L}_{\psi\left(f(v(t))+v(t) t^{d-1}\right)} \otimes \mathcal{L}_{\rho\left(\frac{1}{2} f^{\prime \prime}(v(t))\right)} \otimes \mathcal{Q}\right) \\
& =[d-1]_{\star}\left(\mathcal{L}_{\psi\left(f(v(t))+v(t) t^{d-1}\right)} \otimes \mathcal{L}_{\rho\left(\frac{1}{2} f^{\prime \prime}(v(t))\right)}\right) \otimes \mathcal{Q}
\end{aligned}
$$

since $[d-1]^{\star} \mathcal{Q}=\mathcal{Q}$, where $v(t):=\phi^{-1}(t)=t \sum_{i \geq 0} s_{i} t^{-i}$.

Let $g(t)$ be the polynomial of degree $d$ obtained from $f(v(t))+v(t) t^{d-1}$ by removing the terms with negative powers of $t$. It is important to notice that the coefficients of $g$ are polynomials in the coefficients of $f$. More precisely, if we write $g(t)=\sum b_{i} t^{i}$, the coefficient $b_{i}$ is a polynomial in the coeficients $a_{i}, a_{i+1}, \ldots, a_{d}$ of $f$. Since $\mathcal{L}_{\psi(h(t))}$ is trivial as a representation of $D_{\infty}$ for any $h(t) \in t^{-1} \mathbb{F}_{q}\left[\left[t^{-1}\right]\right]$, we have an isomorphism $\mathcal{L}_{\psi\left(f(v(t))+v(t) t^{d-1}\right)} \cong \mathcal{L}_{\psi(g(t))}$ as representations of $D_{\infty}$.

On the other hand, from $f^{\prime}(v(t))+t^{d-1}=0$ we get $f^{\prime \prime}(v(t)) v^{\prime}(t)+(d-1) t^{d-2}=0$, so $\mathcal{L}_{\rho\left(\frac{1}{2} f^{\prime \prime}(v(t))\right)}=$ $\mathcal{L}_{\rho\left(-\frac{d-1}{2} v^{\prime}(t) t^{d-2}\right)}$. Since $v^{\prime}(t)=\sum_{i \geq 0}(1-i) s_{i} t^{-i}=s_{0}\left(1+\sum_{i \geq 2}(1-i) \frac{s_{i}}{s_{0}} t^{-i}\right)$ and $1+\sum_{i \geq 2}(1-i) \frac{s_{i}}{s_{0}} t^{-i}$ is a square in $\mathbb{F}_{q}\left[\left[t^{-1}\right]\right]$, we have $\mathcal{L}_{\rho\left(-\frac{d-1}{2} v^{\prime}(t) t^{d-2}\right)}=\mathcal{L}_{\rho\left(-\frac{d-1}{2} s_{0} t^{d-2}\right)}=\mathcal{L}_{\rho\left(\frac{d(d-1)}{2} c_{d}\left(s_{0} t\right)^{d-2}\right)}\left(\right.$ since $\left.s_{0}^{d-1}=-1 / d c_{d}\right)$. So we finally get

$$
\mathrm{Ai}_{f} \cong[d-1]_{\star}\left(\mathcal{L}_{\psi(g(t))} \otimes \mathcal{L}_{\rho^{d}\left(s_{0} t\right)}\right) \otimes \mathcal{L}_{\rho\left(d(d-1) c_{d} / 2\right)} \otimes \mathcal{Q}
$$

We can now easily compute the Swan conductor at infinity of its symmetric powers. By [19, 1.13.1],

$$
\operatorname{Swan}_{\infty} \operatorname{Sym}^{k} \operatorname{Ai}_{f}=\frac{1}{d-1} \operatorname{Swan}_{\infty}[d-1]^{\star} \operatorname{Sym}^{k} \operatorname{Ai}_{f}=\frac{1}{d-1} \operatorname{Swan}_{\infty} \operatorname{Sym}^{k}[d-1]^{\star} \operatorname{Ai}_{f}
$$

Lemma 3.1. Let $\zeta$ be a primitive $(d-1)$-th root of unity if $\mathbb{F}_{q}, I_{\infty}^{d-1}$ the unique closed subgroup of $I_{\infty}$ of index $d-1$. As a representation of $I_{\infty}^{d-1}$, the restriction $[d-1]^{\star} \mathrm{Ai}_{f}$ of $\mathrm{Ai}_{f}$ is isomorphic to the direct sum

$$
\bigoplus_{i=0}^{d-2} \mathcal{L}_{\psi\left(g\left(\zeta^{i} t\right)\right)} \otimes \mathcal{L}_{\rho^{d}\left(s_{0} \zeta^{i} t\right)} \cong \bigoplus_{i=0}^{d-2} \mathcal{L}_{\psi\left(g\left(\zeta^{i} t\right)\right)} \otimes \mathcal{L}_{\rho^{d}(t)}
$$


Proof. Since $\left(\zeta^{i}\right)^{\star} \mathcal{L}_{\psi(g)}=\mathcal{L}_{\psi\left(g\left(\zeta^{i} t\right)\right)},\left(\zeta^{i}\right)^{\star} \mathcal{L}_{\rho^{d}\left(s_{0} t\right)}=\mathcal{L}_{\rho^{d}\left(s_{0} \zeta^{i} t\right)}$ and $[d-1] \circ \zeta^{i}=[d-1]$ for every $i$, we have $[d-1]_{\star}\left(\mathcal{L}_{\psi\left(g\left(\zeta^{i} t\right)\right)} \otimes \mathcal{L}_{\rho^{d}\left(s_{0} \zeta^{i} t\right)}\right)=[d-1]_{\star}\left(\mathcal{L}_{\psi(g(t))} \otimes \mathcal{L}_{\rho^{d}\left(s_{0} t\right)}\right)$, and therefore by Frobenius reciprocity Hom I $_{\infty}-1([d-$ $\left.1]^{\star} \mathrm{Ai}_{f}, \mathcal{L}_{\psi\left(g\left(\zeta^{i} t\right)\right)} \otimes \mathcal{L}_{\rho^{d}\left(s_{0} \zeta^{i} t\right)}\right)=\operatorname{Hom}_{I_{\infty}}\left(\mathrm{Ai}_{f},[d-1]_{\star}\left(\mathcal{L}_{\psi\left(g\left(\zeta^{i} t\right)\right)} \otimes \mathcal{L}_{\rho^{d}\left(s_{0} \zeta^{i} t\right)}\right)\right)=\operatorname{Hom}_{I_{\infty}}\left(\mathrm{Ai}_{f}, \mathrm{Ai}_{f}\right) \cong \mathbb{\mathbb { Q }}_{\ell}$ since the latter is an irreducible representation of $I_{\infty}$. So for every $i, \mathcal{L}_{\psi\left(g\left(\zeta^{i} t\right)\right)} \otimes \mathcal{L}_{\rho^{d}\left(s_{0} \zeta^{i} t\right)}$ is a subrepresentation of $[d-1]^{\star} \mathrm{Ai}_{f}$.

Now $\mathcal{L}_{\psi\left(g\left(\zeta^{i} t\right)\right)} \otimes \mathcal{L}_{\rho^{d}\left(s_{0} \zeta^{i} t\right)}$ and $\mathcal{L}_{\psi\left(g\left(\zeta^{j} t\right)\right)} \otimes \mathcal{L}_{\rho^{d}\left(s_{0} \zeta^{j} t\right)}$ are isomorphic if and only if $\mathcal{L}_{\psi\left(g\left(\zeta^{i} t\right)\right)}$ and $\mathcal{L}_{\psi\left(g\left(\zeta^{j} t\right)\right)}$ are, if and only if $g\left(\zeta^{i} t\right)-g\left(\zeta^{j} t\right)=h^{p}-h$ for some $h \in \overline{\mathbb{F}}_{q}[t]$. Since $p>d$, this can only happen if $g\left(\zeta^{i} t\right)=g\left(\zeta^{j} t\right)$. Comparing the highest degree coefficients we conclude that $\zeta^{i}$ and $\zeta^{j}$ must be equal. Therefore the direct sum of the $\mathcal{L}_{\psi\left(g\left(\zeta^{i} t\right)\right)} \otimes \mathcal{L}_{\rho^{d}\left(s_{0} \zeta^{i} t\right)}$ for $i=0, \ldots, d-2$ injects into $[d-1]^{\star} \mathrm{Ai}_{f}$ and we conclude that it must be isomorphic to it, since they have the same rank.

Consequently, we have an isomorphism of $\overline{\mathbb{Q}}_{\ell}\left[I_{\infty}\right]$-modules

$$
\operatorname{Sym}^{k}[d-1]^{\star} \mathrm{Ai}_{f} \cong \bigoplus_{a_{0}+a_{1}+\cdots+a_{d-2}=k} \mathcal{L}_{\psi\left(\sum_{i=0}^{d-2} a_{i} g\left(\zeta^{i} t\right)\right)} \otimes \mathcal{L}_{\rho^{d k}(t)} .
$$

For every finite subset $I \subset \mathbb{Z}$ and every integer $k \geq 0$ define

$$
S_{d-1}(k, I):=\left\{\left(a_{0}, \ldots, a_{d-2}\right) \in \mathbb{Z}_{\geq 0}^{d-1} \mid a_{0}+a_{1}+\cdots+a_{d-2}=k, a_{0}+a_{1} \zeta^{i}+\cdots+a_{d-2} \zeta^{i(d-2)}=0 \text { for every } i \in I\right\}
$$

It is clear from the definition that $S_{d-1}(k, I)=S_{d-1}\left(k, I^{\prime}\right)$ if $\phi(I)=\phi\left(I^{\prime}\right)$, where $\phi: \mathbb{Z} \rightarrow \mathbb{Z} /(d-1) \mathbb{Z}$ is reduction modulo $d-1$. Also, $S_{d-1}(k, I)=\emptyset$ if $p$ does not divide $k$ and $I \cap(d-1) \mathbb{Z} \neq \emptyset$. The number of elements in $S_{d-1}(k, I)$ can be conveniently expressed in terms of a generating function:

Lemma 3.2. Let $F_{d-1}(I ; T):=\sum_{k=0}^{\infty} \# S_{d-1}(k, I) T^{k}$. Then

$$
F_{d-1}(I ; T)=\frac{1}{q^{\# I}} \sum_{\gamma \in\left(\mathbb{F}_{q}\right)^{I}} \prod_{j=0}^{d-2}\left(1-\psi\left(\sum_{i \in I} \gamma_{i} \zeta^{j i}\right) T\right)^{-1}
$$

where $\psi$ is any non-trivial additive character of $\mathbb{F}_{q}$.

Proof. From the definition,

$$
F_{d-1}(I ; T)=\sum_{\left(a_{0}, \ldots, a_{d-2}\right) \in \mathbb{Z}_{\geq 0}^{d-1}} \prod_{i \in I} \delta\left(a_{0}+a_{1} \zeta^{i}+\cdots+a_{d-2} \zeta^{i(d-2)}\right) T^{a_{0}+a_{1}+\cdots+a_{d-2}}
$$

where $\delta(a)=1$ if $a=0,0$ otherwise. Equivalently, $\delta(a)=\frac{1}{q} \sum_{\gamma \in \mathbb{F}_{q}} \psi(\gamma a)$. So we get

$$
\begin{aligned}
F_{d-1}(I ; T) & =\sum_{\left(a_{0}, \ldots, a_{d-2}\right) \in \mathbb{Z}_{\geq 0}^{d-1}} \prod_{i \in I} \frac{1}{q} \sum_{\gamma_{i} \in \mathbb{F}_{q}} \psi\left(\gamma_{i}\left(a_{0}+a_{1} \zeta^{i}+\cdots+a_{d-2} \zeta^{i(d-2)}\right)\right) T^{a_{0}+a_{1}+\cdots+a_{d-2}} \\
& =\sum_{\left(a_{0}, \ldots, a_{d-2}\right) \in \mathbb{Z}_{\geq 0}^{d-1}} \sum_{\gamma \in\left(\mathbb{F}_{q}\right)^{I}} \frac{1}{q^{\# I}}\left(\prod_{i \in I} \psi\left(\gamma_{i} a_{0}\right)\right) T^{a_{0}}\left(\prod_{i \in I} \psi\left(\gamma_{i} a_{1} \zeta^{i}\right)\right) T^{a_{1}} \cdots\left(\prod_{i \in I} \psi\left(\gamma_{i} a_{d-2} \zeta^{(d-2) i}\right)\right) T^{a_{d-2}} \\
& =\frac{1}{q^{\# I}} \sum_{\gamma \in\left(\mathbb{F}_{q}\right)^{I}} \sum_{\left(a_{0}, \ldots, a_{d-2}\right) \in \mathbb{Z}_{\geq 0}^{d-1}} \psi\left(\sum_{i \in I} \gamma_{i}\right)^{a_{0}} T^{a_{0}} \psi\left(\sum_{i \in I} \gamma_{i} \zeta^{i}\right)^{a_{1}} T^{a_{1}} \cdots \psi\left(\sum_{i \in I} \gamma_{i} \zeta^{(d-2) i}\right)^{a_{d-2}} T^{a_{d-2}} \\
& =\frac{1}{q^{\# I}} \sum_{\gamma \in\left(\mathbb{F}_{q}\right)^{I}}\left(\sum_{a_{0} \in \mathbb{Z}_{\geq 0}} \psi\left(\sum_{i \in I} \gamma_{i}\right)^{a_{0}} T^{a_{0}}\right)\left(\sum_{a_{1} \in \mathbb{Z}_{\geq 0}} \psi\left(\sum_{i \in I} \gamma_{i} \zeta^{i}\right)^{a_{1}} T^{a_{1}}\right) \cdots\left(\sum_{a_{d-2} \in \mathbb{Z}_{\geq 0}} \psi\left(\sum_{i \in I} \gamma_{i} \zeta^{(d-2) i}\right)^{a_{d-2}} T^{a_{d-2}}\right) \\
& =\frac{1}{q^{\# I}} \sum_{\gamma \in\left(\mathbb{F}_{q}\right)^{I}} \prod_{j=0}^{d-2}\left(1-\psi\left(\sum_{i \in I} \gamma_{i} \zeta^{j i}\right) T\right)^{-1} .
\end{aligned}
$$

Write $g(t)=\sum_{j=0}^{d} b_{j} t^{j}$, and let $J=\left\{1 \leq j \leq d \mid b_{j} \neq 0\right\}$ and $J_{\geq j}:=J \cap\{j, j+1, \ldots, d\}$ for every $j \in$ $\{1, \ldots, d, d+1\}$. We have

$$
\begin{aligned}
\operatorname{Swan}_{\infty} \operatorname{Sym}^{k}[d-1]^{\star} \mathrm{Ai}_{f} & =\sum_{a_{0}+a_{1}+\cdots+a_{d-2}=k} \operatorname{Swan}_{\infty} \mathcal{L}_{\psi\left(\sum_{i=0}^{d-2} a_{i} g\left(\zeta^{i} t\right)\right)} \otimes \mathcal{L}_{\rho^{d k}(t)} \\
& =\sum_{a_{0}+a_{1}+\cdots+a_{d-2}=k} \operatorname{deg}\left(\sum_{i=0}^{d-2} a_{i} g\left(\zeta^{i} t\right)\right)
\end{aligned}
$$


and

$$
\sum_{i=0}^{d-2} a_{i} g\left(\zeta^{i} t\right)=\sum_{i=0}^{d-2} a_{i} \sum_{j=0}^{d} b_{j} \zeta^{i j} t^{j}=\sum_{j=0}^{d}\left(b_{j} \sum_{i=0}^{d-2} \zeta^{i j}\right) t^{j}
$$

so its degree is the greatest $j$ such that $b_{j} \sum_{i=0}^{d-2} \zeta^{i j} \neq 0$. Therefore we get

$$
\begin{aligned}
(d-1) \operatorname{Swan}_{\infty} \operatorname{Sym}^{k} \operatorname{Ai}_{f} & =\operatorname{Swan}_{\infty} \operatorname{Sym}^{k}[d-1]^{\star} \operatorname{Ai}_{f} \\
& =\sum_{j \in J} j \cdot\left(\# S_{d-1}\left(k, J_{\geq j+1}\right)-\# S_{d-1}\left(k, J_{\geq j}\right)\right) \\
& =d\left(\begin{array}{c}
k+d-2 \\
d-2
\end{array}\right)-\sum_{j \in J} h(j) \cdot \# S_{d-1}\left(k, J_{\geq j}\right)
\end{aligned}
$$

where $h(j):=j-\sup \left(J-J_{\geq j}\right)$ is the "gap" between the $t^{j}$ term and the next lower degree term in $g(t)$. Taking the corresponding generating function we get the formula

Corollary 3.3. Let $G(f ; T):=\sum_{k=0}^{\infty}\left(\operatorname{Swan}_{\infty} \operatorname{Sym}^{k} \mathrm{Ai}_{f}\right) T^{k}$, then

$$
G(f ; T)=\frac{d}{(d-1)(1-T)^{d-1}}-\frac{1}{d-1} \sum_{j \in J} h(j) \cdot F_{d-1}\left(J_{\geq j} ; T\right)
$$

Using the previous formula for the degree, we deduce

Corollary 3.4. The degree of $M_{k}(f ; T)$ is the $k$-th coefficient of the power series expansion of

$$
\frac{1}{(d-1)(1-T)^{d-1}}-\frac{1}{d-1} \sum_{j \in J} h(j) \cdot F_{d-1}\left(J_{\geq j} ; T\right) .
$$

Corollary 3.5. For every $J \subset\{1, \ldots, d-1\}$, let $\mathcal{P}_{d}(J)$ be the subspace of the affine space $\mathcal{P}_{d}$ of polynomials of degree $d$ over $k$ such that $b_{j}=0$ if and only if $j \in J$. The sets $\left\{\mathcal{P}_{d}(J) \mid J \subseteq\{1, \ldots, d-1\}\right\}$ define a stratification of $\mathcal{P}_{d}$ such that the degree of $M_{k}(f ; T)$ is constant in each stratum.

\section{The trivial factor}

Suppose $p>d$ and the monodromy of $\mathrm{Ai}_{f}$ is not finite. We will now study the weights of the (reciprocal) roots of the polynomial $M_{k}(f, T)$. Let us first consider the easier case where $d$ is even, and therefore $\mathrm{Ai}_{f}$ is isomorphic to $[d-1]_{\star} \mathcal{L}_{\psi(g(t))} \otimes \mathcal{L}_{\rho\left(d(d-1) c_{d} / 2\right)} \otimes \mathcal{Q}$ as a representation of $D_{\infty}$. Let $D_{\infty}^{d-1}=\operatorname{Gal}\left(\overline{\mathbb{F}_{q}((1 / t))} / \mathbb{F}_{q}\left(\left(1 / t^{1 /(d-1)}\right)\right)\right)$, denote by $\alpha: D_{\infty}^{d-1} \rightarrow \overline{\mathbb{Q}}_{\ell}^{\star}$ the character corresponding to the sheaf $\mathcal{L}_{\psi(g)}$, and let $b \in I_{\infty}$ be a generator of the cyclic group $D_{\infty} / D_{\infty}^{d-1} \cong I_{\infty} / I_{\infty}^{d-1}$. By the explicit description of induced representations, there is a basis $\left\{v_{0}, \ldots, v_{d-2}\right\}$ of the underlying vector space $V$ such that $a \cdot v_{0}=\alpha(a) v_{0}$ for every $a \in I_{\infty}^{d-1}$ and $b \cdot v_{i}=v_{i+1}$ for $i=0, \ldots, d-3$. Then $b \cdot v_{d-2}=b^{d-1} \cdot v_{0}=\alpha\left(b^{d-1}\right) v_{0}$. Replacing $b$ by $a^{-1} b$, where $a \in I_{\infty}^{d-1}$ is an element such that $\alpha(a)^{d-1}=\alpha\left(b^{d-1}\right.$ ) (which is always possible since the values of $\alpha$ are the $p$-th roots of unity and $d-1$ is prime to $p$ since $p>d$ ) we may assume without loss of generality that $\alpha\left(b^{d-1}\right)=1$.

Furthermore, for any $a \in I_{\infty}^{d-1}$ we have $a \cdot v_{i}=\left(a b^{i}\right) \cdot v_{0}=\left(b^{i} b^{-i} a b^{i}\right) \cdot v_{0}=b^{i} \cdot \alpha\left(b^{-i} a b^{i}\right) v_{0}=\alpha\left(b^{-i} a b^{i}\right) v_{i}$. So the restriction of $\mathrm{Ai}_{f}$ to $D_{\infty}^{d-1}$ is the direct sum of the characters $a \mapsto \alpha_{i}(a):=\alpha\left(b^{-i} a b^{i}\right)$. But we already know that it is the direct sum of the characters associated to the sheaves $\mathcal{L}_{\psi\left(g\left(\zeta^{i} t\right)\right)} \otimes \mathcal{L}_{\rho\left(d(d-1) c_{d} / 2\right)} \otimes \mathcal{Q}$, so these two sets of characters are identical. Replacing $b$ by a suitable power of itself we may assume that $\alpha_{i}$ is the character associated to $\mathcal{L}_{\psi\left(g\left(\zeta^{i} t\right)\right)} \otimes \mathcal{L}_{\rho\left(d(d-1) c_{d} / 2\right)} \otimes \mathcal{Q}$. In particular, $\prod_{i=0}^{d-2} \alpha_{i}^{a_{i}}$ is geometricaly trivial (that is, trivial on $\left.I_{\infty}^{d-1}\right)$ if and only if $\sum a_{i} g\left(\zeta^{i} t\right)$ is a constant in $\mathbb{F}_{q}[t]$, that is, if and only if $\sum a_{i} \zeta^{i j}=0$ for every $j \in J$.

We turn now to the case $d$ odd. Let $\chi$ be a multiplicative character of $\mathbb{F}_{q}$ of order $2(d-1)$ (which exists, since we are assuming that $\mathbb{F}_{q}$ contains the $2(d-1)$-th roots of unity). Then by the projection formula $\mathrm{Ai}_{f}$ is isomorphic to $[d-1]_{\star}\left(\mathcal{L}_{\psi(g(t))} \otimes \mathcal{L}_{\rho\left(s_{0} t\right)}\right) \otimes \mathcal{L}_{\rho\left(d(d-1) c_{d} / 2\right)} \otimes \mathcal{Q} \cong\left([d-1]_{\star} \mathcal{L}_{\psi(g(t))}\right) \otimes \mathcal{L}_{\chi\left(s_{0} t\right)} \otimes \mathcal{L}_{\rho\left(d(d-1) c_{d} / 2\right)} \otimes \mathcal{Q}$. Let $\alpha_{i}: D_{\infty}^{d-1} \rightarrow \overline{\mathbb{Q}}_{\ell}^{\star}$ (respectively $\beta: D_{\infty} \rightarrow \overline{\mathbb{Q}}_{\ell}^{\star}$ ) be the character corresponding to the sheaf $\mathcal{L}_{\psi\left(g\left(\zeta^{i} t\right)\right)}$ (resp. $\left.\mathcal{L}_{\chi\left(s_{0} t\right)}\right)$. Proceeding as in the $d$ even case, we find a generator $b \in I_{\infty}$ of $D_{\infty} / D_{\infty}^{d-1}$ and a basis $\left\{v_{0}, \ldots, v_{d-2}\right\}$ of $V$ such that $a \cdot v_{i}=\alpha_{i}(a) \beta(a) v_{i}$ for $a \in D_{\infty}^{d-1}$ and $b \cdot v_{i}=\beta(b) v_{i+1}$ for $i=0, \ldots, d-3, b \cdot v_{d-2}=\beta(b) v_{0}$. In this case, $\prod_{i=0}^{d-2} \alpha_{i}^{a_{i}} \beta^{a_{i}}$ is trivial on $I_{\infty}^{d-1}$ if and only if $\sum a_{i} g\left(\zeta^{i} t\right)$ is a constant in $\mathbb{F}_{q}[t]$ and $\sum a_{i}$ is even (since $\alpha_{i}$ has order $p$ and $\beta$ restricted to $I_{\infty}^{d-1}$ has order 2). 
We can now compute the dimension of the invariant subspace of the action of $I_{\infty}$ on $\mathrm{Sym}^{k} \mathrm{Ai}_{f}$, in very much the same way it is done for the Kloosterman sheaf in [12, Lemma 2.1]. Its underlying vector space is $\operatorname{Sym}^{k} V$. An element $w$ is given by a linear combination

$$
w=\sum_{a_{0}+\cdots+a_{d-2}=k} c_{a_{0} \cdots a_{d-2}} v_{0}^{a_{0}} \cdots v_{d-2}^{a_{d-2}} .
$$

In the $d$ even case we have

$$
a \cdot \sum_{a_{0}+\cdots+a_{d-2}=k} c_{a_{0} \cdots a_{d-2}} v_{0}^{a_{0}} \cdots v_{d-2}^{a_{d-2}}=\sum_{a_{0}+\cdots+a_{d-2}=k} c_{a_{0} \cdots a_{d-2}}\left(\alpha_{0}^{a_{0}} \cdots \alpha_{d-2}^{a_{d-2}}\right)(a) v_{0}^{a_{0}} \cdots v_{d-2}^{a_{d-2}}
$$

for $a \in I_{\infty}^{d-1}$ and

$$
b \cdot \sum_{a_{0}+\cdots+a_{d-2}=k} c_{a_{0} \cdots a_{d-2}} v_{0}^{a_{0}} \cdots v_{d-2}^{a_{d-2}}=\sum_{a_{0}+\cdots+a_{d-2}=k} c_{a_{0} \cdots a_{d-2}} v_{1}^{a_{0}} v_{2}^{a_{1}} \cdots v_{0}^{a_{d-2}} .
$$

So $w$ is fixed by $I_{\infty}$ if and only if the character $\alpha_{0}^{a_{0}} \cdots \alpha_{d-2}^{a_{d-2}}$ is trivial whenever $c_{a_{0} \cdots a_{d-2}} \neq 0$ and $c_{a_{0} \cdots a_{d-2}}=$ $c_{a_{d-2} a_{0} \cdots a_{d-3}}$ for all $a_{0}, \ldots, a_{d-2}$. A basis for the invariant subspace is thus given by all distinct sums of the form (setting $v_{d-1+l}:=v_{l}$ for all $l \geq 0$ ):

$$
\sum_{j=0}^{d-2} v_{j}^{a_{0}} v_{j+1}^{a_{1}} \cdots v_{j+d-2}^{a_{d-2}}
$$

for all $a_{0}, \ldots, a_{d-2}$ such that $\alpha_{0}^{a_{0}} \cdots \alpha_{d-2}^{a_{d-2}}$ is trivial, that is, such that $\sum a_{i} \zeta^{i j}=0$ in $\mathbb{F}_{q}$ for every $j \in J$.

In the $d$ odd case we get

$$
g \cdot \sum_{a_{0}+\cdots+a_{d-2}=k} c_{a_{0} \cdots a_{d-2}} v_{0}^{a_{0}} \cdots v_{d-2}^{a_{d-2}}=\sum_{a_{0}+\cdots+a_{d-2}=k} c_{a_{0} \cdots a_{d-2}}\left(\alpha_{0}^{a_{0}} \cdots \alpha_{d-2}^{a_{d-2}}\right)(g) \beta^{k}(g) v_{0}^{a_{0}} \cdots v_{d-2}^{a_{d-2}}
$$

for $g \in I_{\infty}^{d-1}$ and

$$
h \cdot \sum_{a_{0}+\cdots+a_{d-2}=k} c_{a_{0} \cdots a_{d-2}} v_{0}^{a_{0}} \cdots v_{d-2}^{a_{d-2}}=\sum_{a_{0}+\cdots+a_{d-2}=k} c_{a_{0} \cdots a_{d-2}} \beta(h)^{k} v_{1}^{a_{0}} v_{2}^{a_{1}} \cdots v_{0}^{a_{d-2}} .
$$

So $w$ is fixed by $I_{\infty}$ if and only if the character $\alpha_{0}^{a_{0}} \cdots \alpha_{d-2}^{a_{d-2}} \beta^{k}$ of $I_{\infty}^{d-1}$ is trivial whenever $c_{a_{0} \cdots a_{d-2}} \neq 0$ and $c_{a_{0} \cdots a_{d-2}}=c_{a_{d-2} a_{0} \cdots a_{d-3}} \beta(h)^{k}$ for all $a_{0}, \ldots, a_{d-2}$. Since all $\alpha_{i}$ 's have order $p$ and the restriction of $\beta$ to $I_{\infty}^{d-1}$ has order $2, \alpha_{0}^{a_{0}} \cdots \alpha_{d-2}^{a_{d-2}} \beta^{k}$ is trivial if and only if both $\alpha_{0}^{a_{0}} \cdots \alpha_{d-2}^{a_{d-2}}$ and $\beta^{k}$ are trivial as characters of $I_{\infty}^{d-1}$, that is, if and only if $\sum a_{i} \zeta^{i j}=0$ in $\mathbb{F}_{q}$ for every $j \in J$ and $k$ is even. In particular, there are no non-zero invariants for $I_{\infty}$ if $k$ is odd. If $k$ is even, a generating set for the invariant subspace is given by all distinct sums of the form

$$
\sum_{j=0}^{d-2} \beta(h)^{j k} v_{j}^{a_{0}} v_{j+1}^{a_{1}} \cdots v_{j+d-2}^{a_{d-2}}
$$

for all $a_{0}, \ldots, a_{d-2}$ such that $\sum a_{i} \zeta^{i j}=0$ in $\mathbb{F}_{q}$ for every $j \in J$. Let $r$ be the size of the orbit of $\left(a_{0}, \ldots, a_{d-2}\right)$ under the action of $\mathbb{Z} /(d-1) \mathbb{Z}$ by cyclic permutations. If $r \neq d-1$, we can write

$$
\sum_{j=0}^{d-2} \beta(h)^{j k} v_{j}^{a_{0}} v_{j+1}^{a_{1}} \cdots v_{j+d-2}^{a_{d-2}}=\sum_{j=0}^{r-1} \beta(h)^{j k}\left(1+\beta(h)^{r k}+\cdots+\beta(h)^{\left(\frac{d-1}{r}-1\right) r k}\right) v_{j}^{a_{0}} v_{j+1}^{a_{1}} \cdots v_{j+d-2}^{a_{d-2}} .
$$

Notice that $k$ must be a multiple of $\frac{d-1}{r}$, since $k=\sum_{i=0}^{d-2} a_{i}=\frac{d-1}{r} \sum_{i=0}^{r-1} a_{i}$. If $\frac{r k}{d-1}$ is odd we have

$$
1+\beta(h)^{r k}+\cdots+\beta(h)^{\left(\frac{d-1}{r}-1\right) r k}=\frac{1-\beta(h)^{(d-1) k}}{1-\beta(h)^{r k}}=0,
$$

so the above sum vanishes. On the other hand, if $\frac{r k}{d-1}$ is even it is clear that the element

$$
\sum_{j=0}^{d-2} \beta(h)^{j k} v_{j}^{a_{0}} v_{j+1}^{a_{1}} \cdots v_{j+d-2}^{a_{d-2}}=\frac{d-1}{r} \sum_{j=0}^{r-1} \beta(h)^{j k} v_{j}^{a_{0}} v_{j+1}^{a_{1}} \cdots v_{j+d-2}^{a_{d-2}}
$$

is non-zero, and to different orbits correspond different elements. To summarize, we have 
Proposition 4.1. Let $T_{d-1}(k, J)$ be the set of orbits of the action of $\mathbb{Z} /(d-1) \mathbb{Z}$ on the set $S_{d-1}(k, J)$ by cyclic permutations, and let $U_{d-1}(k, J)$ be the subset of orbits such that $\frac{r k}{d-1}$ is even, where $r$ is their cardinality. If $d$ is even, the invariant subspace of the representation $\operatorname{Sym}^{k} \mathrm{Ai}_{f}$ of $I_{\infty}$ has dimension $\# T_{d-1}(k, J)$. If $d$ is odd and $k$ is even, it has dimension $\# U_{d-1}(k, J)$. If $d$ and $k$ are odd, the representation has no non-zero invariants.

The sequences $\# T_{d-1}(k, J)$ and $\# U_{d-1}(k, J)$ can also be described by means of generating functions. By Burnside's lemma, the dimension of the invariant subspace for $d$ even is given by

$$
\# T_{d-1}(k, J)=\frac{1}{d-1} \sum_{r=1}^{d-1} \#\left\{\left(a_{0}, a_{1}, \ldots, a_{d-2}\right) \mid a_{i}=a_{i+r} \bmod { }_{d-1}\right\}=\frac{1}{d-1} \sum_{r \mid d-1} \phi\left(\frac{d-1}{r}\right) \# S_{r}\left(\frac{k r}{d-1}, J\right)
$$

where $S_{r}(k, J)=\emptyset$ if $k$ is not an integer and $\phi$ is Euler's totient function. So the generating function for the sequence $\left\{\# T_{d-1}(k, J) \mid k \geq 0\right\}$ is

$$
\begin{aligned}
G_{d-1}(J ; T): & =\sum_{k=0}^{\infty} \# T_{d-1}(k, J) T^{k} \\
& =\sum_{k=0}^{\infty} \frac{1}{d-1} T^{k} \sum_{r \mid d-1} \phi\left(\frac{d-1}{r}\right) \# S_{r}\left(\frac{k r}{d-1}, J\right) \\
& =\frac{1}{d-1} \sum_{r \mid d-1} \phi\left(\frac{d-1}{r}\right) \sum_{\frac{d-1}{r} \mid k} \# S_{r}\left(\frac{k r}{d-1}, J\right) T^{k} \\
& =\frac{1}{d-1} \sum_{r \mid d-1} \phi\left(\frac{d-1}{r}\right) \sum_{s=0}^{\infty} \# S_{r}(s, J) T^{\frac{d-1}{r} s} \\
& =\frac{1}{d-1} \sum_{r \mid d-1} \phi\left(\frac{d-1}{r}\right) F_{r}\left(J ; T^{\frac{d-1}{r}}\right)
\end{aligned}
$$

Next, suppose that $d$ is odd, and let $\left(a_{0}, \ldots, a_{d-2}\right) \in S_{d-1}(k, J)$. Let $r$ be the number of elements in its orbit. Then $\sum_{i=0}^{r-1} a_{i}=\frac{k r}{d-1}$. We want to count the number of orbits such that this value is even. Since $k=\frac{k r}{d-1} \cdot \frac{d-1}{r}$, if the largest power of 2 that divides $d-1$ is smaller than the largest power of 2 dividing $k, \frac{k r}{d-1}$ must always be even. Suppose that the largest power of 2 that divides $k, 2^{\alpha(k)}$, divides $d-1$. Then $\frac{k r}{d-1}$ is odd if and only if $2^{\alpha(k)}$ divides $\frac{d-1}{r}$, if and only if $r$ divides $\frac{d-1}{2^{\alpha(k)}}$. Therefore $\# U_{d-1}(k, J)=\# T_{d-1}(k, J)$ if $2^{\alpha(k)}$ does not divide $d-1$ and $\# T_{d-1}(k, J)-\# T_{\frac{d-1}{2^{\alpha(k)}}}\left(\frac{k}{2^{\alpha(k)}}, J\right)$ if it does. The generating function is then

$$
\begin{aligned}
\sum_{k=0}^{\infty} \# U_{d-1}(k, J) T^{k} & =\sum_{k=0}^{\infty} \# T_{d-1}(k, J) T^{k}-\sum_{j \geq 1 ; 2^{j} \mid d-1} \sum_{l \text { odd }} \# T_{\frac{d-1}{2^{j}}}(l, J) T^{2^{j} l} \\
& =G_{d-1}(J ; T)-\sum_{j \geq 1 ; 2^{j} \mid d-1} H_{\frac{d-1}{2^{j}}}\left(J ; T^{2^{j}}\right)
\end{aligned}
$$

where

$$
H_{r}(J ; T):=\frac{1}{2}\left(G_{r}(J ; T)-G_{r}(J ;-T)\right) .
$$

Let $F \in D_{\infty}^{d-1} \subset D_{\infty}$ be a geometric Frobenius element, and $w=\sum_{j=0}^{d-2} v_{j}^{a_{0}} v_{j+1}^{a_{1}} \cdots v_{j+d-2}^{a_{d-2}}$ (resp. $w=$ $\left.\sum_{j=0}^{d-2} \beta(h)^{j k} v_{j}^{a_{0}} v_{j+1}^{a_{1}} \cdots v_{j+d-2}^{a_{d-2}}\right)$ a generator of the $I_{\infty}$-invariant subspace of $\operatorname{Sym}^{k} V . F$ acts on $v_{j}^{a_{0}} v_{j+1}^{a_{1}} \cdots v_{j+d-2}^{a_{d-2}}$ via the character corresponding to $\mathcal{L}_{\psi\left(\sum a_{i} g\left(\zeta^{j+i} t\right)\right)} \otimes \mathcal{L}_{\rho\left(d(d-1) c_{d} / 2\right)}^{\otimes k} \otimes \mathcal{Q}^{\otimes k}\left(\right.$ resp. $\mathcal{L}_{\psi\left(\sum a_{i} g\left(\zeta^{j+i} t\right)\right)} \otimes \mathcal{L}_{\rho\left(\Pi\left(s_{0} \zeta^{j+i} t\right)^{a_{i}}\right)} \otimes$ $\left.\mathcal{L}_{\rho\left(d(d-1) c_{d} / 2\right)}^{\otimes k} \otimes \mathcal{Q}^{\otimes k}\right)$. Since $\sum a_{i} g\left(\zeta^{j+i} t\right)$ must be a constant polynomial, we have $\mathcal{L}_{\psi\left(\sum a_{i} g\left(\zeta^{j+i} t\right)\right)} \cong \mathcal{L}_{\psi\left(k b_{0}\right)}$. Additionally, if $d$ is odd and $k$ even, $\mathcal{L}_{\rho\left(\Pi\left(s_{0} t\right)^{a_{i}}\right)}=\mathcal{L}_{\rho\left(s_{0} t\right)^{k}}$ is trivial. We conclude:

Proposition 4.2. A Frobenius geometric element at infinity acts on the $I_{\infty}$-invariant subspace of $\mathrm{Sym}^{k} \mathrm{Ai}_{f}$ by multiplication by $\psi\left(k b_{0}\right) \rho\left(d(d-1) c_{d} / 2\right)^{k} g(\psi, \rho)^{k}$.

As an immediate consequence we get

Corollary 4.3. The local L-function of $\operatorname{Sym}^{k} \mathrm{Ai}_{f}$ at infinity $\operatorname{det}\left(1-\operatorname{Frob} T \mid\left(\mathrm{Sym}^{k} \mathrm{Ai}_{f}\right)^{I_{\infty}}\right)$ is given by $(1-$ $\left.\psi\left(k b_{0}\right) \rho\left(d(d-1) c_{d} / 2\right)^{k} g(\psi, \rho)^{k} T\right)^{\# T_{d-1}(k, J)}$ if $d$ is even, $\left(1-\psi\left(k b_{0}\right) \rho\left(d(d-1) c_{d} / 2\right)^{k} g(\psi, \rho)^{k} T\right)^{\# U_{d-1}(k, J)}$ if $d$ is odd and $k$ is even, and 1 if $d$ and $k$ are odd. 
Theorem 4.4. The polynomial $M_{k}(f, T)$ decomposes as a product $P_{k}(f, T) Q_{k}(f, T)$, where $Q_{k}(f, T)$ is given by the formula in Corollary 4.3 and $P_{k}(d, T)$ satisfies a functional equation

$$
P(T)=c T^{r} \overline{P\left(1 / q^{k+1} T\right)}
$$

where $|c|=q^{r(k+1) / 2}$ and $r$ is its degree.

Proof. Let $j: \mathbb{A}^{1} \rightarrow \mathbb{P}^{1}$ be the inclusion. From the exact sequence

$$
0 \rightarrow \operatorname{Sym}^{k} \mathrm{Ai}_{f} \rightarrow j_{\star} \operatorname{Sym}^{k} \mathrm{Ai}_{f} \rightarrow\left(j_{\star} \operatorname{Sym}^{k} \mathrm{Ai}_{f}\right)_{\infty} \rightarrow 0
$$

we get an exact sequence of $\operatorname{Gal}\left(\overline{\mathbb{F}}_{q} / \mathbb{F}_{q}\right)$-modules

$$
0 \rightarrow\left(j_{\star} \operatorname{Sym}^{k} \mathrm{Ai}_{f}\right)^{I_{\infty}} \rightarrow \mathrm{H}_{c}^{1}\left(\mathbb{A}^{1}, \operatorname{Sym}^{k} \mathrm{Ai}_{f}\right) \rightarrow \mathrm{H}^{1}\left(\mathbb{P}^{1}, j_{\star} \operatorname{Sym}^{k} \mathrm{Ai}_{f}\right) \rightarrow 0
$$

and therefore a decomposition

$$
\begin{aligned}
M_{k}(f, T) & =\operatorname{det}\left(1-\operatorname{Frob} T \mid \mathrm{H}_{c}^{1}\left(\mathbb{A}^{1}, \operatorname{Sym}^{k} \mathrm{Ai}_{f}\right)\right) \\
& =\operatorname{det}\left(1-\operatorname{Frob} T \mid\left(j_{\star} \operatorname{Sym}^{k} \mathrm{Ai}_{f}\right)^{I_{\infty}}\right) \operatorname{det}\left(1-\operatorname{Frob} T \mid \mathrm{H}^{1}\left(\mathbb{P}^{1}, j_{\star} \operatorname{Sym}^{k} \mathrm{Ai}_{f}\right)\right) .
\end{aligned}
$$

The first factor is described by the previous corollary. On the other hand, by [8, Théorème 1.3] we have a perfect pairing

$$
\mathrm{H}^{1}\left(\mathbb{P}^{1}, j_{\star} \operatorname{Sym}^{k} \mathrm{Ai}_{f}\right) \times \mathrm{H}^{1}\left(\mathbb{P}^{1}, j_{\star} \operatorname{Sym}^{k} \widehat{\mathrm{Ai}_{f}}\right) \rightarrow \overline{\mathbb{Q}}_{\ell}(-k-1)
$$

where $\widehat{\mathrm{Ai}_{f}}$ is the dual of $\mathrm{Ai}_{f}$, which is constructed in the same way as $\mathrm{Ai}_{f}$ using the complex conjugate character $\bar{\psi}$ instead of $\psi$. If the eigenvalues of the action of Frobenius on $\mathrm{H}^{1}\left(\mathbb{P}^{1}, j_{\star} \mathrm{Sym}^{k} \mathrm{Ai}_{f}\right)$ are $\alpha_{1}, \cdots, \alpha_{r}$, so that $P_{k}(f, T)=\prod\left(1-\alpha_{i} T\right)$, it follows that $\overline{P_{k}(f, T)}=\prod\left(1-\left(q^{k+1} / \alpha_{i}\right) T\right)$ and therefore the functional equation holds. Applying the functional equation twice we get $|c|=q^{r(k+1) / 2}$.

\section{Some special cases}

We will now see how the previous results apply to some special values of $f$. First, consider the case $f(t)=t^{d}$. In this case the equation $f^{\prime}(t)+u(t)^{d-1}=0$ gives $u(t)=r_{0} t$, where $r_{0}^{d-1}=-d$. Then $v(t)=t / r_{0}$, and $g(t)=f(v(t))+v(t) t^{d-1}=t^{d}\left(1 / r_{0}^{d}+1 / r_{0}\right)=\frac{d-1}{d r_{0}} t^{d}$. By corollary 3.4 , we get that the degree of $M_{k}(f ; T)$ is the $k$-th coefficient in the power series expansion of

$$
\frac{1}{d-1}\left(\frac{1}{(1-T)^{d-1}}-d F_{d-1}(\{1\} ; T)\right)
$$

where

$$
F_{d-1}(\{1\} ; T)=\frac{1}{q} \sum_{\gamma \in \mathbb{F}_{q}} \prod_{j=0}^{d-2}\left(1-\psi\left(\gamma \zeta^{j}\right) T\right)^{-1}
$$

Explicitly,

$$
\operatorname{deg} M_{k}(f, T)=\frac{1}{d-1}\left(\left(\begin{array}{c}
k+d-2 \\
d-2
\end{array}\right)-d \cdot \# S_{d-1}(k,\{1\})\right) .
$$

In particular, for $d=3$

$$
F_{2}(\{1\} ; T)=\frac{1}{q} \sum_{\gamma \in \mathbb{F}_{q}}(1-\psi(\gamma) T)^{-1}(1-\psi(-\gamma) T)^{-1}=\frac{1}{p} \sum_{m=0}^{p-1}\left(1-\exp \left(\frac{2 \pi i m}{p}\right) T\right)^{-1}\left(1-\exp \left(\frac{-2 \pi i m}{p}\right) T\right)^{-1} .
$$

It is easily checked that $S_{2}(k,\{1\}):=\{(a, b) \mid a+b=k, a \equiv b(\bmod p)\}$ has $\left\lfloor\frac{k}{p}\right\rfloor+\delta$ elements, where $\delta=0$ (resp. $\delta=1)$ if $k-\left\lfloor\frac{k}{p}\right\rfloor$ is odd (resp. even). So in this case we get an explicit formula for the degree:

$$
\operatorname{deg} M_{k}\left(f(t)=t^{3} ; T\right)=\frac{1}{2}\left(k+1-3\left(\left\lfloor\frac{k}{p}\right\rfloor+\delta\right)\right)
$$

If $p>k$ this gives $(k+1) / 2$ for $k$ odd and $(k-2) / 2$ for $k$ even.

Corollary 4.3 states for $f(t)=t^{d}$ that the local $L$-function of $\operatorname{Sym}^{k} \mathrm{Ai}_{f}$ at infinity is $\left(1-\rho(d(d-1) / 2)^{k} g(\psi, \rho)^{k} T\right)^{\# T_{d-1}(k, J)}$ if $d$ is even, $\left(1-\rho(d(d-1) / 2)^{k} g(\psi, \rho)^{k} T\right)^{\# U_{d-1}(k, J)}$ if $d$ is odd and $k$ is even and 1 if $d$ and $k$ are odd. For $d=3$, we can again provide a more explicit expression. 
Since 3 is odd, the local $L$-function is 1 for $k$ odd. For $k$ even, we can write $\# S_{2}(k,\{1\})=\left\lfloor\frac{k}{p}\right\rfloor+\delta=2\left\lfloor\frac{k}{2 p}\right\rfloor+1$. Every orbit of $\mathbb{Z} / 2 \mathbb{Z}$ acting on $S_{2}(k,\{1\})$ has two elements except for $\{(k / 2, k / 2)\}$, so $\# T_{2}(k,\{1\})=\left\lfloor\frac{k}{2 p}\right\rfloor+1$. $U_{2}(k,\{1\})$ contains the orbits such that $r k$ is a multiple of 4 . If $k \equiv 0(\bmod 4)$ this includes all orbits. If $k \equiv 2(\bmod 4)$ the orbit $\left\{\left(\frac{k}{2}, \frac{k}{2}\right)\right\}$ must be excluded. So the trivial factor for $k$ even is

$$
\begin{array}{ll}
\left(1-g(\psi, \rho)^{k} T\right)^{\left\lfloor\frac{k}{2 p}\right\rfloor} & \text { for } k \equiv 2(\bmod 4) \\
\left(1-g(\psi, \rho)^{k} T\right)^{\left\lfloor\frac{k}{2 p}\right\rfloor+1} & \text { for } k \equiv 0(\bmod 4)
\end{array}
$$

In particular, for $p>\frac{k}{2}$ the trivial factor of $M_{k}\left(t^{3}, T\right)$ is 1 if $k \equiv 2(\bmod 4)$ and $\left(1-g(\psi, \rho)^{k} T\right)$ if $k \equiv 0(\bmod 4)$.

We will now consider the case where $g(t)=\sum b_{i} t^{i}$ has $b_{i} \neq 0$ for $i=1, \ldots, d-2$. This includes the generic case where all coefficients of $g(t)$ are non-zero as a special case. Suppose first that $b_{d-1}=0$ (or, equivalently, that $\left.c_{d-1}=0\right) . S_{d-1}(k, J)$ is the set of all $\left(a_{0}, \ldots, a_{d-2}\right) \in \mathbb{Z}_{\geq 0}^{d-1}$ such that $\sum a_{i}=k$ and $\sum a_{i} \zeta^{j i}=0$ for all $j=1, \ldots, d-2$. The system of equations $\left\{\sum_{i} \zeta^{i j} x_{i}=0 \mid j=1, \ldots, d-2\right\}$ has rank $d-2($ since the $(d-2) \times(d-2)$ minors are Vandermonde determinants) and has $(1,1, \ldots, 1)$ as a solution, so all solutions must be of the form $(a, a, \ldots, a)$ modulo $p$ for some $a$. Therefore

$$
\begin{aligned}
F_{d-1}(J ; T): & =\sum_{k=0}^{\infty} \# S_{d-1}(k, J) T^{k} \\
& =\sum_{a=0}^{p-1} \sum_{s_{0}, \ldots, s_{d-2}=0}^{\infty} T^{\left(a+s_{0} p\right)+\cdots+\left(a+s_{d-2} p\right)} \\
& =\sum_{a=0}^{p-1} T^{(d-1) a} \sum_{s_{0}, \ldots, s_{d-2}=0}^{\infty} T^{p\left(s_{0}+\cdots+s_{d-2}\right)} \\
& =\frac{1-T^{(d-1) p}}{\left(1-T^{p}\right)^{d-1}\left(1-T^{d-1}\right)}
\end{aligned}
$$

Suppose now that $b_{d-1} \neq 0$ (or, equivalently, that $c_{d-1} \neq 0$ ). Making the change of variable $\hat{f}(t)=f\left(t-\frac{c_{d-1}}{d c_{d}}\right.$ ) we eliminate the degree $d-1$ term. Moreover, $\mathrm{Ai}_{\hat{f}}=\mathrm{R}^{1} \pi_{t !} \mathcal{L}_{\psi\left(f\left(x-\frac{c_{d-1}}{d c_{d}}\right)+t x\right)}=\mathrm{R}^{1} \pi_{t !} \mathcal{L}_{\psi\left(f(x)+t\left(x+\frac{c_{d-1}}{d c_{d}}\right)\right)}=\mathrm{Ai}_{f} \otimes$ $\mathcal{L}_{\psi\left(\frac{c_{d-1}}{d c_{d}} t\right)}$ and thus $\mathrm{Sym}^{k} \mathrm{Ai}_{f}=\left(\mathrm{Sym}^{k} \mathrm{~A} i_{\hat{f}}\right) \otimes \mathcal{L}_{\psi\left(-\frac{c_{d-1}}{d c_{d}} t\right)}^{\otimes k}$. As a representation of $D_{\infty}$, we have then $\mathrm{Ai}_{f}=[d-$ $1]_{\star}\left(\mathcal{L}_{\psi(\hat{g}(t))} \otimes \mathcal{L}_{\rho^{d}\left(s_{0} t\right)}\right) \otimes \mathcal{L}_{\rho\left(d(d-1) c_{d} / 2\right)} \otimes \mathcal{Q} \otimes \mathcal{L}_{\psi\left(-\frac{c_{d-1}}{d c_{d}} t\right)}=[d-1]_{\star}\left(\mathcal{L}_{\psi\left(\hat{g}(t)-\frac{c_{d-1}}{d c_{d}} t^{d-1}\right)} \otimes \mathcal{L}_{\rho^{d}\left(s_{0} t\right)}\right) \otimes \mathcal{L}_{\rho\left(d(d-1) c_{d} / 2\right)} \otimes \mathcal{Q}$. In other words, $g(t)=\hat{g}(t)-\frac{c_{d-1}}{d c_{d}} t^{d-1}$.

If $p$ divides $k$, the condition $\sum_{i} a_{i} \zeta^{i j}$ for $j=d-1$ is void, so both the dimension of $M_{k}(f ; T)$ and the trivial factor at infinity behave as in the $b_{d-1}=0$ case. If $p$ does not divide $k$, the condition $\sum_{i} a_{i} \zeta^{i j}$ does never hold for $j=d-1$, so $S_{d-1}\left(k, J_{\geq j}\right)=\emptyset$ for $j=1, \ldots, d-1$. In particular, the trivial factor of $M_{k}(f ; T)$ is 1 . Furthermore, applying the formula for the degree, we get

$$
\operatorname{deg} M_{k}(f, T)=\frac{1}{d-1}\left(\left(\begin{array}{c}
k+d-2 \\
d-2
\end{array}\right)-\# S_{d-1}(k,\{1\})\right) .
$$

As a final example, suppose that $d-1$ is prime and $p$ is a multiplicative generator of $\mathbb{F}_{d-1}$. In this case, all non-trivial $(d-1)$-th roots of unity are conjugate over $\mathbb{F}_{p}$, so $a_{0}+a_{1} \zeta+\cdots+a_{d-2} \zeta^{d-2}=0$ if and only if $a_{0}+a_{1} \zeta^{j}+\cdots+a_{d-2} \zeta^{(d-2) j}=0$ for any $j=1,2, \ldots, d-2$. Therefore $S_{d-1}(k,\{1\})=S_{d-1}(k, J)$ for every $J \subset \mathbb{Z}$ such that $J \cap(d-1) \mathbb{Z}=\emptyset$. As in the previous example, we conclude that, if $c_{d-1}=0$,

$$
F_{d-1}\left(J_{\geq j} ; T\right)=\frac{1-T^{(d-1) p}}{\left(1-T^{p}\right)^{d-1}\left(1-T^{d-1}\right)}
$$

for every $j \in J$. By corollary 3.4 , the degree of $M_{k}(f ; T)$ is the $k$-th coefficient of the power series expansion of $\frac{1}{(d-1)(1-T)^{d-1}}-\frac{1}{d-1} \cdot \frac{1-T^{(d-1) p}}{\left(1-T^{p}\right)^{d-1}\left(1-T^{d-1}\right)} \sum_{j \in J} h(j)=\frac{1}{(d-1)(1-T)^{d-1}}-\frac{d}{d-1} \cdot \frac{1-T^{(d-1) p}}{\left(1-T^{p}\right)^{d-1}\left(1-T^{d-1}\right)}$.

If $c_{d-1} \neq 0$ we have, as in the previous example, the same formula for the degree if $k$ is a multiple of $p$, and the $k$-th coefficient in the power series expansion of

if $k$ is prime to $p$.

$$
\frac{1}{(d-1)(1-T)^{d-1}}-\frac{1}{d-1} \cdot \frac{1-T^{(d-1) p}}{\left(1-T^{p}\right)^{d-1}\left(1-T^{d-1}\right)}
$$




\section{The $p$-adic point-of-view}

In the previous section, we considered the rational function

$$
M_{k}(f, T)=\frac{\operatorname{det}\left(1-\operatorname{Frob} T \mid \mathrm{H}_{c}^{1}\left(\mathbb{A}_{\mathbb{F}_{q}}^{1}, \operatorname{Sym}^{k} \mathrm{Ai}_{f}\right)\right)}{\operatorname{det}\left(1-\operatorname{Frob} T \mid \mathrm{H}_{c}^{2}\left(\mathbb{A}_{\mathbb{F}_{q}}^{1}, \operatorname{Sym}^{k} \mathrm{Ai}_{f}\right)\right)}
$$

from the $\ell$-adic point-of-view. In this section, we use techniques in Dwork theory to describe and analyze $M_{k}(f, T)$ $p$-adically. The motivation for this approach is to provide estimates for the $p$-adic absolute values of the zeros and poles of $M_{k}(f, T)$.

\section{1 $p$-adic interpretation of $M_{k}(f, T)$}

Let $\mathbb{F}_{q}$ be the finite field with $q=p^{a}$ elements. Abusing notation, we will write $f(t, x)$ for the polynomial $f(x)+t x$.

Dwork's theory provides a way to represent the function $M_{k}(f, T) p$-adic homologically. This is accomplished by defining "Frobenius" operators, both denoted by $\bar{\beta}$, which act on certain homology spaces $H_{1, k}$ and $H_{0, k}$, so that

$$
M_{k}(f, T)=\frac{\operatorname{det}\left(1-\bar{\beta} T \mid H_{1, k}\right)}{\operatorname{det}\left(1-q \bar{\beta} T \mid H_{0, k}\right)} .
$$

In this form, estimates for the $p$-adic absolute values of the zeros and poles of $M_{k}(f, T)$ may be derived from an analysis of $\bar{\beta}$ on the homology spaces $H_{0, k}$ and $H_{1, k}$. This analysis is carried out in section 6.2 For now, let us demonstrate how Dwork's theory may be used to derive the above homological description of $M_{k}(f, T)$.

$p$-adic Spaces. We begin by fixing some notation. Let $\mathbb{C}_{p}$ be the completion of an algebraic closure of $\mathbb{Q}_{p}$. The valuation on $\mathbb{Q}_{p}$ is normalized such that $\operatorname{ord}_{p}(p)=1$. Let $\mathbb{Q}_{q}$ denote the unramified extension of $\mathbb{Q}_{p}$ of degree $a$. Let $\pi \in \mathbb{C}_{p}$ be a root of the Artin-Hasse series $\sum_{i=0}^{\infty} \frac{t^{p^{i}}}{p^{i}}$ with $\operatorname{ord}_{p}(\pi)=\frac{1}{p-1}$. Notice that $\mathbb{Q}_{p}(\pi)$ is a totally ramified extension of $\mathbb{Q}_{p}$ of degree $p-1$. Let $\mathbb{Q}_{q}(\pi)$ denote the compositum in $\mathbb{C}_{p}$ of the fields $\mathbb{Q}_{q}$ and $\mathbb{Q}_{p}(\pi)$, and denote its ring of integers by $\mathbb{Z}_{q}[\pi]$. Note that the residue class field of $\mathbb{Z}_{q}[\pi]$ is $\mathbb{F}_{q}$. Let $\tau \in \operatorname{Gal}\left(\mathbb{Q}_{q}(\pi) / \mathbb{Q}_{p}(\pi)\right)$ be a lifting of the Frobenius map $x \mapsto x^{p}$ in $\operatorname{Gal}\left(\mathbb{F}_{q} / \mathbb{F}_{p}\right)$ such that $\tau(\pi)=\pi$.

Dwork's "splitting function" provides a bridge between the finite field $\mathbb{F}_{q}$ and the $p$-adic field $\mathbb{C}_{p}$, by describing exponential sums over finite fields $p$-adic analytically. Let $E(t):=\exp \left(\sum_{i=0}^{\infty} \frac{t^{p^{i}}}{p^{i}}\right)$. Define $\pi_{l}:=\sum_{i=0}^{l} \frac{\pi^{p^{i}}}{p^{i}}$ and note that $\operatorname{ord}_{p}\left(\pi_{l}\right) \geq \frac{p^{l+1}}{p-1}-l-1$. Dwork's infinite splitting function is defined as $\theta(t):=E(\pi t)=\sum_{i=0}^{\infty} \lambda_{i} t^{i}$. Observe that each coefficient satisfies $\operatorname{ord}_{p}\left(\lambda_{i}\right) \geq \frac{i}{p-1}$.

Next, we need to describe the function spaces that our "Frobenius" operators will act upon. In order to obtain the best possible estimates for the zeros and poles of the $L$-function, the functions in these spaces will have specific growth conditions that fit our particular polynomial $f(t, x)$. These are described as follows. Let $b$ and $b^{\prime}$ be two positive real numbers. Define functions $w_{0}, w_{1}: \mathbb{Z}_{\geq 0} \rightarrow \frac{1}{d} \mathbb{Z}$ by

$$
w_{0}(n):=\frac{n}{d} \quad \text { and } \quad w_{1}(n):=\left(\frac{d-1}{d}\right) n .
$$

With $\rho \in \mathbb{R}$, define the $p$-adic spaces

$$
\begin{aligned}
L(b ; \rho) & :=\left\{\sum_{n=0}^{\infty} B_{n} t^{n} \mid B_{n} \in \mathbb{Z}_{q}[\pi], \operatorname{ord}_{p}\left(B_{n}\right) \geq b w_{1}(n)+\rho \text { for all } n \geq 0\right\} \\
L(b) & :=\bigcup_{\rho \in \mathbb{R}} L(b ; \rho) \\
\mathcal{K}\left(b^{\prime}, b ; \rho\right) & :=\left\{\sum_{m=0}^{\infty} B_{m} x^{m} \mid B_{m} \in L\left(b^{\prime} ; b w_{0}(m)+\rho\right) \text { for all } m \geq 0\right\} \\
\mathcal{K}\left(b^{\prime}, b\right) & :=\bigcup_{\rho \in \mathbb{R}} \mathcal{K}\left(b^{\prime}, b ; \rho\right) \\
\mathcal{K}\left(b^{\prime}, b\right)^{\bullet} & :=x \mathcal{K}\left(b^{\prime}, b\right) .
\end{aligned}
$$

Notice that the space $\mathcal{K}\left(b^{\prime}, b\right)$ is indeed suited for our polynomial as follows. Let $\hat{f}(t, x) \in \mathbb{Z}_{q}[t, x]$ denote the Teichmüller lifting of $f(t, x) \in \mathbb{F}_{q}[t, x]$. Then $\hat{f} \in \mathcal{K}\left(\frac{p}{p-1}, \frac{p}{p-1} ;-\frac{p}{p-1}\right)$. 
Relative homology. In order to define relative homology we need to define a twisted differential operator on $\mathcal{K}\left(b^{\prime}, b\right)$. The twisting is necessary since it allows the differential operator to commute with the Frobenius operator $\alpha$, as seen below. Writing $\hat{f}(t, x)=x^{d}+\sum_{n=0}^{d-1} \hat{a}_{n} x^{n}+t x$, define

$$
\begin{aligned}
F(t, x) & :=\theta\left(x^{d}\right) \theta(t x) \prod_{n=0}^{d-1} \theta\left(\hat{a}_{n} x^{n}\right) \in \mathbb{Z}_{q}[\pi][[t, x]] \\
F_{a}(t, x) & :=\prod_{i=0}^{a-1} F^{\tau^{i}}\left(t^{p^{i}}, x^{p^{i}}\right) \in \mathbb{Z}_{q}[\pi][[t, x]] .
\end{aligned}
$$

Observe that

$$
F(t, x) \in \mathcal{K}\left(b^{\prime} / p, b / p ; 0\right) \quad \text { and } \quad F_{a}(t, x) \in \mathcal{K}\left(b^{\prime} / q, b / q ; 0\right)
$$

for all real numbers $b^{\prime} \leq b \leq p /(p-1)$. Next, we define a function $G(t, x)$ such that

$$
F(t, x)=\frac{G(t, x)}{G^{\tau}\left(t^{p}, x^{p}\right)} .
$$

Using this equation recursively, we see that $G(t, x)$ must be defined by

$$
G(t, x):=\prod_{j=0}^{\infty} F^{\tau^{j}}\left(t^{p^{j}}, x^{p^{j}}\right) \in \mathbb{Z}_{p}[\pi][[t, x]] .
$$

Set $\hat{f}_{x}:=x \frac{\partial}{\partial x} \hat{f}(t, x)$. Define a (twisted) differential operator on $\mathcal{K}\left(b^{\prime}, b\right)$ by

$$
\begin{aligned}
D(t): & =\frac{1}{G(t, x)} \circ x \frac{\partial}{\partial x} \circ G(t, x) \\
& =x \frac{\partial}{\partial x}+H(t, x)
\end{aligned}
$$

where

$$
H(t, x):=\sum_{j=0}^{\infty} \pi_{j} p^{j} \hat{f}_{x}^{\tau^{j}}\left(t^{p^{j}}, x^{p^{j}}\right) .
$$

Note, $H$ acts by multiplication and $H \in \mathcal{K}\left(\frac{p}{p-1}, \frac{p}{p-1} ;-1\right)$, thus $D(t)$ is an endomorphism of $\mathcal{K}\left(b^{\prime}, b\right)$. Using this operator, we may define the relative homology spaces

$$
\mathcal{H}_{0}:=\operatorname{ker}\left(D(t) \mid \mathcal{K}\left(b^{\prime}, b\right)\right) \quad \text { and } \quad \mathcal{H}_{1}:=\mathcal{K}\left(b^{\prime}, b\right)^{\bullet} / D(t) \mathcal{K}\left(b^{\prime}, b\right) .
$$

In the introduction of this paper, the space $\mathcal{H}_{1}$ was denoted by $\mathrm{Ai}_{f}$. Now, the above notation can be a bit ambiguous at times. That is, there are times when we will need to keep track of both $t$ and the constants $b^{\prime}$ and $b$ in the homology spaces $\mathcal{H}_{1}$ and $\mathcal{H}_{0}$. Thus, we will often denote $\mathcal{H}_{1}$ by $\mathcal{H}_{1, t}\left(b^{\prime}, b\right)$. In general, $\mathcal{H}_{1, t^{n}}\left(b_{1}, b_{2}\right)$ means $\mathcal{K}\left(b_{1}, b_{2}\right)^{\bullet} / D\left(t^{n}\right) \mathcal{K}\left(b_{1}, b_{2}\right)$ where $D\left(t^{n}\right):=x \frac{\partial}{\partial x}+H\left(t^{n}, x\right)$.

Symmetric powers of relative homology. (cf. Robba [23]) The eigenvalues of the Frobenius operators which act on the relative homology spaces $\mathcal{H}_{1}$ and $\mathcal{H}_{0}$ consist of power series in the variable $t$ that, when specialized to some Teichmüller representative of $\bar{t} \in \overline{\mathbb{F}}_{p}$, produce the reciprocal roots $\pi_{1}(\bar{t}), \ldots, \pi_{d-1}(\bar{t})$ of the $L$-function attached to $f$ over $\mathbb{A}^{1}$, as mentioned in the introduction. Now, in the definition of $M_{k}(f, T)$, a type of symmetric product of these roots is presented. A homological description of this symmetric product is obtained by taking the symmetric power of relative homology. This we will now do. Let $\mathcal{H}_{1}^{(k)}:=S y m^{k} \mathcal{H}_{1}$ denote the $k$-th symmetric power of $\mathcal{H}_{1}$ over $L\left(b^{\prime}\right)$. As we shall see in Theorem 6.4 below, $\mathcal{H}_{1}$ is a free $L\left(b^{\prime}\right)$-module with basis $\left\{x^{i}\right\}_{i=1}^{d-1}$ while $\mathcal{H}_{0}=0$. Thus, $\mathcal{H}_{1}^{(k)}$ is a free $L\left(b^{\prime}\right)$-module with basis $\left\{e_{1}^{i_{1}} \cdots e_{d-1}^{i_{d-1}}\right\}_{i_{1}+\cdots+i_{d-1}=k}$ where $e_{i}:=x^{i}$. For notational convenience, we will denote $e_{1}^{i_{1}} \cdots e_{d-1}^{i_{d-1}}$ by $e^{\mathbf{i}}$ with $\mathbf{i}:=\left(i_{1}, \ldots, i_{d-1}\right)$. For $\rho \in \mathbb{R}$, define

$$
\mathcal{H}_{1}^{(k)}\left(b^{\prime}, b ; \rho\right):=\left\{\sum_{\substack{\mathbf{i}:=\left(i_{1}, \ldots, i_{d-1}\right) \in \mathbb{Z}_{\geq 0}^{d-1} \\ i_{1}+\cdots+i_{d-1}=k}} B_{\mathbf{i}} e^{\mathbf{i}} \mid B_{\mathbf{i}} \in L\left(b^{\prime} ; b w_{0}(\mathbf{i})+\rho\right)\right\}
$$

where $w_{0}(\mathbf{i})$ is defined by

$$
w_{0}(\mathbf{i}):=w_{0}\left(i_{1}, \ldots, i_{d-1}\right):=\sum_{j=1}^{d-1} i_{j} w_{0}(j) .
$$


Note that

$$
\mathcal{H}_{1}^{(k)}=\mathcal{H}_{1}^{(k)}\left(b^{\prime}, b\right):=\bigcup_{\rho \in \mathbb{R}} \mathcal{H}_{1}^{(k)}\left(b^{\prime}, b ; \rho\right)
$$

Next, we define a differential operator on $\mathcal{H}_{1}^{(k)}$ as follows. With $\hat{f}_{t}(t, x):=t \frac{\partial}{\partial t} \hat{f}(t, x)$, define

$$
\begin{aligned}
\partial: & =\frac{1}{G(t, x)} \circ t \frac{\partial}{\partial t} \circ G(t, x) \\
& =t \frac{\partial}{\partial t}+W(t, x)
\end{aligned}
$$

where

$$
W(t, x):=\sum_{j=0}^{\infty} \pi_{j} p^{j} \hat{f}_{t}^{\tau^{j}}\left(t^{p^{j}}, x^{p^{j}}\right)
$$

Note, $W(t, x) \in \mathcal{K}\left(\frac{p}{p-1}, \frac{p}{p-1} ;-1\right)$ and so $\partial$ is an endomorphism of $\mathcal{K}\left(b^{\prime}, b\right)$. Now, since $\partial$ commutes with $D(t)$ as endomorphisms of $\mathcal{K}\left(b^{\prime}, b\right)$, and its image lies in $x \mathcal{K}\left(b^{\prime}, b\right)$, it induces an operator on relative homology $\partial: \mathcal{H}_{1} \rightarrow \mathcal{H}_{1}$. We may extend $\partial$ to $\mathcal{H}_{1}^{(k)}$ by extending linearly the following action: for the product $u_{1} \cdots u_{k} \in \mathcal{H}_{1}^{(k)}$ define

$$
\partial\left(u_{1} \cdots u_{k}\right):=\sum_{j=1}^{k} u_{1} \cdots \hat{u}_{j} \cdots u_{k} \partial\left(u_{j}\right)
$$

We will sometimes denote $\partial$ by $\partial_{t}$ to indicate that it is an endomorphism of $\mathcal{H}_{1, t}^{(k)}$. Define the homology spaces

$$
H_{0, k}:=\operatorname{ker}\left(\partial \mid \mathcal{H}_{1}^{(k)}\right) \quad \text { and } \quad H_{1, k}:=t \mathcal{H}_{1}^{(k)} / \partial \mathcal{H}_{1}^{(k)} \text {. }
$$

In terms of $\ell$-adic theory, these are the $p$-adic versions of $\mathrm{H}_{c}^{2}\left(\mathbb{A}_{\overline{\mathbb{F}}_{q}}^{1}, \operatorname{Sym}^{k} \mathrm{Ai}_{f}\right)$ and $\mathrm{H}_{c}^{1}\left(\mathbb{A}_{\overline{\mathbb{F}}_{q}}^{1}, \operatorname{Sym}^{k} \mathrm{Ai}_{f}\right)$, respectively.

Frobenius map. Now that we have created the appropriate spaces, let us define the Frobenius operators. There will be two Frobenius operators involved, $\bar{\alpha}(t)$ which is defined on relative homology whose eigenvalues are power series in $t$ and equal $\pi_{j}(\bar{t})$ when $t$ is specialized, and another $\bar{\beta}$ which will give the homological description of $M_{k}(f, T)$. We begin with the definition of $\bar{\alpha}$.

Define the Cartier operator

$$
\psi_{x}: \mathcal{K}\left(b^{\prime}, b / p\right) \rightarrow \mathcal{K}\left(b^{\prime}, b\right) \quad \text { by } \quad \sum_{m=0}^{\infty} B_{m} x^{m} \longmapsto \sum_{m=0}^{\infty} B_{p m} x^{m} .
$$

Next, define the Dwork operator, which so far we have been calling the "Frobenius operator", by (recall, $q=p^{a}$ )

$$
\alpha(t):=\psi_{x}^{a} \circ F_{a}(t, x): \mathcal{K}\left(b^{\prime}, b\right) \rightarrow \mathcal{K}\left(b^{\prime} / q, b\right) .
$$

Since $\alpha(t) \circ D(t)=q D\left(t^{q}\right) \circ \alpha(t), \alpha(t)$ induces a map on relative homology

$$
\bar{\alpha}(t): \mathcal{H}_{1, t}\left(b^{\prime}, b\right) \rightarrow \mathcal{H}_{1, t^{q}}\left(b^{\prime} / q, b\right) .
$$

We may extend this map to the symmetric powers of relative homology, which we will denote by $\bar{\alpha}^{(k)}(t)$ : $\mathcal{H}_{1, t}^{(k)}\left(b^{\prime}, b\right) \rightarrow \mathcal{H}_{1, t^{q}}^{(k)}\left(b^{\prime} / q, b\right)$. Next, define the Cartier operator $\psi_{t}: \mathcal{H}_{1, t^{p}}^{(k)}\left(b^{\prime} / p, b\right) \rightarrow \mathcal{H}_{1, t}^{(k)}\left(b^{\prime}, b\right)$ by

$$
\left(\sum_{n=0}^{\infty} B_{\mathbf{i}, n} t^{n}\right) e^{\mathbf{i}} \mapsto\left(\sum_{n=0}^{\infty} B_{\mathbf{i}, p n} t^{n}\right) e^{\mathbf{i}}
$$

where we have used the fixed basis in 6 to describe the elements of $\mathcal{H}_{1}^{(k)}$. Finally, define the Dwork operator

$$
\beta:=\psi_{t}^{a} \circ \bar{\alpha}^{(k)}(t): \mathcal{H}_{1, t}^{(k)}\left(b^{\prime}, b\right) \rightarrow \mathcal{H}_{1, t}^{(k)}\left(b^{\prime}, b\right) .
$$

Since $\beta \circ \partial=q \partial \circ \beta, \beta$ induces linear maps

$$
\bar{\beta}: H_{0, k} \rightarrow H_{0, k} \quad \text { and } \quad \bar{\beta}: H_{1, k} \rightarrow H_{1, k} .
$$

Using Dwork's trace formula twice, precisely as in [23, we have

$$
M_{k}(f, T)=\frac{\operatorname{det}\left(1-\bar{\beta} T \mid H_{1, k}\right)}{\operatorname{det}\left(1-q \bar{\beta} T \mid H_{0, k}\right)} .
$$




\subsection{Estimates for $p$-adic absolute values of $M_{k}(f, T)$}

We now have a $p$-adic homological representation of the $L$-function $M_{k}(f, T)$ as a quotient of characteristic polynomials of the Frobenius $\bar{\beta}$ acting on $H_{1, k}$ and $H_{0, k}$. In this section, we will focus our attention on understanding the roots of these polynomials, and since $H_{0, k}$ is often trivial, we will focus on $\bar{\beta}$ acting on $H_{1, k}$. The approach we will take in finding estimates for the $p$-adic absolute values of the roots lies in the following key idea. On the chain level, $\beta$ acts on $\mathcal{H}_{1}^{(k)}$, a space consisting of functions with specific growth conditions. Since these growth conditions are estimates on the $p$-adic absolute values of the coefficients of the power series of these functions, if one knows how functions in $\mathcal{H}_{1}^{(k)}$ reduce to elements in homology $H_{1, k}$, perhaps the growth rates of these functions transcends to $p$-adic estimates of the reduced elements in homology. This is Dwork's decomposition theory, and it is demonstrated in equation (7) below. As we will see in this section, once this is assumed we may apply the theory to obtain estimates for the $p$-adic absolute values of $M_{k}(f, T)$.

Define $W: \mathbb{Z}_{\geq 0} \times \mathbb{Z}_{\geq 0}^{d-1} \rightarrow \frac{1}{d} \mathbb{Z}_{\geq 0}$ by

$$
W(n, \mathbf{i}):=w_{1}(n)+w_{0}(\mathbf{i})
$$

where $w_{1}$ and $w_{0}$ were defined in the previous section. For notational convenience, define $\mathcal{M}:=\mathcal{H}_{1, t}^{(k)}$ and $\mathcal{M}\left(b^{\prime}, b ; \rho\right):=\mathcal{H}_{1, t}^{(k)}\left(b^{\prime}, b ; \rho\right)$, and $\mathcal{N}:=t \mathcal{H}_{1, t}^{(k)}$ and $\mathcal{N}\left(b^{\prime}, b ; \rho\right):=\left(t \mathcal{H}_{1, t}^{(k)}\right) \cap \mathcal{H}_{1, t}^{(k)}\left(b^{\prime}, b ; \rho\right)$. In general, if $V$ is a subset of $\mathcal{M}$ define $V\left(b^{\prime}, b ; \rho\right):=V \cap \mathcal{M}\left(b^{\prime}, b ; \rho\right)$.

Theorem 6.1 below, the main theorem of this section, provides estimates for the $p$-adic absolute values of the roots of $M_{k}(f, T)$. Define the $q$-adic valuation $\operatorname{ord}_{q}(\cdot):=\frac{1}{a} \operatorname{ord}_{p}(\cdot)$.

Theorem 6.1. Let $p$ be a prime number such that $(p, d)=1$. Suppose there exists a free, finite rank $\mathbb{Z}_{q}[\pi]$ submodule $V$ of $\mathcal{N}$ with basis $\Gamma:=\left\{t^{n} e^{\mathbf{i}}\right\}_{(n, \mathbf{i}) \in A}$ for some index $A$ such that, with $b:=p /(p-1)$,

$$
\mathcal{N}(b, b ; 0) \subset V(b, b ; 0) \oplus \partial \mathcal{M}(b, b ; \epsilon)
$$

for some $\epsilon \in \mathbb{R}_{>0}$. Then, writing $\operatorname{det}_{\mathbb{Q}_{q}(\pi)}\left(1-\bar{\beta} T \mid H_{1, k}\right)=\sum_{m=0}^{\operatorname{dim}_{\mathbb{Q}_{q}(\pi)}\left(H_{1, k}\right)} c_{m} T^{m}$, we have

$$
\operatorname{ord}_{q}\left(c_{m}\right) \geq \min \left\{\sum_{j=1}^{m} W\left(n^{(j)}, \mathbf{i}^{(j)}\right)\right\}
$$

where the minimum runs over all sets consisting of $m$ distinct elements of the form $(n, \mathbf{i}) \in A$. Equivalently, the q-adic Newton polygon of $\operatorname{det}_{\mathbb{Q}_{q}(\pi)}\left(1-\bar{\beta} T \mid H_{1, k}\right)$ lies on or above the lower convex hull of the points

$$
\left(\sum_{N=0}^{R} r_{N}, \frac{1}{d} \sum_{N=0}^{R} N r_{N}\right) \quad R=0,1, \ldots, \operatorname{dim}_{\mathbb{Q}_{q}(\pi)}\left(H_{1, k}\right),
$$

where $r_{N}:=\#\{(n, \mathbf{i}) \in A \mid W(n, \mathbf{i})=N / d\}$.

Before proceeding to the proof of this theorem, let us give an example of it. In [15], the function $M_{k}(f, T)$ was studied for the cubic family $f(t, x)=x^{3}+t x$. In particular, $\mathcal{N}=t \mathcal{H}_{1}^{(k)}$ was essentially shown to satisfy $(7)$ for some $V$. Consequently, using Theorem 6.1, we have the following result, which was conjectured in loc.cit..

Corollary 6.2. Let $p \geq 5$ be a prime number. For the cubic family $f(t, x):=x^{3}+t x$, when the symmetric power $k$ is odd and $k<p$, then $M_{k}\left(x^{3}, T\right)$ is a polynomial. Furthermore, writing $M_{k}\left(x^{3}, T\right)=\operatorname{det}_{\mathbb{Q}_{q}(\pi)}\left(1-\bar{\beta} T \mid H_{1, k}\right)=$ $\sum_{m=0}^{\operatorname{dim}_{\mathbb{Q}_{q}(\pi)}\left(H_{1, k}\right)} c_{m} T^{m}$, we have

$$
\operatorname{ord}_{q}\left(c_{m}\right) \geq \frac{1}{3}\left(m^{2}+m+k m\right) .
$$

Proof of Corollary. By Corollary 6.15 below and [15, Section 6.2], the $\mathbb{Z}_{q}[\pi]$-submodule $V$ defined by the $\mathbb{Z}_{q}[\pi]$ span of the set $\left\{t e_{1}^{k-2 i} e_{2}^{2 i}\right\}_{i=0}^{\frac{k-1}{2}} \subset \mathcal{N}$ satisfies $(7)$ when $k$ is odd and $k<p$. Thus, $A=\{(1,(k-2 i, 2 i))\}_{i=0}^{\frac{k-1}{2}}$, and so by Theorem 6.1.

$$
\begin{aligned}
\operatorname{ord}_{q}\left(c_{m}\right) & \geq \sum_{j=0}^{m-1}\left(w_{1}(1)+w_{0}(k-2 i, 2 i)\right) \\
& =m w_{1}(1)+k m w_{0}(1)+\left(w_{0}(2)-w_{0}(1)\right) m(m-1) .
\end{aligned}
$$

The result follows since $w_{1}(n):=2 n / 3$ and $w_{0}(n):=n / 3$. 
The main result in Section 6.4 below will be to reduce the hypothesis (7) to a similar hypothesis which we believe is attainable. It is expected that this similar hypothesis will hold under rather general conditions on the prime $p$, the degree $d$, and the symmetric power $k$. However, it is also expected that this similar hypothesis will fail just as often, yet (7) will still hold. The conditions under which the weaker hypothesis is valid is currently under investigation.

The rest of this section is devoted to the proof of Theorem 6.1, whose argument closely follows that of Dwork's [9, §7] and Adolphson-Sperber's [2. The proof rests on relating the Newton polygon of $\bar{\beta}$ to another operator, $\bar{\beta}_{1}$, whose Newton polygon is much easier to estimate due to the Dwork decomposition of $\mathcal{N}$ given in 77 . The reason is that Dwork decomposition allows us to work on the chain level, where the operator $\beta_{1}$ acts in an easily understood way. Once estimates on $\beta_{1}$ are found on the chain level Dwork decomposition provides estimates in homology of $\bar{\beta}_{1}$.

For $b^{\prime} \leq b$ and $b \leq p /(p-1)$, define $\alpha_{1}: \mathcal{K}\left(b^{\prime}, b\right) \rightarrow \mathcal{K}\left(b^{\prime} / p, b\right)$ by

$$
\begin{aligned}
\alpha_{1}: & =\tau^{-1} \circ \psi_{x} \circ F(t, x) \\
& =\frac{1}{G\left(t^{p}, x\right)} \circ \tau^{-1} \circ \psi_{x} \circ G(t, x) .
\end{aligned}
$$

Notice that $\alpha_{1} \circ D(t)=p D\left(t^{p}\right) \circ \alpha_{1}$, and so $\alpha_{1}$ induces a map

$$
\bar{\alpha}_{1}(t): \mathcal{H}_{1, t}\left(b^{\prime}, b\right) \rightarrow \mathcal{H}_{1, t^{p}}\left(b^{\prime} / p, b\right) .
$$

On $\mathcal{K}\left(b^{\prime}, b\right)$, since

$$
\begin{aligned}
\alpha(t) & =\psi_{x}^{a} \circ F_{a}(t, x) \\
& =\psi_{x}^{a} \circ F^{\tau^{a-1}}\left(t^{p^{a-1}}, x^{p^{a-1}}\right) \cdots F^{\tau}\left(t^{p}, x^{p}\right) F(t, x) \\
& =\left(\tau^{-1} \circ \psi_{x} \circ F\left(t^{p^{a-1}}, x\right)\right) \circ \cdots \circ\left(\tau^{-1} \circ \psi_{x} \circ F\left(t^{p}, x\right)\right) \circ\left(\tau^{-1} \circ \psi_{x} \circ F(t, x)\right) \\
& =\alpha_{1}\left(t^{p^{a-1}}\right) \circ \cdots \circ \alpha_{1}\left(t^{p}\right) \circ \alpha_{1}(t),
\end{aligned}
$$

it follows that

$$
\bar{\alpha}(t)=\bar{\alpha}_{1}\left(t^{p^{a-1}}\right) \circ \cdots \circ \bar{\alpha}_{1}\left(t^{p}\right) \circ \bar{\alpha}_{1}(t)
$$

We also have the property that

$$
\psi_{t} \circ \bar{\alpha}_{1}\left(t^{p}\right)=\bar{\alpha}_{1}(t) \circ \psi_{t} .
$$

Consequently, with $\beta_{1}:=\psi_{t} \circ \operatorname{Sym}^{k}\left(\bar{\alpha}_{1}(t)\right): \mathcal{H}_{1, t}^{(k)}\left(b^{\prime}, b\right) \rightarrow \mathcal{H}_{1, t}^{(k)}\left(b^{\prime}, b\right)$, we have the relation

$$
\begin{aligned}
\beta_{1}^{a} & =\psi_{t}^{a} \circ \operatorname{Sym}^{k}\left(\bar{\alpha}_{1}\left(t^{p^{a-1}}\right) \circ \cdots \circ \bar{\alpha}_{1}\left(t^{p}\right) \circ \bar{\alpha}_{1}(t)\right) \\
& =\psi_{t}^{a} \circ \operatorname{Sym}^{k}(\bar{\alpha}(t)) \\
& =\beta
\end{aligned}
$$

where we have used $\sqrt{9}$ for the first equality and $(8)$ for the second. Since $\operatorname{det}_{\mathbb{Q}_{q}(\pi)}\left(1-\bar{\beta} T \mid H_{1, k}\right) \in \mathbb{Q}_{p}(\pi)$, we have that

$$
\begin{aligned}
\left(\operatorname{det}_{\mathbb{Q}_{q}(\pi)}\left(1-\bar{\beta} T \mid H_{1, k}\right)\right)^{a} & =\operatorname{Norm}_{\mathbb{Q}_{q}(\pi) / \mathbb{Q}_{p}(\pi)} \operatorname{det}_{\mathbb{Q}_{q}(\pi)}\left(1-\bar{\beta} T \mid H_{1, k}\right) \\
& =\operatorname{det}_{\mathbb{Q}_{p}(\pi)}\left(1-\bar{\beta} T \mid H_{1, k}\right) .
\end{aligned}
$$

Thus,

$$
\begin{aligned}
\left(\operatorname{det}_{\mathbb{Q}_{q}(\pi)}\left(1-\bar{\beta} T^{a} \mid H_{1, k}\right)\right)^{a} & =\operatorname{det}_{\mathbb{Q}_{p}(\pi)}\left(1-\bar{\beta} T^{a} \mid H_{1, k}\right) \\
& =\operatorname{det}_{\mathbb{Q}_{p}(\pi)}\left(1-\bar{\beta}_{1}^{a} T^{a} \mid H_{1, k}\right) \\
& =\prod_{\zeta^{a}=1} \operatorname{det}_{\mathbb{Q}_{p}(\pi)}\left(1-\zeta \bar{\beta}_{1} T \mid H_{1, k}\right) .
\end{aligned}
$$

Counting multiplicities, let $m_{i}$ denote the number of reciprocal roots of $\operatorname{det}_{\mathbb{Q}_{p}(\pi)}\left(1-\bar{\beta}_{1} T \mid H_{1, k}\right)$ which have slope $s_{i}$; note, we say $\lambda$ has slope $s_{i}$ if $\operatorname{ord}_{p}(\lambda)=s_{i}$. Then, from $\sqrt[10]{ },\left(\operatorname{det}_{\mathbb{Q}_{q}(\pi)}\left(1-\bar{\beta} T^{a} \mid H_{1, k}\right)\right)^{a}$ has $a m_{i}$ reciprocal roots of slope $s_{i}$, and so $\operatorname{det}_{\mathbb{Q}_{q}(\pi)}\left(1-\bar{\beta} T^{a} \mid H_{1, k}\right)$ has $m_{i}$ reciprocal roots with slope $s_{i}$. We conclude that $\operatorname{det}_{\mathbb{Q}_{q}(\pi)}\left(1-\bar{\beta} T \mid H_{1, k}\right)$ has $m_{i} / a$ reciprocal roots of slope $a s_{i}$.

Next, for the $q$-adic valuation $\operatorname{ord}_{q}(\cdot):=\frac{1}{a} \operatorname{ord}_{p}(\cdot)$, we will say a root $\lambda$ has $q$-adic slope $s_{i}$ if $\operatorname{ord}_{q}(\lambda)=s_{i}$. Observe that the above paragraph has demonstrated that $\operatorname{det}_{\mathbb{Q}_{q}(\pi)}\left(1-\bar{\beta} T \mid H_{1, k}\right)$ has $m_{i}$ reciprocal roots with 
q-adic slope $s_{i}$ if and only if $\operatorname{det}_{\mathbb{Q}_{p}(\pi)}\left(1-\bar{\beta}_{1} T \mid H_{1, k}\right)$ has $a m_{i}$ reciprocal roots with $p$-adic slope $s_{i}$. In terms of Newton polygons, this means the vertices of the $q$-adic Newton polygon of $\operatorname{det}_{\mathbb{Q}_{q}(\pi)}\left(1-\bar{\beta} T \mid H_{1, k}\right)$ are

$$
(0,0) \text { and }\left(\sum_{i=1}^{n} m_{i}, \sum_{i=1}^{n} m_{i} s_{i}\right) \quad n=1,2, \ldots, \operatorname{dim}_{\mathbb{Q}_{q}(\pi)}\left(H_{1, k}\right)
$$

if and only if the vertices of the $p$-adic Newton polygon of $\operatorname{det}_{\mathbb{Q}_{p}(\pi)}\left(1-\bar{\beta}_{1} T \mid H_{1, k}\right)$ are

$$
(0,0) \quad \text { and }\left(\sum_{i=1}^{n} a m_{i}, \sum_{i=1}^{n} a m_{i} s_{i}\right) \quad n=1,2, \ldots, \operatorname{dim}_{\mathbb{Q}_{p}(\pi)}\left(H_{1, k}\right) .
$$

Using this relation, any lower bound for the $p$-adic Newton polygon of the latter may be transformed to a lower bound of the $q$-adic Newton polygon of the former by dividing the coordinates of the vertices by $a$. Let us now concentrate on a lower bound for the $p$-adic Newton polygon of $\operatorname{det}_{\mathbb{Q}_{p}(\pi)}\left(1-\bar{\beta}_{1} T \mid H_{1, k}\right)$.

Define $\mathcal{K}\left(b^{\prime}, b\right)^{\bullet}:=x \mathcal{K}\left(b^{\prime}, b\right)$, and let $\mathcal{V}$ be the $L\left(b^{\prime}\right)$-span of the set $\left\{x, x^{2}, \ldots, x^{d-1}\right\}$ in $\mathcal{K}\left(b^{\prime}, b\right)^{\bullet}$. Define $\mathcal{K}\left(b^{\prime}, b ; \rho\right)^{\bullet}:=\mathcal{K}\left(b^{\prime}, b\right)^{\bullet} \cap \mathcal{K}\left(b^{\prime}, b ; \rho\right)$ and $\mathcal{V}\left(b^{\prime}, b ; \rho\right):=\mathcal{V} \cap \mathcal{K}\left(b^{\prime}, b ; \rho\right)$. By our hypothesis on the prime $p$, we will prove in the following section the Dwork decomposition

$$
\mathcal{K}\left(b^{\prime}, b ; 0\right)^{\bullet} \subset \mathcal{V}\left(b^{\prime}, b ; 0\right) \oplus D(t) \mathcal{K}\left(b^{\prime}, b ; e\right),
$$

where $e:=b-\frac{1}{p-1}$. Consequently, $\mathcal{K}\left(b^{\prime}, b\right)^{\bullet}=\mathcal{V} \oplus D(t) \mathcal{K}\left(b^{\prime}, b\right)$, so we may identify $\mathcal{H}_{1}$ with $\mathcal{V}$, a free $L\left(b^{\prime}\right)$-module of rank $d-1$ with basis $\left\{x, x^{2}, \ldots, x^{d-1}\right\}$. Consequently, $\mathcal{H}_{1}^{(k)}$ is a free $L\left(b^{\prime}\right)$-module with basis $\left\{e_{1}^{i_{1}} \cdots e_{d-1}^{i_{d-1}}:=e^{\mathrm{i}}\right\}$, where $\mathbf{i}=\left(i_{1}, \ldots, i_{d-1}\right)$, each $i_{j}$ is a nonnegative integer satisfying $i_{1}+\cdots+i_{d-1}=k$, and $e_{j}:=x^{j}$.

Set $b=\frac{p}{p-1}$. For $i=1, \ldots, d-1, x^{i} \in \mathcal{K}\left(\frac{b}{p}, \frac{b}{p} ;-\frac{b}{p} w_{0}(i)\right)^{\bullet}$ and so $F(t, x) x^{i} \in \mathcal{K}\left(\frac{b}{p}, \frac{b}{p} ;-\frac{b}{p} w_{0}(i)\right)^{\bullet}$. Thus, $\alpha_{1}\left(x^{i}\right) \in \mathcal{K}\left(\frac{b}{p}, b ;-\frac{b}{p} w_{0}(i)\right)^{\bullet}$. By 11$]$ we may write this as

$$
\alpha_{1}\left(x^{i}\right)=A_{i, 1} x+\cdots+A_{i, d-1} x^{d-1} \bmod \left(D\left(t^{p}\right) \mathcal{K}\left(\frac{b}{p}, b\right)\right)
$$

where $A_{i, j} \in L\left(\frac{b}{p} ; \frac{b}{p}\left(p w_{0}(j)-w_{0}(i)\right)\right)$.

Let $S\left(i_{1}, \ldots, i_{d-1}\right)$ denote the set of nonnegative integers $\left(l_{s}^{(r)}\right)_{1 \leq s, r \leq d-1}$ that satisfy the system

$$
\begin{gathered}
l_{1}^{(1)}+\cdots+l_{d-1}^{(1)}=i_{1} \\
\vdots \\
l_{1}^{(d-1)}+\cdots+l_{d-1}^{(d-1)}=i_{d-1} .
\end{gathered}
$$

and $T\left(j_{1}, \ldots, j_{d-1}\right)$ denote the set of nonnegative integers $\left(l_{s}^{(r)}\right)_{1 \leq s, r \leq d-1}$ that satisfy the system

$$
\begin{gathered}
l_{1}^{(1)}+\cdots+l_{1}^{(d-1)}=j_{1} \\
\vdots \\
l_{d-1}^{(1)}+\cdots+l_{d-1}^{(d-1)}=j_{d-1} .
\end{gathered}
$$

The $k$-th symmetric power of $\bar{\alpha}_{1}$ acts on the basis $\left\{e^{\mathbf{i}}\right\}$ as follows:

$$
\begin{aligned}
\operatorname{Sym}^{k}\left(\bar{\alpha}_{1}(t)\right) e_{1}^{i_{1}} \cdots e_{d-1}^{i_{d-1}} \\
=\left(\bar{\alpha}_{1}(t) e_{1}\right)^{i_{1}} \cdots\left(\bar{\alpha}_{1}(t) e_{d-1}\right)^{i_{d-1}} \\
=\left(\sum_{j=1}^{r} A_{1, j} e_{j}\right)^{i_{1}} \cdots\left(\sum_{j=1}^{r} A_{d-1, j} e_{j}\right)^{i_{d-1}} \\
=\sum_{\substack{\left(l_{s}^{(r)}\right) \in S\left(i_{1}, \ldots, i_{d-1}\right) \\
=}} \mathbb{Z}_{\substack{\mathbf{j}:=\left(j_{1}, \ldots, j_{d-1}\right) \in \mathbb{Z}_{\geq 0}^{d-1} \\
j_{1}+\cdots+j_{d-1}=k}} B\left(A_{1,1}^{l_{1}^{(1)}} \cdots A_{1, d-1}^{l_{d-1}^{(1)}}\right) \cdots\left(A_{d-1,1}^{l_{1}^{(d-1)}} \cdots A_{d-1, d-1}^{l_{d-1}^{(d-1)}}\right) e_{1}^{j_{1}} \cdots e_{d-1}^{j_{d-1}^{(1)}+\cdots+l_{1}^{(d-1)}} \cdots e_{d-1}^{l_{d-1}^{(1)}+\cdots+l_{d-1}^{(d-1)}} \\
\end{aligned}
$$


where

$$
B(\mathbf{i} ; \mathbf{j}):=\sum_{\left(l_{s}^{(r)}\right) \in S\left(i_{1}, \ldots, i_{d-1}\right) \cap T\left(j_{1}, \ldots, j_{d-1}\right)}\left(\mathbb{Z}_{>0}\right)\left(A_{1,1}^{l_{1}^{(1)}} \cdots A_{1, d-1}^{l_{d-1}^{(1)}}\right) \cdots\left(A_{d-1,1}^{l_{1}^{(d-1)}} \cdots A_{d-1, d-1}^{l_{d-1}^{(d-1)}}\right)
$$

and " $\mathbb{Z}_{>0}$ " is some determinable nonzero positive integer. It follows that $B(\mathbf{i} ; \mathbf{j}) \in L\left(\frac{b}{p} ; \frac{b}{p}\left(p w_{0}(\mathbf{j})-w_{0}(\mathbf{i})\right)\right)$, and so

$$
\operatorname{Sym}^{k}\left(\bar{\alpha}_{1}(t)\right): \mathcal{H}_{1}^{(k)}\left(\frac{b}{p}, \frac{b}{p} ; 0\right) \rightarrow \mathcal{H}_{1}^{(k)}\left(\frac{b}{p}, b ; 0\right) .
$$

Recall, $\mathcal{M}:=\mathcal{H}_{1, t}^{(k)}$ and $\mathcal{N}:=t \mathcal{H}_{1, t}^{(k)}$. We are supposing that there exists a free $\mathbb{Z}_{q}[\pi]$-submodule $\mathcal{V}$ of $\mathcal{N}$ with basis $\Gamma:=\left\{t^{n} e^{\mathbf{i}} \mid(n ; \mathbf{i}) \in A\right\}$ such that

$$
\mathcal{N}(b, b ; 0) \subset \mathcal{V}(b, b ; 0) \oplus \partial \mathcal{M}(b, b ; \epsilon)
$$

for some $\epsilon \in \mathbb{R}$. Now, $\Gamma$ represents a basis of $H_{1, k}$ over $\mathbb{Q}_{q}(\pi)$, but we need to understand the Fredholm determinant of $\bar{\beta}_{1}$ on $H_{1, k}$ viewed as a vector space over $\mathbb{Q}_{p}(\pi)$. To do this recall that $\mathbb{Q}_{q}(\pi)$ is an unramified extension field of $\mathbb{Q}_{p}(\pi)$. We have denoted by $\mathbb{Z}_{q}[\pi]$ the ring of integers of $\mathbb{Q}_{q}(\pi)$ with uniformizer $\pi$ and residue field $\mathbb{F}_{q}$, and $\mathbb{Z}_{p}[\pi]$ the ring of integers of $\mathbb{Q}_{p}(\pi)$ with uniformizer $\pi$ and residue field $\mathbb{F}_{p}$. Let $\left\{\bar{\eta}_{1}, \ldots, \bar{\eta}_{a}\right\}$ be a basis of $\mathbb{F}_{q}$ over $\mathbb{F}_{p}$, and let $\left\{\eta_{1}, \ldots, \eta_{a}\right\}$ be a lifting of this basis to an integral basis of $\mathbb{Q}_{q}(\pi)$ over $\mathbb{Q}_{p}(\pi)$.

Lemma 6.3 (Dwork). The basis $\left\{\eta_{i}\right\}$ has the property of p-adic directness; that is, for any $g \in \mathbb{Q}_{q}(\pi)$, writing $g=h_{1} \eta_{1}+\cdots+h_{a} \eta_{a}$ with $h_{i} \in \mathbb{Q}_{p}(\pi)$, then

$$
\operatorname{ord}_{p}(g)=\min _{i=1, \ldots, a}\left\{\operatorname{ord}_{p}\left(h_{i}\right)\right\} .
$$

Proof. Without loss of generality, we may assume that $\operatorname{ord}_{p}(g)=0$. Set $-c:=(p-1) \min \left\{\operatorname{ord}_{p}\left(h_{i}\right)\right\} \in \mathbb{Z}$. Suppose $c>0$. For any $\xi \in \mathbb{Z}_{q}[\pi]$, denote by $\bar{\xi}$ its image in the residue field $\mathbb{F}_{q}$. Using this notation, we see that

$$
0=\left(\overline{\pi^{c} g}\right)=\left(\overline{\pi^{c} h_{1}}\right) \bar{\eta}_{1}+\cdots+\left(\overline{\pi^{c} h_{a}}\right) \bar{\eta}_{a} \bmod (\pi)
$$

Since $\left\{\bar{\eta}_{i}\right\}$ is a basis of $\mathbb{F}_{q}$, we must have $\overline{\pi^{c} h_{i}}=0$ in $\mathbb{F}_{q}$ for every $i$. Hence, $\pi^{c} h_{i} \in \pi \mathbb{Z}_{q}[\pi]$, and so $h_{i} \in \pi^{1-c} \mathbb{Z}_{q}[\pi]$ for every $i$. Thus, for each $i$ we have

$$
\operatorname{ord}_{p}\left(h_{i}\right) \geq \frac{1-c}{p-1}=\frac{1}{p-1}+\min _{j=1, \ldots, a}\left\{\operatorname{ord}_{p}\left(h_{j}\right)\right\} .
$$

However, since this is not possible we must have $c$ nonnegative. Thus, $-c \geq 0$ which means $\min \left\{\operatorname{ord}_{p}\left(h_{i}\right)\right\} \geq 0=$ $\operatorname{ord}_{p}(g)$. Since we easily have $\operatorname{ord}_{p}(g) \geq \min \left\{\operatorname{ord}_{p}\left(h_{i}\right)\right\}$, we must have equality, proving the lemma.

Since $t^{n} e^{\mathbf{i}} \in \mathcal{M}\left(\frac{b}{p}, \frac{b}{p} ;-\frac{b}{p} W(n, \mathbf{i})\right)$, we have by 13 that $\operatorname{Sym}^{k}\left(\alpha_{1}(t)\right)\left(t^{n} e^{\mathbf{i}}\right) \in \mathcal{N}\left(\frac{b}{p}, b ;-\frac{b}{p} W(n, \mathbf{i})\right)$ and so $\beta_{1}\left(t^{n} e^{\mathbf{i}}\right) \in \mathcal{N}\left(b, b ;-\frac{b}{p} W(n, \mathbf{i})\right)$. By Dwork decomposition 14 , this means

$$
\beta_{1}\left(t^{n} e^{\mathbf{i}}\right)=\sum_{(m, \mathbf{j}) \in \Gamma} C(n, \mathbf{i} ; m, \mathbf{j}) t^{m} e^{\mathbf{j}} \quad \bmod (\partial \mathcal{M})
$$

with

$$
\operatorname{ord}_{p}(C(n, \mathbf{i} ; m, \mathbf{j})) \geq \frac{b}{p}(p W(m, \mathbf{j})-W(n, \mathbf{i})) .
$$

From the lemma above, if $B \in \mathbb{Z}_{q}[\pi]$ satisfies $\operatorname{ord}_{p}(B) \geq \rho$, then writing $B=B_{1} \eta_{1}+\cdots+B_{a} \eta_{a}$ the coefficients satisfy $\operatorname{ord}_{p}\left(B_{i}\right) \geq \rho$. Thus, we may write

$$
C(n, \mathbf{i} ; m, \mathbf{j})=\sum_{r=1}^{a} C(n, \mathbf{i} ; m, \mathbf{j})_{r} \eta_{r}
$$

with $C(n, \mathbf{i} ; m, \mathbf{j})_{r} \in \mathbb{Z}_{p}[\pi]$ and $\operatorname{ord}_{p}\left(C(n, \mathbf{i} ; m, \mathbf{j})_{r}\right) \geq \frac{b}{p}(p W(m, \mathbf{j})-W(n, \mathbf{i}))$. Now, a basis of $H_{1, k}$ over the field $\mathbb{Q}_{p}(\pi)$ is given by $\Gamma^{\prime}:=\left\{\eta_{j} t^{n} e^{\mathbf{i}} \mid j=1, \ldots, a,(n, \mathbf{i}) \in A\right\}$. Thus, for $\eta_{j} t^{n} e^{\mathbf{i}} \in \Gamma^{\prime}$, we have

$$
\begin{aligned}
\beta_{1}\left(\eta_{j} t^{n} e^{\mathbf{i}}\right) & =\tau^{-1}\left(\eta_{j}\right) \sum_{(m, \mathbf{j}) \in \Gamma} C(n, \mathbf{i} ; m, \mathbf{j}) t^{m} e^{\mathbf{j}} \quad \bmod (\partial \mathcal{M}) \\
& =\tau^{-1}\left(\eta_{j}\right) \sum_{(m, \mathbf{j}) \in \Gamma} \sum_{r=1}^{a} C(n, \mathbf{i} ; m, \mathbf{j})_{r} \eta_{r} e^{\mathbf{j}}
\end{aligned}
$$


Writing

$$
\tau^{-1}\left(\eta_{j}\right) \eta_{r}=\sum_{s=1}^{a} b_{s, j, r} \eta_{s} \quad \text { with } b_{s, j, r} \in \mathbb{Z}_{p}[\pi]
$$

then (15) becomes

$$
\bar{\beta}_{1}\left(\eta_{j} t^{n} e^{\mathbf{i}}\right)=\sum_{(m, \mathbf{j}) \in \Gamma} \sum_{s=1}^{a} D(j, n, \mathbf{i} ; s, m, \mathbf{j}) \eta_{s} t^{m} e^{\mathbf{j}}
$$

where

$$
D(j, n, \mathbf{i} ; s, m, \mathbf{j}):=\sum_{r=1}^{a} C(n, \mathbf{i} ; m, \mathbf{j})_{r} b_{s, j, r} .
$$

It follows that

$$
\operatorname{ord}_{p}(D(j, n, \mathbf{i} ; s, m, \mathbf{j})) \geq \frac{b}{p}(p W(m, \mathbf{j})-W(n, \mathbf{i})) .
$$

Writing $\operatorname{det}_{\mathbb{Q}_{p}(\pi)}\left(1-\bar{\beta}_{1} T \mid H_{1, k}\right)=\sum_{m=0}^{\infty} c_{m} T^{m}$ then

$$
c_{m}=(-1)^{m} \sum \sum_{\sigma \in \mathcal{S}_{m}} \operatorname{sgn}(\sigma) \prod_{l=1}^{m} D\left(j_{l}, n_{l}, \mathbf{i}^{(l)} ; j_{\sigma(l)}, n_{\sigma(l)}, \mathbf{i}^{(\sigma(l))}\right),
$$

where $\mathcal{S}_{m}$ is the permutation group on $\{1, \ldots, m\}$ and the outer summation runs over all sets consisting of $m$ distinct elements of the form $(j, n, \mathbf{i})$ where $\eta_{j} t^{n} e^{\mathbf{i}} \in \Gamma^{\prime}$. It follows that

$$
\operatorname{ord}_{p}\left(c_{m}\right) \geq \min \left\{\sum_{l=1}^{m} W\left(n_{l}, \mathbf{i}^{(l)}\right)\right\}
$$

where the minimum runs over all sets consisting of $m$ distinct elements of the form $(j, n, \mathbf{i})$ where $\eta_{j} t^{n} e^{\mathbf{i}} \in \Gamma^{\prime}$.

Let $r_{N}:=\#\left\{t^{n} e^{\mathbf{i}} \in \Gamma \mid W(n, \mathbf{i})=N / d\right\}$. Then there are $a r_{N}$ number of elements $\eta_{j} t^{n} e^{\mathbf{i}} \in \Gamma^{\prime}$ with weight $W(n, \mathbf{i})=N / d$. Thus, if

$$
m=\sum_{N=0}^{R} a r_{N}
$$

then

$$
\operatorname{ord}_{p}\left(c_{m}\right) \geq \sum_{N=0}^{R} a r_{N}\left(\frac{N}{d}\right) .
$$

In other words, the $p$-adic Newton polygon of $\operatorname{det}_{\mathbb{Q}_{p}(\pi)}\left(1-\bar{\beta}_{1} T \mid H_{1, k}\right)$ lies on or above the lower convex hull of the points

$$
\left(\sum_{N=0}^{R} a r_{N}, \sum_{N=0}^{R} a r_{N} \frac{N}{d}\right) \quad R=0,1, \ldots, \operatorname{dim}_{\mathbb{Q}_{p}(\pi)} H_{1, k}
$$

Thus, the $q$-adic Newton polygon of $\operatorname{det}_{\mathbb{Q}_{q}(\pi)}\left(1-\beta T \mid H_{1, k}\right)$ lies on or above the lower convex hull of the points

$$
\left(\sum_{N=0}^{R} r_{N}, \sum_{N=0}^{R} r_{N} \frac{N}{d}\right) \quad R=0,1, \ldots, \operatorname{dim}_{\mathbb{Q}_{q}(\pi)} H_{1, k} .
$$

This finishes the proof of Theorem 6.1

\subsection{Relative Dwork homology}

Integral to the proof of Theorem 6.1 was the Dwork decomposition of relative homology given on (11). The main result of this section is to provide a proof of this result. This proof will closely follow arguments of Dwork's 9 , $\S 7$ and Adolphson-Sperber's [2]. We begin by recalling that

$$
\begin{aligned}
\mathcal{V}\left(b^{\prime}, b ; \rho\right) & :=\left(L\left(b^{\prime}\right) x \oplus \cdots \oplus L\left(b^{\prime}\right) x^{d-1}\right) \cap \mathcal{K}\left(b^{\prime}, b ; \rho\right) \\
\mathcal{V}\left(b^{\prime}, b\right) & :=\bigcup_{\rho \in \mathbb{R}} \mathcal{V}\left(b^{\prime}, b ; \rho\right) .
\end{aligned}
$$

Theorem 6.4. Suppose $(p, d)=1$. Let $b$ and $b^{\prime}$ be real numbers satisfying $b^{\prime} \leq b$ and $\frac{1}{p-1} \leq b \leq \frac{p}{p-1}$. Set $e:=b-\frac{1}{p-1}$. Then 
1. $\mathcal{K}\left(b^{\prime}, b\right)^{\bullet}=\mathcal{V}\left(b^{\prime}, b\right) \oplus D(t) \mathcal{K}\left(b^{\prime}, b\right)$,

2. $\mathcal{K}\left(b^{\prime}, b ; 0\right) \bullet \mathcal{V}\left(b^{\prime}, b ; 0\right) \oplus D(t) \mathcal{K}\left(b^{\prime}, b ; e\right)$,

3. $D(t)$ is injective if $b>\frac{1}{p-1}$,

4. if $g \in \mathcal{K}\left(b^{\prime}, b\right)^{\bullet}$ is divisible by $t^{n}$ and we write $g=\xi+D(t) \zeta$ with $\xi \in \mathcal{V}\left(b^{\prime}, b\right)$ and $\zeta \in \mathcal{K}\left(b^{\prime}, b\right)$, then $t^{n}$ divides $\xi$ and $\zeta$.

The proof of this theorem will consist of a series of lemmas which will comprise the rest of this section.

Lemma 6.5. Suppose $b^{\prime} \leq b$. Then

$$
\mathcal{K}\left(b^{\prime}, b ; 0\right)^{\bullet} \subset \mathcal{V}\left(b^{\prime}, b ; 0\right)+\pi \hat{f}_{x} \mathcal{K}\left(b^{\prime}, b ; e\right) .
$$

Furthermore, if $\xi \in \mathcal{K}\left(b^{\prime}, b\right)^{\bullet}$ is divisible by $t^{n}$ then when we write $\xi=\zeta+\left(\pi \hat{f}_{x}\right) \nu$ with $\zeta \in \mathcal{V}\left(b^{\prime}, b\right)$ and $\nu \in \mathcal{K}\left(b^{\prime}, b\right)$, then $t^{n}$ divides both $\zeta$ and $\nu$.

Proof. Write $\hat{f}(t, x)=x^{d}+\sum_{j=0}^{d-1} \hat{a}_{j} x^{j}+t x$. Now, with

$$
\pi \hat{f}_{x}(t, x)=\pi\left(d x^{d}+\sum_{j=1}^{d-1} j \hat{a}_{j} x^{j}+t x\right)
$$

we may write, for $m \geq d$,

$$
\begin{aligned}
x^{m} & =\pi d x^{d}\left(\frac{1}{\pi d} x^{m-d}\right)+\pi\left(\sum_{j=1}^{d-1} j \hat{a}_{j} x^{j}+t x\right)\left(\frac{1}{\pi d} x^{m-d}-\frac{1}{\pi d} x^{m-d}\right) \\
& =\pi \hat{f}_{x}(t, x)\left(\frac{1}{\pi d} x^{m-d}\right)-\sum_{j=1}^{d-1} \frac{j}{d} \hat{a}_{j} x^{m+j-d}-\frac{1}{d} t x^{m+1-d} .
\end{aligned}
$$

Notice that the last right-hand sum consists of terms in $x$ of degree strictly smaller than $x^{m}$. This is our reduction formula for $x^{m}$, reducing all monomials $x^{m}$ to some linear combination of $\left\{x, x^{2}, \ldots, x^{d-1}\right\}$.

Next, consider $B_{n m} t^{n} x^{m} \in \mathcal{K}\left(b^{\prime}, b ; 0\right)$ with $B_{n m} \in \mathbb{Z}_{q}[\pi]$. The reduction formula takes the form

$$
B_{n m} t^{n} x^{m}=\pi \hat{f}_{x}(t, x)\left(\frac{B_{n m}}{\pi d} t^{n} x^{m-d}\right)-\sum_{j=1}^{d-1} \frac{j B_{n m}}{d} \hat{a}_{j} t^{n} x^{m+j-d}-\frac{B_{n m}}{d} t^{n+1} x^{m+1-d} .
$$

Observe that, since $m \geq d$, it is immediate that $\frac{B_{n m}}{\pi d} t^{n} x^{m-d} \in \mathcal{K}\left(b^{\prime}, b ; e\right)$ while the other terms $\frac{j B_{n m}}{d} \hat{a}_{j} t^{n} x^{m+j-d}$ and $\frac{B_{n m}}{d} t^{n+1} x^{m+1-d}$ lie in $\mathcal{K}\left(b^{\prime}, b ; 0\right)$ since $b \geq b^{\prime}$. Iterating the recursive equation 16 , we obtain

$$
B_{n m} t^{n} x^{m} \in \mathcal{V}\left(b^{\prime}, b ; 0\right)+\pi \hat{f}_{x}(t, x) \mathcal{K}\left(b^{\prime}, b ; e\right) .
$$

Next, let $\xi=\sum_{n, m \geq 0} B_{n m} t^{n} x^{m} \in \mathcal{K}\left(b^{\prime}, b ; 0\right)$. For each $N \in \mathbb{Z}_{\geq 0}$ we may write $\xi=\zeta^{(N)}+\sum_{n \geq 0} \eta^{(n, N)}$ where

$$
\eta^{(n, N)}:=\sum_{m=0}^{N} B_{n m} t^{n} x^{m} \quad \text { and } \quad \zeta^{(N)}:=\sum_{n \geq 0} \sum_{m \geq N+1} B_{n m} t^{n} x^{m} .
$$

Observe that $x^{N+1} \mid \zeta^{(N)}$ for every $N$, and $t^{n} \mid \eta^{(n, N)}$. By 17 , we may write $\eta^{(n, N)}=\nu_{1}^{(n, N)}+\left(\pi \hat{f}_{x}\right) \nu_{2}^{(n, N)}$ with $\nu_{1}^{(n, N)} \in V\left(b^{\prime}, b ; 0\right)$ and $\nu_{2}^{(n, N)} \in \mathcal{K}\left(b^{\prime}, b ; e\right)$, both with the property that they are divisible by $t^{n}$. Hence, $\sum_{n \geq 0} \nu_{1}^{(n, N)}$ and $\sum_{n \geq 0} \nu_{2}^{(n, N)}$ produce well-defined elements of $V\left(b^{\prime}, b ; 0\right)$ and $\mathcal{K}\left(b^{\prime}, b ; e\right)$, respectively. Let us denote these elements by $\nu_{1}^{(N)}$ and $\nu_{2}^{(N)}$. We have thus constructed sequences of elements $\left\{\nu_{1}^{(N)}\right\}_{N \geq 1}$ in $V\left(b^{\prime}, b ; 0\right)$ and $\left\{\nu_{2}^{(N)}\right\}_{N \geq 1}$ in $\mathcal{K}\left(b^{\prime}, b ; e\right)$ which satisfy

$$
\xi=\zeta^{(N)}+\nu_{1}^{(N)}+\left(\pi \hat{f}_{x}\right) \nu_{2}^{(N)}
$$

Now, in the topology of coefficient-wise convergence (i.e. the $(\pi, t, x)$-adic topology), $\mathbb{Z}_{q}[\pi][[t, x]]$ is compact. Thus, $\mathcal{K}\left(b^{\prime}, b ; \rho\right)$ is compact for each $\rho$ in the induced topology. Hence, we may restrict ourselves to convergent subsequences of $\left\{\nu_{1}^{(N)}\right\}_{N \geq 1}$ and $\left\{\nu_{2}^{(N)}\right\}_{N \geq 1}$ with limits $\nu_{1} \in V\left(b^{\prime}, b ; 0\right)$ and $\nu_{2} \in \mathcal{K}\left(b^{\prime}, b ; e\right)$, respectively. Thus,

$$
\xi=\lim _{N \rightarrow \infty}\left(\zeta^{(N)}+\nu_{1}^{(N)}+\left(\pi \hat{f}_{x}\right) \nu_{2}^{(N)}\right)=\nu_{1}+\left(\pi \hat{f}_{x}\right) \nu_{2},
$$

where $\lim _{N \rightarrow \infty} \zeta^{(N)}=0$ since $x^{N+1} \mid \zeta^{(N)}$ for each $N$. This proves the lemma. 
Lemma 6.6. Let $b$ and $b^{\prime}$ be real numbers. Then $\mathcal{V}\left(b^{\prime}, b\right) \cap \pi \hat{f}_{x} \mathcal{K}\left(b^{\prime}, b\right)=\{0\}$.

Proof. Suppose $\eta:=c_{1} x+\cdots+c_{d-1} x^{d-1} \in \mathcal{V}\left(b^{\prime}, b\right) \cap \pi \hat{f}_{x} \mathcal{K}\left(b^{\prime}, b\right)$. Let $\zeta:=\sum_{j=0}^{\infty} B_{j} x^{j} \in \mathcal{K}\left(b^{\prime}, b\right)$ satisfy $\eta=\pi \hat{f}_{x} \zeta$. Now,

$$
\pi \hat{f}_{x} \sum_{j=0}^{\infty} B_{j} x^{j}=c_{1} x+c_{2} x^{2}+\cdots+c_{d-1} x^{d-1} .
$$

Writing $\pi \hat{f}_{x}(t, x)=\pi\left(d x^{d}+\sum_{j=1}^{d-1} j \hat{a}_{j} x^{j}+t x\right)$, we have

$$
\sum_{j=0}^{\infty} \pi d B_{j} x^{d+j}+\sum_{r=0}^{\infty} \sum_{j=1}^{d-1} \pi B_{r} \hat{a}_{j} x^{j+r}+\sum_{j=0}^{\infty} \pi B_{j} t x^{j=1}=c_{1} x+\cdots+c_{d-1} x^{d-1} .
$$

For $j \geq 0$, since the coefficient of $x^{d+j}$ in this equation must vanish, we have

$$
\pi d B_{j}+\sum_{r=j+1}^{d+j-2} \pi B_{r} \hat{a}_{d+j-r}+\pi B_{d+j-1} t=0
$$

and so

$$
B_{j}=-\frac{1}{d} \sum_{r=j+1}^{d+j-2} B_{r} \hat{a}_{d+j-r}-\frac{1}{d} B_{d+j-1} t .
$$

Using (18) recursively, we see that $B_{j} \rightarrow 0(p, t)$-adically. Hence, $B_{j}=0$ for all $j \geq 0$ as desired.

Lemma 6.7. Let $\xi \in \mathcal{K}\left(b^{\prime}, b\right)$ and suppose $\pi \hat{f}_{x} \xi \in \mathcal{K}\left(b^{\prime}, b ; \rho\right)$. Then $\xi \in \mathcal{K}\left(b^{\prime}, b ; \rho+e\right)$.

Proof. Let $\xi=\sum_{j=0}^{\infty} B_{j} x^{j} \in \mathcal{K}\left(b^{\prime}, b\right)$ and $\pi \hat{f}_{x} \xi=\sum_{j=0}^{\infty} C_{j} x^{j} \in \mathcal{K}\left(b^{\prime}, b ; \rho\right)$. Writing $\pi \hat{f}_{x}(t, x)=\pi\left(d x^{d}+\right.$ $\left.\sum_{j=1}^{d-1} j \hat{a}_{j}(t) x^{j}\right)$, where $\hat{a}_{1}(t):=t$, we have

$$
\sum_{j=0}^{\infty} \pi d B_{j} x^{d+j}+\sum_{r=0}^{\infty} \sum_{j=1}^{d-1} \pi B_{r} \hat{a}_{j} x^{j+r}=\sum_{j=0}^{\infty} C_{j} x^{j} .
$$

From this, the coefficient of $x^{d+j}$ satisfies

$$
\pi d B_{j}+\sum_{r=j+1}^{d+j-1} \pi B_{r} \hat{a}_{d+j-r}=C_{d+j}
$$

for all $j \geq 0$. Rewriting this, we have

$$
B_{j}=\frac{1}{\pi d} C_{d+j}-\frac{1}{d} \sum_{r=j+1}^{d+j-1} B_{r} \hat{a}_{d+j-r} .
$$

Iterating this $n$-times produces

$$
B_{j}=\zeta_{n}^{(j)}+\left(\xi_{1}^{(j)}+\xi_{2}^{(j)}+\cdots+\xi_{n}^{(j)}\right)
$$

where

$$
\zeta_{n}^{(j)}:= \pm \sum_{r_{1}=j+1}^{d+j-1} \sum_{r_{2}=r_{1}+1}^{d+r_{1}-1} \cdots \sum_{r_{n}=r_{n-1}+1}^{d+r_{n-1}-1} \frac{1}{d^{n}} B_{r_{n}} \hat{a}_{d+j-r_{1}} \hat{a}_{d+r_{1}-r_{2}} \cdots \hat{a}_{d+r_{n-1}-r_{n}}
$$

and

$$
\begin{aligned}
\xi_{1}^{(j)} & :=\frac{1}{\pi d} C_{d+j} \\
\xi_{2}^{(j)} & :=\frac{1}{d^{2} \pi} \sum_{r_{1}=j+1}^{d+j-1} C_{d+r_{1}} \hat{a}_{d+j-r_{1}} \\
\xi_{3}^{(j)} & :=\frac{1}{d^{3} \pi} \sum_{r_{1}=j+1}^{d+j-1} \sum_{r_{2}=r_{1}+1}^{d+r_{1}-1} C_{d+r_{2}} \hat{a}_{d+j-r_{1}} \hat{a}_{d+r_{1}-r_{2}} \\
& : \\
\xi_{n}^{(j)} & :=\frac{1}{d^{n} \pi} \sum_{r_{1}=j+1}^{d+j-1} \sum_{r_{2}=r_{1}+1}^{d+r_{1}-1} \cdots \sum_{r_{n-1}=r_{n-2}+1}^{d+r_{n-2}-1} C_{d+r_{n-1}} \hat{a}_{d+j-r_{1}} \hat{a}_{d+r_{1}-r_{2}} \cdots \hat{a}_{d+r_{n-2}-r_{n-1}} .
\end{aligned}
$$


Since $B_{r_{n}} \in L\left(b^{\prime} ; b w_{0}\left(r_{n}\right)\right), \zeta_{n}^{(j)} \rightarrow 0$ as $n$ tends to infinity. Thus, to complete the lemma, let us show $\sum_{n=1}^{\infty} \xi_{n}^{(j)} \in$ $L\left(b^{\prime} ; b w_{0}(j)+e\right)$.

We know $C_{d+r_{n-1}} \in L\left(b^{\prime} ; b w_{0}\left(d+r_{n-1}\right)+\rho\right)$. We wish to show

$$
\frac{1}{d^{n-1} \pi} C_{d+r_{n-1}} \hat{a}_{d+j-r_{1}} \hat{a}_{d+r_{1}-r_{2}} \cdots \hat{a}_{d+r_{n-2}-r_{n-1}} \in L\left(b^{\prime} ; b w_{0}(j)+e+\rho\right) .
$$

Notice that 19 typically has many $\hat{a}$ terms equal to 1 . These $\hat{a}$ will not affect the $L\left(b^{\prime} ; \sigma\right)$ space that $19 p$ lies in. It is only when the $\hat{a}$ equals $t$ that things change. The worse case is when all $\hat{a}$ equal $t$. In this case, $r_{i}=i d+j-i$ for $i=1,2, \ldots, n-1$ making 19 take the form

$$
\frac{1}{d^{n-1} \pi} C_{d+(n-1) d+j-(n-1)} t^{n-1}
$$

which may easily be shown to lie in $L\left(b^{\prime} ; b w_{0}(j)+e+\rho\right)$. The general case is similar. This concludes the proof of the lemma.

Lemma 6.8. Let $b^{\prime} \leq b$ and $\frac{1}{p-1} \leq b \leq \frac{p}{p-1}$. With $e:=b-\frac{1}{p-1}$ we have

$$
\mathcal{K}\left(b^{\prime}, b ; 0\right)^{\bullet} \subset \mathcal{V}\left(b^{\prime}, b ; 0\right)+D(t) \mathcal{K}\left(b^{\prime}, b ; e\right)
$$

Furthermore, if $\xi \in \mathcal{K}\left(b^{\prime}, b\right)^{\bullet}$ is divisible by $t^{n}$, then when we write $\xi=\eta+D(t) \zeta$ with $\eta \in \mathcal{V}\left(b^{\prime}, b\right)$ and $\zeta \in \mathcal{K}\left(b^{\prime}, b\right)$, then $\eta$ and $\zeta$ are also divisible by $t^{n}$.

Proof. Recall, $D(t)=x \frac{\partial}{\partial x}+H(t, x)$ with $H(t, x):=\sum_{j=0}^{\infty} \pi_{j} p^{j} \hat{f}_{x}^{\tau^{j}}\left(t^{p^{j}}, x^{p^{j}}\right)$. Now, observe that

$$
\hat{f}_{x}^{\tau^{j}}\left(t^{p^{j}}, x^{p^{j}}\right)=\hat{f}_{x}(t, x)^{p^{j}}+p h_{j}(t, x)
$$

for some polynomial $h_{j}$ with coefficients in $\mathbb{Z}_{q}[\pi]$. Write

$$
H(t, x)=\pi \hat{f}_{x}(t, x) Q_{1}(t, x)+K_{1}(t, x)
$$

where

$$
\begin{aligned}
Q_{1}(t, x) & :=\sum_{j=0}^{\infty} \pi_{j} \pi^{-1} p^{j} \hat{f}_{x}(t, x)^{p^{j}-1} \\
K_{1}(t, x) & :=\sum_{j=1}^{\infty} \pi_{j} p^{j+1} h_{j}(t, x) .
\end{aligned}
$$

We claim that $Q_{1}, \frac{1}{Q_{1}}, K_{1} \in \mathcal{K}\left(\frac{p}{p-1}, \frac{p}{p-1} ; 0\right)$. To see this, note that since $\hat{f} \in \mathcal{K}\left(\frac{p}{p-1}, \frac{p}{p-1} ;-\frac{p}{p-1}\right)$, we have

$$
\hat{f}_{x}^{p^{j}-1} \in \mathcal{K}\left(\frac{p}{p-1}, \frac{p}{p-1} ;-\left(\frac{p}{p-1}\right)\left(p^{j}-1\right)\right)
$$

Thus,

$$
\pi_{j} \pi^{-1} p^{j} \hat{f}_{x}^{p^{j}-1} \in \mathcal{K}\left(\frac{p}{p-1}, \frac{p}{p-1} ; 0\right)
$$

proving the result for $Q_{1}$ and $1 / Q_{1}$. Next, since $h_{j}$ consists of terms coming from the expansion of $\hat{f}_{x}^{p^{j}}$, we see that $h_{j} \in \mathcal{K}\left(\frac{p}{p-1}, \frac{p}{p-1} ;-\left(\frac{p}{p-1}\right) p^{j}\right)$. Thus $\pi_{j} p^{j+1} h_{j} \in \mathcal{K}\left(\frac{p}{p-1}, \frac{p}{p-1} ; 0\right)$ proving the result for $K_{1}$.

We will first suppose $b>\frac{1}{p-1}$. Let $\xi \in \mathcal{K}\left(b^{\prime}, b ; 0\right)^{\bullet}$. By Lemma 6.5 , there exists $\eta_{1} \in \mathcal{V}\left(b^{\prime}, b ; 0\right)$ and $\zeta_{1} \in$ $\mathcal{K}\left(b^{\prime}, b ; e\right)$ such that $\xi=\eta_{1}+\left(\pi \hat{f}_{x}\right) \zeta_{1}$. Thus,

$$
\begin{aligned}
\xi & =\eta_{1}+\left(H-K_{1}\right) Q_{1}^{-1} \zeta_{1} \\
& =\eta_{1}+(\underbrace{Q_{1}^{-1} K_{1} \zeta_{1}-x \frac{\partial}{\partial x} Q_{1}^{-1} \zeta_{1}}_{=: \nu_{1}})+D(t)(\underbrace{Q_{1}^{-1} \zeta_{1}}_{=: \zeta_{1}^{\prime}}) .
\end{aligned}
$$

Notice that $\nu_{1} \in \mathcal{K}\left(b^{\prime}, b ; e\right)^{\bullet}$ and $\zeta_{1}^{\prime} \in \mathcal{K}\left(b^{\prime}, b ; e\right)$. Continuing this same process, but now with $\nu_{1}$ instead of $\xi$, we are lead to

$$
\xi=\left(\eta_{1}+\cdots+\eta_{N}\right)+\nu_{N}+D(t)\left(\zeta_{1}^{\prime}+\cdots+\zeta_{N}^{\prime}\right)
$$


where $\eta_{i} \in \mathcal{V}\left(b^{\prime}, b ;(i-1) e\right), \nu_{N} \in \mathcal{K}\left(b^{\prime}, b ; N e\right)^{\bullet}$, and $\zeta_{i}^{\prime} \in \mathcal{K}\left(b^{\prime}, b ; i e\right)$. Thus, $\eta:=\sum_{i=1}^{\infty} \eta_{i} \in \mathcal{V}\left(b^{\prime}, b ; 0\right)$ and $\zeta^{\prime}:=\sum_{i=1}^{\infty} \zeta_{i}^{\prime} \in \mathcal{K}\left(b^{\prime}, b ; e\right)$. Upon taking the limit in the coefficientwise convergence topology we see that

$$
\xi=\eta+D(t) \zeta^{\prime}
$$

as desired.

We now consider the case when $b=\frac{1}{p-1}$. Let $\xi \in \mathcal{K}\left(b^{\prime}, b ; 0\right)^{\bullet}$. For each $N \in \mathbb{Z}_{\geq 0}$, we may write

$$
\xi=\sum_{n=1}^{N} B_{n} x^{n}+\sum_{n \geq N+1} B_{n} x^{n} .
$$

Let $\epsilon>0$. For $1 \leq n \leq N$, since $b w_{0}(n) \geq\left(b+\frac{\epsilon}{w_{0}(N)}\right) w_{0}(n)-\epsilon$ we see that

$$
\sum_{n=1}^{N} B_{n} x^{n} \in \mathcal{K}\left(b^{\prime}, b+\frac{\epsilon}{w_{0}(N)} ;-\epsilon\right)^{\bullet} .
$$

Since $b+\frac{\epsilon}{w_{0}(N)}>\frac{1}{p-1}$, there exists $\eta^{(\epsilon, N)} \in \mathcal{V}\left(b^{\prime}, b+\frac{\epsilon}{w_{0}(N)} ;-\epsilon\right)$ and $\zeta^{(\epsilon, N)} \in \mathcal{K}\left(b^{\prime}, b+\frac{\epsilon}{w_{0}(N)} ;-\epsilon+\frac{\epsilon}{w_{0}(N)}\right)$ such that

$$
\sum_{n=1}^{N} B_{n} x^{n}=\eta^{(\epsilon, N)}+D(t) \zeta^{(\epsilon, N)}
$$

We have just constructed sequences $\left\{\eta^{(\epsilon, N)}\right\}_{N=1}^{\infty} \subset \mathcal{V}\left(b^{\prime}, b ;-\epsilon\right)$ and $\left\{\zeta^{(\epsilon, N)}\right\}_{N=1}^{\infty} \subset \mathcal{K}\left(b^{\prime}, b ;-\epsilon\right)$. Since $\mathcal{K}\left(b^{\prime}, b ; \rho\right)$ and $\mathcal{V}\left(b^{\prime}, b ; \rho\right)$ are compact in the coefficientwise convergence topology for each $\rho$, we may restrict ourselves to convergent subsequences with limits $\eta^{(\epsilon)} \in \mathcal{V}\left(b^{\prime}, b ;-\epsilon\right)$ and $\zeta^{(\epsilon)} \in \mathcal{K}\left(b^{\prime}, b ;-\epsilon\right)$ which satisfy

$$
\xi=\eta^{(\epsilon)}+D(t) \zeta^{(\epsilon)}
$$

In the coefficientwise convergence topology, letting $\epsilon \rightarrow 0^{+}$, there exists $\eta \in \mathcal{V}\left(b^{\prime}, b ; 0\right)$ and $\zeta \in \mathcal{K}\left(b^{\prime}, b ; 0\right)$ such that

$$
\xi=\lim _{\epsilon \rightarrow 0^{+}} \eta^{(\epsilon)}+D(t) \zeta^{(\epsilon)}=\eta+D(t) \zeta
$$

This proves the first part of the lemma.

The second part follows from the divisibility result in Lemma 6.5 and running through the above argument.

Lemma 6.9. Let $b^{\prime} \leq b$ and $\frac{1}{p-1} \leq b \leq \frac{p}{p-1}$. Then

$$
\mathcal{V}\left(b^{\prime}, b\right) \cap D(t) \mathcal{K}\left(b^{\prime}, b\right) .
$$

Proof. Let us first assume $b>\frac{1}{p-1}$. Let $\eta \in V\left(b^{\prime}, b\right) \cap D(t) \mathcal{K}\left(b^{\prime}, b\right)$ be non-zero, and let $\xi \in \mathcal{K}\left(b^{\prime}, b\right)$ be such that $D(t) \xi=\eta$. Find a real number $c$ such that $\xi \in \mathcal{K}\left(b^{\prime}, b ; c\right)$ but $\xi \notin \mathcal{K}\left(b^{\prime}, b ; c+e\right)$. We will prove that no such $c$ exists, contradicting the existence of $\eta$.

Since $D(t)=x \frac{\partial}{\partial x}+\left(\pi \hat{f}_{x}\right) Q_{1}+K_{1}$, we have

$$
\eta=D(t) \xi=\left(\pi \hat{f}_{x}\right) Q_{1} \xi+\left(x \frac{\partial}{\partial x} \xi+K_{1} \xi\right) .
$$

Since $x \frac{\partial}{\partial x} \xi+K_{1} \xi \in \mathcal{K}\left(b^{\prime}, b ; c\right)^{\bullet}$, by Lemma 6.8 there exists $\nu_{1} \in \mathcal{V}\left(b^{\prime}, b ; c\right)$ and $\zeta_{1} \in \mathcal{K}\left(b^{\prime}, b ; c+e\right)$ such that

$$
x \frac{\partial}{\partial x} \xi+K_{1} \xi=\nu_{1}+D(t) \zeta_{1}
$$

Hence,

$$
\begin{aligned}
\eta & =\left(\pi \hat{f}_{x}\right) Q_{1} \xi+\nu_{1}+D(t) \zeta_{1} \\
& =\left(\pi \hat{f}_{x}\right) Q_{1}\left(\xi+\zeta_{1}\right)+\nu_{1}+K_{1} \zeta_{1} .
\end{aligned}
$$

Since $K_{1} \zeta_{1} \in \mathcal{K}\left(b^{\prime}, b ; c+e\right)$, applying Lemma 6.8 again produces $\nu_{2} \in \mathcal{V}\left(b^{\prime}, b ; c+e\right)$ and $\zeta_{2} \in \mathcal{K}\left(b^{\prime}, b ; c+2 e\right)$ such that $K_{1} \zeta_{1}=\nu_{2}+D(t) \zeta_{2}$. Thus,

$$
\eta=\left(\pi \hat{f}_{x}\right) Q_{1}\left(\xi+\zeta_{1}+\zeta_{2}\right)+\left(\nu_{1}+\nu_{2}\right)+K_{1} \zeta_{2} .
$$


Iterating this via induction, and taking the limit in the coefficient-wise convergence topology, we obtain

$$
\eta=\left(\pi \hat{f}_{x}\right) Q_{1}\left(\xi+\sum_{i=1}^{\infty} \zeta_{i}\right)+\sum_{i=1}^{\infty} \nu_{i} .
$$

This means $\left(\pi \hat{f}_{x}\right) Q_{1}\left(\xi+\sum_{i=1}^{\infty} \zeta_{i}\right) \in \mathcal{V}\left(b^{\prime}, b\right)$, and so by Lemma 6.6. $\xi=-\sum_{i=1}^{\infty} \zeta_{i} \in \mathcal{K}\left(b^{\prime}, b ; c\right)$ which is impossible.

Suppose now that $b=\frac{1}{p-1}$ and $\eta \in \mathcal{V}\left(b^{\prime}, b\right) \cap D(t) \mathcal{K}\left(b^{\prime}, b\right)$. With this choice of $b, \alpha_{1}:=\tau^{-1} \circ \psi_{x} \circ F(t, x)$ is a map from $\mathcal{K}\left(b^{\prime}, b\right)$ to $\mathcal{K}\left(b^{\prime}, p b\right)$. Therefore, since $\alpha_{1} \circ D(t)=p D\left(t^{p}\right) \circ \alpha_{1}$, we see that

$$
\alpha_{1}(\eta) \in D\left(t^{p}\right) \mathcal{K}\left(b^{\prime}, p b\right)
$$

Thus, the reduction of $\alpha_{1}(\eta)$ equals zero in $\mathcal{H}_{1, t^{p}}\left(b^{\prime}, p b\right)$. Now, we may also view $\alpha_{1}$ as an endomorphism of $\mathcal{K}\left(b^{\prime}, p b\right)$ and so, abusing notation, we obtain a map on homology $\bar{\alpha}_{1}: \mathcal{H}_{1, t}\left(b^{\prime}, p b\right) \rightarrow \mathcal{H}_{1, t^{p}}\left(b^{\prime}, p b\right)$. By Lemma 6.10 below, this map $\bar{\alpha}_{1}$ is invertible. Therefore, since the reduction of $\alpha_{1}(\eta)$ is zero in $\mathcal{H}_{1, t^{p}}\left(b^{\prime}, p b\right)$, we must have the reduction of $\eta$ in $\mathcal{H}_{1, t}\left(b^{\prime}, p b\right)$ equal to zero as well. Hence, $\eta \in D(t) \mathcal{K}\left(b^{\prime}, p b\right)$. Since $D(t) \mathcal{K}\left(b^{\prime}, p b\right) \subset \mathcal{K}\left(b^{\prime}, p b\right)$, we see that $\eta \in V\left(b^{\prime}, p b\right) \cap D(t) \mathcal{K}\left(b^{\prime}, p b\right)$. However, this intersection equals $\{0\}$ by the argument above since $b p>1 /(p-1)$, proving $\eta=0$ as desired.

Lemma 6.10. Let $b^{\prime} \leq 1 /(p-1)$. Then $\bar{\alpha}_{1}: \mathcal{H}_{1, t}\left(b^{\prime}, p /(p-1)\right) \rightarrow \mathcal{H}_{1, t^{p}}\left(b^{\prime}, p /(p-1)\right)$ is an isomorphism.

Proof. Since $b^{\prime} \leq 1 /(p-1)$, it follows from definition that $\alpha_{1}$ is a map from $\mathcal{K}\left(b^{\prime}, 1 /(p-1)\right)$ to $\mathcal{K}\left(b^{\prime}, p /(p-1)\right)$. Now, define a map $\alpha_{1}^{\prime}: \mathcal{K}\left(b^{\prime}, p /(p-1)\right) \rightarrow \mathcal{K}\left(b^{\prime}, 1 /(p-1)\right)$ by

$$
\alpha_{1}^{\prime}:=F(t, x)^{-1} \circ \Phi_{x} \circ \tau
$$

where $\Phi_{x}$ is the map $x \mapsto x^{p}$. Clearly, $\alpha_{1} \circ \alpha_{1}^{\prime}=i d$, the identity map on $\mathcal{K}\left(b^{\prime}, p /(p-1)\right)$. Hence, we have

$$
\mathcal{K}\left(b^{\prime}, p /(p-1)\right)=\alpha_{1} \alpha_{1}^{\prime} \mathcal{K}\left(b^{\prime}, p /(p-1)\right) \subset \alpha_{1} \mathcal{K}\left(b^{\prime}, 1 /(p-1)\right) \subset \mathcal{K}\left(b^{\prime}, p /(p-1)\right) .
$$

Hence, $\alpha_{1}$ maps $\mathcal{K}\left(b^{\prime}, 1 /(p-1)\right)$ isomorphically onto $\mathcal{K}\left(b^{\prime}, p /(p-1)\right)$. A similar argument shows $\alpha_{1}$ maps $\mathcal{K}\left(b^{\prime}, 1 /(p-1)\right)^{\bullet}$ isomorphically onto $\mathcal{K}\left(b^{\prime}, p /(p-1)\right)^{\bullet}$.

By Lemma 6.8, we know

$$
\mathcal{K}\left(b^{\prime}, 1 /(p-1)\right)^{\bullet} \subset \mathcal{V}\left(b^{\prime}, 1 /(p-1)\right)+D(t) \mathcal{K}\left(b^{\prime}, 1 /(p-1)\right) .
$$

Applying $\alpha_{1}$ to this we obtain

$$
\mathcal{K}\left(b^{\prime}, p /(p-1)\right)^{\bullet}=\alpha_{1} \mathcal{K}\left(b^{\prime}, 1 /(p-1)\right) \subset \alpha_{1} \mathcal{V}\left(b^{\prime}, 1 /(p-1)\right)+D\left(t^{p}\right) \mathcal{K}\left(b^{\prime}, p /(p-1)\right) .
$$

Since $\mathcal{V}\left(b^{\prime}, p /(p-1)\right) \subset \mathcal{K}\left(b^{\prime}, p /(p-1)\right)^{\bullet}$, we have

$$
\mathcal{V}\left(b^{\prime}, p /(p-1)\right) \subset \alpha_{1} \mathcal{V}\left(b^{\prime}, 1 /(p-1)\right)+D\left(t^{p}\right) \mathcal{K}\left(b^{\prime}, p /(p-1)\right) .
$$

Now, it follows from the definition that $\mathcal{V}\left(b^{\prime}, b_{1}\right)=\mathcal{V}\left(b^{\prime}, b_{2}\right)$ for any positive real numbers $b_{1}$ and $b_{2}$. Thus,

$$
\mathcal{V}\left(b^{\prime}, p /(p-1)\right) \subset \alpha_{1} \mathcal{V}\left(b^{\prime}, p /(p-1)\right)+D\left(t^{p}\right) \mathcal{K}\left(b^{\prime}, p /(p-1)\right) .
$$

Viewing $\alpha_{1}$ as an endomorphism of $\mathcal{K}\left(b^{\prime}, p /(p-1)\right)^{\bullet}$, this shows $\bar{\alpha}_{1}: \mathcal{H}_{1, t}\left(b^{\prime}, p /(p-1)\right) \rightarrow \mathcal{H}_{1, t^{p}}\left(b^{\prime}, p /(p-1)\right)$ is surjective. Since both of these spaces are free $L\left(b^{\prime}\right)$-modules of finite rank, $\bar{\alpha}_{1}$ must also be injective. This finishes the lemma.

Lemma 6.11. Suppose $b>\frac{1}{p-1}$. If $\xi \in \mathcal{K}\left(b^{\prime}, b\right)$ and $D(t) \xi \in \mathcal{K}\left(b^{\prime}, b ; \rho\right)$, then $\xi \in \mathcal{K}\left(b^{\prime}, b ; \rho+e\right)$.

Proof. Suppose $\xi \neq 0$. Choose $c \in \mathbb{R}$ such that $\xi \in \mathcal{K}\left(b^{\prime}, b ; c\right)$ but $\xi \notin \mathcal{K}\left(b^{\prime}, b ; c+e\right)$. Then

$$
\left(\pi \hat{f}_{x}\right) Q_{1} \xi=D(t) \xi-x \frac{\partial}{\partial x} \xi-K_{1} \xi \in \mathcal{K}\left(b^{\prime}, b ; \min \{\rho, c\}\right)
$$

Thus, $\left(\pi \hat{f}_{x}\right) \xi \in \mathcal{K}\left(b^{\prime}, b ; \min \{\rho, c\}\right)$ which, by Lemma 6.7 . implies $\xi \in \mathcal{K}\left(b^{\prime}, b ; \min \{\rho, c\}+e\right)$. By our choice of $c$ the lemma follows.

Corollary 6.12. Suppose $b>\frac{1}{p-1}$. Then $D(t)$ is injective.

Proof. Suppose there exists nonzero $\xi \in \mathcal{K}\left(b^{\prime}, b\right)$ such that $D(t) \xi=0$. Then, by Lemma 6.11, since $0 \in \mathcal{K}\left(b^{\prime}, b ; \rho\right)$ for every $\rho$, we have $\xi \in \mathcal{K}\left(b^{\prime}, b ; \rho+e\right)$ for every $\rho$. Hence, $\xi$ must be zero. 


\subsection{Dwork decomposition for the symmetric powers of relative homology}

Theorem 6.1 demonstrated one consequence of Dwork decomposition with the operator $\partial$. In this section, we wish to take the hypothesis of Dwork decomposition in that theorem and reduce it to a similar hypothesis which replaces the differential operator $\partial$ with an easier operator $\mathcal{L}_{\Phi}$ described below. We expect that this similar hypothesis may be demonstrated for a large class of $f(t, x)$. However, we also expect that it will fail just as often. New ideas will be required to handle the latter case.

It is easiest to see the main obstructions to the theory if we generalize a bit. Let $d$ be a positive integer. We call a function $w: \mathbb{Z}_{\geq 0}^{s} \rightarrow \frac{1}{d} \mathbb{Z}_{\geq 0}$ a weight function if it satisfies the following three properties:

1. $w(0)=0$,

2. $w(c u)=c w(u)$ for every $c \in \mathbb{Q}_{\geq 0}$, and

3. $w(u+v) \leq w(u)+w(v)$ for all $u, v \in \mathbb{Z}_{\geq 0}^{s}$.

Let $w_{1}: \mathbb{Z}_{\geq 0}^{s} \rightarrow \frac{1}{d_{1}} \mathbb{Z}_{\geq 0}$ be a weight function. Let $b$ and $b^{\prime}$ be positive real numbers. For each $\rho \in \mathbb{R}$ define

$$
L\left(b^{\prime} ; \rho\right):=\left\{\sum_{\mathbf{j} \in \mathbb{Z}_{\geq 0}^{s}} A_{\mathbf{j}} t^{\mathbf{j}} \mid A_{\mathbf{j}} \in \mathbb{Z}_{q}[\pi], \operatorname{ord}_{p}\left(A_{\mathbf{j}}\right) \geq b^{\prime} w_{1}(\mathbf{j})+\rho\right\} .
$$

This is a $p$-adic Banach space with norm given by $\left\|\sum A_{\mathbf{j}} \mathrm{t}^{\mathbf{j}}\right\|:=\min _{\mathbf{j}} \operatorname{ord}_{p}\left(A_{\mathbf{j}}\right)$. Define

$$
L\left(b^{\prime}\right):=\bigcup_{\rho \in \mathbb{R}} L\left(b^{\prime} ; \rho\right) .
$$

Let $\mathcal{M}$ be a free $L\left(b^{\prime}\right)$-module with basis $\left\{e_{1}, \ldots, e_{r}\right\}$. We place a weight on each basis element $e_{i}$ as follows: fix a positive integer $d_{0}$ and let $w_{0}:\{1, \ldots, r\} \rightarrow \frac{1}{d_{0}} \mathbb{Z}_{\geq 0}$ be any function. Note, $w_{0}$ is not a weight function since the set $\{1, \ldots, r\}$ is finite. Define

$$
\mathcal{M}\left(b^{\prime}, b ; \rho\right):=\left\{\sum_{i=1}^{r} B_{i} e_{i} \mid B_{i} \in L\left(b^{\prime} ; b w_{0}(i)+\rho\right)\right\}
$$

and

$$
\mathcal{M}\left(b^{\prime}, b\right):=\mathcal{M}=\bigcup_{\rho \in \mathbb{R}} \mathcal{M}\left(b^{\prime}, b ; \rho\right) .
$$

For any subset $U$ of $\mathcal{M}$, we may define $U\left(b^{\prime}, b ; \rho\right):=U \cap \mathcal{M}\left(b^{\prime}, b ; \rho\right)$ and $U\left(b^{\prime}, b\right):=U \cap \mathcal{M}\left(b^{\prime}, b\right)$.

Denote by $\mathcal{M}^{(k)}:=S y m^{k} \mathcal{M}$ the $k$-th symmetric power of $\mathcal{M}$ over $L\left(b^{\prime}\right)$. Similar to 20 define

$$
\mathcal{M}^{(k)}\left(b^{\prime}, b ; \rho\right):=\left\{\sum_{\substack{\mathbf{i}:=\left(i_{1}, \ldots, i_{r}\right) \in \mathbb{Z}_{\geq 0}^{r} \\ i_{1}+\cdots+i_{r}=k}} B_{\mathbf{i}} e^{\mathbf{i}} \mid B_{\mathbf{i}} \in L\left(b^{\prime} ; b w_{0}(\mathbf{i})+\rho\right)\right\}
$$

where $e^{\mathbf{i}}:=e_{1}^{i_{1}} \cdots e_{r}^{i_{r}}$ and

$$
w_{0}(\mathbf{i}):=w_{0}\left(i_{1}, \ldots, i_{r}\right):=\sum_{j=1}^{r} i_{j} w_{0}(j) .
$$

Let $\mathcal{N}$ be a free, finite rank $L\left(b^{\prime}\right)$-module, and denote by $\mathcal{N}^{(k)}$ the $k$-th symmetric power of $\mathcal{N}$ over $L\left(b^{\prime}\right)$. Let $\Phi: \mathcal{M} \rightarrow \mathcal{N}$ be an $L\left(b^{\prime}\right)$-module morphism. Define the Leibnitz operator of $\Phi$ to be the operator $\mathcal{L}_{\Phi}: \mathcal{M}^{(k)} \rightarrow$ $\mathcal{N}^{(k)}$ defined by

$$
\mathcal{L}_{\Phi}\left(e_{1}^{i_{1}} \cdots e_{r}^{i_{r}}\right):=\sum_{m=1}^{r} i_{m} e_{1}^{i_{1}} \cdots e_{m}^{i_{m}-1} \cdots e_{r}^{i_{r}} \Phi\left(e_{m}\right) .
$$

Next, we mention a short technical lemma which will be useful.

Lemma 6.13. Suppose $\Phi: \mathcal{M}\left(b^{\prime}, b ; 0\right) \rightarrow \mathcal{N}\left(b^{\prime}, b ; \rho\right)$. Then $\mathcal{L}_{\Phi}: \mathcal{M}^{(k)}\left(b^{\prime}, b ; 0\right) \rightarrow \mathcal{N}^{(k)}\left(b^{\prime}, b ; \rho\right)$. 
Proof. Since $e_{i} \in \mathcal{M}\left(b^{\prime}, b ;-b w_{0}(i)\right)$ we have $\Phi\left(e_{i}\right)=a_{i, 1} e_{1}+\cdots+a_{i, r} e_{r} \in \mathcal{N}\left(b^{\prime}, b ; \rho-b w_{0}(i)\right)$. Thus, $a_{i, j} \in$ $L\left(b^{\prime} ; \rho+b w_{0}(j)-b w_{0}(i)\right)$. Similarly, since $e_{1}^{i_{1}} \cdots e_{r}^{i_{r}} \in \mathcal{M}\left(b^{\prime}, b ;-b w_{0}\left(i_{1}, \ldots, i_{r}\right)\right)$ we see that

$$
\begin{aligned}
& \mathcal{L}_{\Phi}\left(e_{1}^{i_{1}} \cdots e_{r}^{i_{r}}\right)=\sum_{l=1}^{r} i_{l} e_{1}^{i_{1}} \cdots e_{l}^{i_{l}-1} \cdots e_{r}^{i_{r}} \Phi\left(e_{i_{l}}\right) \\
&=\sum_{l=1}^{r}\left(\sum_{j<l} i_{l} a_{l, j} e_{1}^{i_{1}} \cdots e_{j}^{i_{j}+1} \cdots e_{l}^{i_{l}-1} \cdots e_{r}^{i_{r}}\right. \\
&+\sum_{j>l} i_{l} a_{l, j} e_{1}^{i_{1}} \cdots e_{j}^{i_{j}-1} \cdots e_{l}^{i_{l}+1} \cdots e_{r}^{i_{r}} \\
&\left.+i_{l} a_{l, l} e_{1}^{i_{1}} \cdots e_{r}^{i_{r}}\right) .
\end{aligned}
$$

Let us consider one of these terms, say $i_{l} a_{l, j} e_{1}^{i_{1}} \cdots e_{j}^{i_{j}+1} \cdots e_{l}^{i_{l}-1} \cdots e_{r}^{i_{r}}$ with $j<l$. Observe

$$
\begin{aligned}
\operatorname{ord}_{p}\left(a_{l, j}\right) & \geq \rho+b w_{0}(j)-b w_{0}(l) \\
& =\rho+b w_{0}\left(i_{1}, \ldots, i_{j}+1, \ldots, i_{l}-1, \ldots, i_{r}\right)-b w_{0}\left(i_{1}, \ldots, i_{r}\right) .
\end{aligned}
$$

The other terms are similar, proving $\mathcal{L}_{\Phi}\left(e^{\mathbf{i}}\right) \in \mathcal{N}^{(k)}\left(b^{\prime}, b ; \rho-b w_{0}(\mathbf{i})\right)$ as desired.

Let us now return to our particular family. With $\hat{f}_{t}:=t \frac{\partial}{\partial t} \hat{f}(t, x)$, since $\pi \hat{f}_{t} \in \mathcal{K}\left(\frac{p}{p-1}, \frac{p}{p-1} ;-1\right)$, by Theorem 6.4 we have for each $i=1, \ldots, d-1$,

$$
\pi \hat{f}_{t}(t, x) x^{i}=A_{i, 1} x+\cdots+A_{i, d-1} x^{d-1}+D(t)\left(h_{i}(t, x)\right)
$$

for some $A_{i, j} \in L\left(\frac{p}{p-1}\right)$ and $h_{i} \in \mathcal{K}\left(\frac{p}{p-1}, \frac{p}{p-1}\right)$. This defines a morphism

$$
\pi \hat{f}_{t}: \mathcal{H}_{1, t}\left(\frac{p}{p-1}, \frac{p}{p-1} ; 0\right) \rightarrow \mathcal{H}_{1, t}\left(\frac{p}{p-1}, \frac{p}{p-1} ;-1\right)
$$

via the matrix $\left(A_{i, j}\right)_{1 \leq i, j \leq d-1}$ which acts on the right. With $b^{\prime} \leq b$ and $b \leq p /(p-1)$, this defines an endomorphism $\mathcal{L}_{\pi \hat{f}_{t}}$ on $\mathcal{H}_{1, t}^{(k)}\left(b^{\prime}, b\right)$. Set $\mathcal{N}\left(b^{\prime}, b\right):=t \mathcal{H}_{1, t}^{(k)}\left(b^{\prime}, b\right)$ and $\mathcal{M}\left(b^{\prime}, b\right):=\mathcal{H}_{1, t}^{(k)}\left(b^{\prime}, b\right)$.

Theorem 6.14. Let $p$ be a prime number such that $(p, d)=1$. Suppose $\mathcal{L}_{\pi \hat{f}_{t}}$ satisfies the following: there exists a free, finite rank $\mathbb{Z}_{q}[\pi]$-submodule $V_{k}$ of $\mathcal{N}(p /(p-1), p /(p-1))$ such that for any $\frac{p}{p-1} \geq b \geq b^{\prime} \geq \frac{1}{p-1}$, with $e^{\prime}:=b^{\prime}-\frac{1}{p-1}$,

$$
\begin{aligned}
& \text { 1. } \mathcal{N}\left(b^{\prime}, b ; 0\right) \subset V_{k}\left(b^{\prime}, b ; 0\right)+\mathcal{L}_{\pi \hat{f}_{t}} \mathcal{M}\left(b^{\prime}, b ; e^{\prime}\right) \\
& \text { 2. } V_{k}\left(b^{\prime}, b\right) \cap \mathcal{L}_{\pi \hat{f}_{t}} \mathcal{M}\left(b^{\prime}, b\right)=\{0\}
\end{aligned}
$$

Then $\partial$ satisfies:

1. $\mathcal{N}\left(b^{\prime}, b ; 0\right) \subset V_{k}\left(b^{\prime}, b ; 0\right)+\partial \mathcal{M}\left(b^{\prime}, b ; e^{\prime}\right)$

2. $V_{k}\left(b^{\prime}, b\right) \cap \partial \mathcal{M}\left(b^{\prime}, b\right)=\{0\}$ for $\frac{1}{p-1}<b^{\prime} \leq \frac{p}{p-1}$ and $\frac{1}{p-1} \leq b \leq \frac{p}{p-1}$.

As an example of the theorem, consider the cubic family $f(t, x)=x^{3}+t x$. In [15] it was shown that $\mathcal{L}_{\pi \hat{f}_{t}}$ satisfied the hypothesis in the theorem when $k$ is odd and $k<p$. Consequently, we have the following result.

Corollary 6.15. Let $f(t, x)=x^{3}+t x$ and $p \geq 5$ be a prime number. Suppose $k$ is an odd positive integer satisfying $k<p$. Let $V_{k}$ denote the $\mathbb{Z}_{q}[\pi]$-span of the set $\left\{t e_{1}^{k-2 i} e_{2}^{2 i}\right\}_{i=0}^{\frac{k-1}{2}} \subset \mathcal{N}$ Then

$$
\mathcal{N}\left(b^{\prime}, b ; 0\right) \subset V_{k}\left(b^{\prime}, b ; 0\right)+\partial \mathcal{M}\left(b^{\prime}, b ; e^{\prime}\right) .
$$

The proof of Theorem 6.14 will consist of a series of lemmas which will comprise the rest of this section.

Lemma 6.16. Suppose $1 /(p-1) \leq b^{\prime} \leq b \leq p /(p-1)$. Set $e^{\prime}:=b^{\prime}-\frac{1}{p-1}$. If

$$
\mathcal{N}\left(b^{\prime}, b ; 0\right) \subset V\left(b^{\prime}, b ; 0\right)+\mathcal{L}_{\pi \hat{f}_{t}} \mathcal{M}\left(b^{\prime}, b ; e^{\prime}\right)
$$

then

$$
\mathcal{N}\left(b^{\prime}, b ; 0\right) \subset V\left(b^{\prime}, b ; 0\right)+\partial \mathcal{M}\left(b^{\prime}, b ; e^{\prime}\right)
$$


Proof. On $\mathcal{K}\left(b^{\prime}, b\right)$ we may write $\partial:=t \frac{\partial}{\partial t}+W(t, x)$ where

$$
W(t, x):=\sum_{j=0}^{\infty} \pi_{j} p^{j} \hat{f}_{t}^{\tau^{j}}\left(t^{p^{j}}, x^{p^{j}}\right) .
$$

For each $j \in \mathbb{Z}_{\geq 0}$ write

$$
\hat{f}_{t}^{\tau^{j}}\left(t^{p^{j}}, x^{p^{j}}\right)=\hat{f}_{t}(t, x)^{p^{j}}+p g_{j}(t, x)
$$

for some polynomial $g_{j}$ whose coefficients lie in $\mathbb{Z}_{q}[\pi]$. This allows us to write

$$
W(t, x)=\pi \hat{f}_{t}(t, x) Q_{2}(t, x)+K_{2}(t, x)
$$

where

$$
\begin{aligned}
Q_{2}(t, x) & :=\sum_{j=0}^{\infty} \pi_{j} \pi^{-1} p^{j} \hat{f}_{t}(t, x)^{p^{j}-1} \\
K_{2}(t, x) & :=\sum_{j=1}^{\infty} \pi_{j} p^{j+1} g_{j}(t, x) .
\end{aligned}
$$

Using a similar argument as that in Lemma 6.5, one may show $Q_{2}, \frac{1}{Q_{2}}, K_{2} \in \mathcal{K}\left(\frac{p}{p-1}, \frac{p}{p-1} ; 0\right)$.

Since $t x \mid \hat{f}_{t}$, we may write $Q_{2}(t, x)=1+(t x)^{p-1} \nu(t, x)$ for some $\nu$, making $Q_{2}(t, x) x=x+t^{p-1} x^{p} \nu(t, x) \in$ $\mathcal{K}\left(\frac{p}{p-1}, \frac{p}{p-1} ;-\left(\frac{p}{p-1}\right) w_{0}(1)\right)$. By Lemma 6.5 , we may write

$$
t^{p-1} x^{p} \nu(t, x)=B_{1} x+\cdots+B_{d-1} x^{d-1}+\pi \hat{f}_{x}(t, x) h(t, x),
$$

where $B_{j} x^{j} \in \mathcal{K}\left(\frac{p}{p-1}, \frac{p}{p-1} ;-\left(\frac{p}{p-1}\right) w_{0}(1)\right)$ and $h \in \mathcal{K}\left(\frac{p}{p-1}, \frac{p}{p-1} ;-\left(\frac{p}{p-1}\right) w_{0}(1)+1\right)$. Furthermore, since $t^{p-1}$ divides the left-hand side, by Lemma 6.5 each $B_{j}$ for $j=1, \ldots, d-1$ is also divisible by $t^{p-1}$, as well as $h$.

From 21, since

$$
Q_{2}(t, x) x=\left(1+B_{1}\right) x+B_{2} x^{2}+\cdots+B_{d-1} x^{d-1}+\pi \hat{f}_{x}(t, x) h(t, x),
$$

we may write for each $i=1, \ldots, d-1$

$$
W(t, x) x^{i}=\left(1+B_{1}\right) \pi \hat{f}_{t}(t, x) x^{i}+S_{i}(t, x)+T_{i}(t, x)
$$

where

$$
\begin{aligned}
& S_{i}:=\pi \hat{f}_{t}\left(B_{2} x^{2}+\cdots+B_{d-1} x^{d-1}\right) x^{i-1} \\
& T_{i}:=K_{2} x^{i}+D(t)\left(Q_{1}^{-1} \pi \hat{f}_{t} h x^{i-1}\right)-x \frac{\partial}{\partial x}\left(Q_{1}^{-1} \pi \hat{f}_{t} h x^{i-1}\right)-K_{1} Q_{1}^{-1} \pi \hat{f}_{t} h x^{i-1} .
\end{aligned}
$$

From the estimates above one may show $T_{i} \in \mathcal{K}\left(\frac{p}{p-1}, \frac{p}{p-1} ;-\left(\frac{p}{p-1}\right) w_{0}(i)\right)$, and so $T_{i} \in \mathcal{K}\left(b^{\prime}, b ;-b w_{0}(i)\right)$. Furthermore, $t^{p} x$ divides each $S_{i}$ since $t$ divides $f_{t}$ and $t^{p-1}$ divides each $B_{j}$. Thus, $S_{i} \in \mathcal{K}\left(b^{\prime}, b ;-e^{\prime}+\varrho-b w_{0}(i)\right)$ where

$$
\varrho:=\left(\frac{p}{p-1}-b^{\prime}\right)\left(w_{1}(p)-1\right)+\left(\frac{p}{p-1}-b\right) w_{0}(1) .
$$

By Theorem 6.4 we may write

$$
\begin{aligned}
& S_{i}=C_{i, 1} x+\cdots+C_{i, d-1} x^{d-1}+D(t) z_{i} \\
& T_{i}=A_{i, 1} x+\cdots+A_{i, d-1} x^{d-1}+D(t) w_{i}
\end{aligned}
$$

where

$$
C_{i, j} \in L\left(b^{\prime} ; b\left(w_{0}(j)-w_{0}(i)\right)-e^{\prime}+\varrho\right) \quad \text { and } \quad A_{i, j} \in L\left(b^{\prime} ; b\left(w_{0}(j)-w_{0}(i)\right)\right) .
$$

These define $(d-1) \times(d-1)$ matrices $C:=\left(C_{i, j}\right)$ and $A:=\left(A_{i, j}\right)$. It follows from 25 that $C$ and $A$ define morphisms

$$
\begin{aligned}
& C: \mathcal{H}_{1, t}\left(b^{\prime}, b ; e^{\prime}\right) \rightarrow \mathcal{H}_{1, t}\left(b^{\prime}, b ; \varrho\right) \\
& A: \mathcal{H}_{1, t}\left(b^{\prime}, b ; 0\right) \rightarrow \mathcal{H}_{1, t}\left(b^{\prime}, b ; 0\right) .
\end{aligned}
$$


By Lemma 6.13 below this means

$$
\begin{aligned}
& \mathcal{L}_{C}: \mathcal{H}_{1, t}^{(k)}\left(b^{\prime}, b ; e^{\prime}\right) \rightarrow \mathcal{H}_{1, t}^{(k)}\left(b^{\prime}, b ; \varrho\right) \\
& \mathcal{L}_{A}: \mathcal{H}_{1, t}^{(k)}\left(b^{\prime}, b ; 0\right) \rightarrow \mathcal{H}_{1, t}^{(k)}\left(b^{\prime}, b ; 0\right) .
\end{aligned}
$$

Finally, with $\mu:=1+B_{1}$, from $(22)$ and (24) we see that

$$
\mathcal{L}_{W}=\mu \mathcal{L}_{\pi \hat{f}_{t}}+\mathcal{L}_{C}+\mathcal{L}_{A}
$$

We will first suppose $\frac{1}{p-1}<b^{\prime}<\frac{p}{p-1}$. Let $\xi \in \mathcal{N}\left(b^{\prime}, b ; 0\right)$. By the hypothesis on $\mathcal{L}_{\pi \hat{f}_{t}}$, there exists $\eta_{1} \in$ $V\left(b^{\prime}, b ; 0\right)$ and $\zeta_{1} \in \mathcal{M}\left(b^{\prime}, b ; e^{\prime}\right)$ so that $\xi=\eta_{1}+\mathcal{L}_{\pi \hat{f}_{t}} \zeta_{1}$. Hence,

$$
\begin{aligned}
\xi & =\eta_{1}+\left(\mathcal{L}_{C}+\mathcal{L}_{A}\right)\left(-\mu^{-1} \zeta_{1}\right)+\mathcal{L}_{W}\left(\mu^{-1} \zeta_{1}\right) \\
& =\eta_{1}+\underbrace{\left(\mathcal{L}_{C}+\mathcal{L}_{A}+t \frac{d}{d t}\right)\left(-\mu^{-1} \zeta_{1}\right.}_{=: \nu_{1}})+\partial(\underbrace{\mu^{-1} \zeta_{1}}_{=: \zeta_{1}^{\prime}}),
\end{aligned}
$$

and so $\xi=\eta_{1}+\nu_{1}+\partial \zeta_{1}^{\prime}$. By our assumptions on $b^{\prime}$ we have $\varrho>0$. Let $\epsilon:=\min \left\{\varrho, e^{\prime}\right\}>0$. Notice that $\nu_{1} \in \mathcal{H}_{1, t}^{(k)}\left(b^{\prime}, b ; \epsilon\right)$ and $\zeta_{1}^{\prime} \in \mathcal{H}_{1, t}^{(k)}\left(b^{\prime}, b ; e^{\prime}\right)$. We have just taken $\xi$ and written it as the sum $\eta_{1}+\nu_{1}+\partial \zeta_{1}^{\prime}$. We may now do the same procedure but now with $\nu_{1}$, which means

$$
\xi=\left(\eta_{1}+\eta_{2}\right)+\nu_{2}+\partial\left(\zeta_{1}^{\prime}+\zeta_{2}^{\prime}\right)
$$

Continuing this process, we are led to an equation of the form

$$
\xi=\left(\eta_{1}+\cdots+\eta_{N}\right)+\nu_{N}+\partial\left(\zeta_{1}^{\prime}+\cdots+\zeta_{N}^{\prime}\right)
$$

where $\eta_{i} \in V\left(b^{\prime}, b ;(i-1) \epsilon\right), \zeta_{i}^{\prime} \in \mathcal{H}_{1, t}^{(k)}\left(b^{\prime}, b ;(i-1) \epsilon+e^{\prime}\right)$, and $\nu_{N} \in \mathcal{N}\left(b^{\prime}, b ; N \epsilon\right)$. Letting $N$ tend to infinity, we see that

$$
\xi=\eta+\partial \zeta^{\prime}
$$

where $\eta:=\sum_{i=1}^{\infty} \eta_{i} \in V\left(b^{\prime}, b ; 0\right)$ and $\zeta^{\prime}:=\sum_{i=1}^{\infty} \zeta_{i}^{\prime} \in \mathcal{M}\left(b^{\prime}, b ; e^{\prime}\right)$ as desired.

Suppose now that $b=b^{\prime}=\frac{p}{p-1}$. Let $\xi \in \mathcal{N}\left(b^{\prime}, b ; 0\right)$. Let $0<\epsilon<1$. Then $\xi \in \mathcal{N}\left(b^{\prime}-\epsilon, b ; 0\right)$. By the above argument, there exists $\eta^{(\epsilon)} \in V\left(b^{\prime}-\epsilon, b ; 0\right)$ and $\zeta^{(\epsilon)} \in \mathcal{M}\left(b^{\prime}-\epsilon, b ; 1-\epsilon\right)$ such that $\xi=\eta^{(\epsilon)}+\partial \zeta^{(\epsilon)}$. Restricting ourselves to a strictly decreasing sequence of $\epsilon$ which tend to zero constructs sequences $\left\{\eta^{(\epsilon)}\right\}_{\epsilon} \subset V\left(\frac{1}{p-1}, b ; 0\right)$ and $\left\{\zeta^{(\epsilon)}\right\}_{\epsilon} \subset \mathcal{M}\left(\frac{1}{p-1}, b ; 0\right)$. These two spaces are compact in the topology of coefficientwise convergence, and so the sequences have accumulation points $\eta$ and $\zeta$, respectively. By construction, $\eta$ belongs to each $V\left(b^{\prime}-\epsilon, b ; 0\right)$ for every $\epsilon>0$, which forces $\eta \in V\left(b^{\prime}, b ; 0\right)$. A similar argument holds for $\zeta$, showing $\zeta$ is an element of $\mathcal{M}\left(b^{\prime}, b ; 1\right)$. This proves the result for the case $b^{\prime}=b=\frac{p}{p-1}$.

Lastly, suppose $b^{\prime}=\frac{1}{p-1}$. For each $N \in \mathbb{Z}_{\geq 0}$, we may write

$$
\xi=\sum_{\mathbf{i}}\left(\sum_{n=1}^{N} B_{n, \mathbf{i}} t^{n}\right) e^{\mathbf{i}}+\sum_{\mathbf{i}}\left(\sum_{n=N+1}^{\infty} B_{n, \mathbf{i}} t^{n}\right) e^{\mathbf{i}} .
$$

Let $\epsilon>0$. For each $1 \leq n \leq N$, since $b^{\prime} w_{1}(n)+b w_{0}(\mathbf{i}) \geq\left(b^{\prime}+\frac{\epsilon}{w_{1}(N)}\right) w_{1}(n)+b w_{0}(\mathbf{i})-\epsilon$, we see that

$$
\sum_{\mathbf{i}}\left(\sum_{n=1}^{N} B_{n, \mathbf{i}} t^{n}\right) e^{\mathbf{i}} \in \mathcal{N}\left(b^{\prime}+\frac{\epsilon}{w_{1}(N)}, b ;-\epsilon\right) .
$$

Suppose $b \geq b^{\prime}+\frac{\epsilon}{w_{1}(N)}>\frac{1}{p-1}$. It follows from the argument above that there exists $\eta^{(\epsilon, N)} \in V\left(b^{\prime}+\frac{\epsilon}{w_{1}(N)}, b ;-\epsilon\right)$ and $\zeta^{(\epsilon, N)} \in \mathcal{M}\left(b^{\prime}+\frac{\epsilon}{w_{1}(N)}, b ;-\epsilon+\frac{\epsilon}{w_{1}(N)}\right)$ such that

$$
\sum_{\mathbf{i}}\left(\sum_{n=1}^{N} B_{n, \mathbf{i}} t^{n}\right) e^{\mathbf{i}}=\eta^{(\epsilon, N)}+\partial \zeta^{(\epsilon, N)} .
$$

We have just constructed sequences $\left\{\eta^{(\epsilon, N)}\right\}_{N=1}^{\infty} \subset V\left(b^{\prime}, b ;-\epsilon\right)$ and $\left\{\zeta^{(\epsilon, N)}\right\}_{N=1}^{\infty} \subset \mathcal{M}\left(b^{\prime}, b ;-\epsilon\right)$. Since both of these spaces are compact in the topology of coefficientwise convergence we may restrict ourselves to convergent subsequences with limits $\eta^{(\epsilon)} \in V\left(b^{\prime}, b ;-\epsilon\right)$ and $\zeta^{(\epsilon)} \in \mathcal{M}\left(b^{\prime}, b ;-\epsilon\right)$ which satisfy

$$
\xi=\eta^{(\epsilon)}+\partial \zeta^{(\epsilon)}
$$


In the coefficientwise convergence topology, letting $\epsilon \rightarrow 0^{+}$, there exists $\eta \in V\left(b^{\prime}, b ; 0\right)$ and $\zeta \in \mathcal{M}\left(b^{\prime}, b ; 0\right)$ such that

$$
\xi=\lim _{\epsilon \rightarrow 0^{+}} \eta^{(\epsilon)}+\partial \zeta^{(\epsilon)}=\eta+\partial \zeta
$$

This finishes the case when $b>b^{\prime}=\frac{1}{p-1}$. The case when $b=b^{\prime}=\frac{1}{p-1}$ is similar.

Lemma 6.17. Suppose $\frac{1}{p-1}<b^{\prime} \leq \frac{p}{p-1}$, and $1 /(p-1) \leq b \leq p /(p-1)$. Suppose

$$
V\left(b^{\prime}, b\right) \cap \mathcal{L}_{\pi \hat{f}_{t}} \mathcal{M}\left(b^{\prime}, b\right)=\{0\}
$$

Then

$$
V\left(b^{\prime}, b\right) \cap \partial \mathcal{M}\left(b^{\prime}, b\right)=\{0\} .
$$

Proof. We will first assume $\frac{p}{p-1}>b^{\prime}>\frac{1}{p-1}$. Let $\eta \in V\left(b^{\prime}, b\right) \cap \partial \mathcal{M}\left(b^{\prime}, b\right)$ be non-zero, and let $\xi \in \mathcal{M}\left(b^{\prime}, b\right)$ be such that $\partial \xi=\eta$. Let $c$ be a real number such that $\xi \in \mathcal{M}\left(b^{\prime}, b ; c\right)$ but $\xi \notin \mathcal{M}\left(b^{\prime}, b ; c+\epsilon\right)$ for any $\epsilon>0$. We will prove that no such $c$ exists, contradicting the existence of a non-zero $\eta$.

Using notation from the proof of Lemma 6.16, we have $\partial=t \frac{d}{d t}+\mu \mathcal{L}_{\pi \hat{f}_{t}}+\mathcal{L}_{C}+\mathcal{L}_{A}$, and so

$$
\eta=\partial \xi=\mu \mathcal{L}_{\pi \hat{f}_{t}} \xi+\underbrace{\left(t \frac{d}{d t}+\mathcal{L}_{C}+\mathcal{L}_{A}\right) \xi}_{=: \zeta_{1}^{\prime}} .
$$

Letting $\epsilon:=\min \left\{\varrho, e^{\prime}\right\}>0$, where $\varrho$ comes from $(23)$, we see that $\zeta_{1}^{\prime} \in \mathcal{M}\left(b^{\prime}, b ; c-e^{\prime}+\epsilon\right)$. From Lemma 6.16, there exists $\eta_{1} \in V\left(b^{\prime}, b ; c-e^{\prime}+\epsilon\right)$ and $\zeta_{1} \in \mathcal{M}\left(b^{\prime}, b ; c+\epsilon\right)$ such that

$$
\begin{aligned}
\zeta_{1}^{\prime} & =\eta_{1}+\partial \zeta_{1} \\
& =\eta_{1}+\mathcal{L}_{\pi \hat{f}_{t}}\left(\mu \zeta_{1}\right)+\underbrace{\left(t \frac{d}{d t}+\mathcal{L}_{C}+\mathcal{L}_{A}\right) \zeta_{1}}_{=: \zeta_{2}^{\prime}} .
\end{aligned}
$$

Observe that $\zeta_{2}^{\prime} \in \mathcal{M}\left(b^{\prime}, b ; c+2 \epsilon-e^{\prime}\right)$, and

$$
\eta=\mathcal{L}_{\pi \hat{f}_{t}}\left(\mu \xi+\mu \zeta_{1}\right)+\zeta_{2}^{\prime}+\eta_{1}
$$

Iterating this process, but now starting with $\zeta_{2}^{\prime}$, we are led to the equation

$$
\eta=\mathcal{L}_{\pi \hat{f}_{t}}\left(\mu\left(\xi+\zeta_{1}+\cdots+\zeta_{N}\right)\right)+\zeta_{N+1}^{\prime}+\left(\eta_{1}+\cdots+\eta_{N}\right)
$$

with $\zeta_{i} \in \mathcal{M}\left(b^{\prime}, b ; c+i \epsilon\right), \zeta_{N+1}^{\prime} \in \mathcal{M}\left(b^{\prime}, b ; c+(N+1) \epsilon-e^{\prime}\right)$, and $\eta_{i} \in V\left(b^{\prime}, b ; c-e^{\prime}+i \epsilon\right)$. Hence,

$$
\eta-\sum_{i=1}^{\infty} \eta_{i}=\mathcal{L}_{\pi \hat{f}_{t}}\left(\mu\left(\xi+\sum_{i=1}^{\infty} \zeta_{i}\right)\right) .
$$

It follows from the hypothesis on $\mathcal{L}_{\pi \hat{f}_{t}}$ that $\xi=-\sum_{i=1}^{\infty} \zeta_{i} \in \mathcal{M}\left(b^{\prime}, b ; c+\epsilon\right)$, contradicting out choice of $c$.

The result follows for $b^{\prime}=\frac{p}{p-1}$ since

$$
V\left(b^{\prime}, b\right) \cap \partial \mathcal{M}\left(b^{\prime}, b\right) \subset V\left(b^{\prime}-\epsilon, b\right) \cap \partial \mathcal{M}\left(b^{\prime}-\epsilon, b\right)=\{0\},
$$

where the last equality follows for any $\epsilon>0$ by the preceding argument.

\section{References}

[1] Ahmed Abbes and Takeshi Saito. Local Fourier transform and epsilon factors. arXiv:0809.0180v1 [math.AG], 2008 .

[2] Adolphson and Sperber. Newton polyhedra and the degree of the $L$-function associated to an exponential sum. Invent. Math., 88:555-569, 1987.

[3] Alan Adolphson. A p-adic theory of Hecke polynomials. Duke Math. J., 43(1):115-145, 1976.

[4] Alan Adolphson. On the distribution of angles of Kloosterman sums. J. Reine. Angew. Math., 395:214-220, 1989. 
[5] Laurent Clozel, Michael Harris, and Richard Taylor. Automorphy for some $l$-adic lifts of automorphic mod $l$ Galois representations. Publ. Math. Inst. Hautes Études Sci., (108):1-181, 2008. With Appendix A, summarizing unpublished work of Russ Mann, and Appendix B by Marie-France Vignéras.

[6] Pierre Deligne. Formes modulaires et reprsentations l-adiques. Séminaire Bourbaki, 11(355):34, 1968.

[7] Pierre Deligne. Application de la formule des traces aux sommes trigonométriques, volume 569 of Lecture Notes in Mathematics, pages 168-232. Springer-Verlag, 1977.

[8] Pierre Deligne. Dualité, volume 569 of Lecture Notes in Mathematics, pages 154-167. Springer-Verlag, 1977.

[9] B. Dwork. On the zeta function of a hypersurface: II. Annals of Math, 80(2):227-299, Sep. 1964.

[10] Bernard Dwork. On Hecke polynomials. Inventiones math., 12:249-256, 1971.

[11] Lei Fu. Calculation of $\ell$-adic Local Fourier Transformations. arXiv: 0702436 [math.AG], 2007.

[12] Lei Fu and Daqing Wan. L-functions for symmetric products of Kloosterman sums. J. Reine Angew. Math., 589:79 - 103, 2005.

[13] Lei Fu and Daqing Wan. Trivial factors for $L$-functions of symmetric products of Kloosterman sheaves. Finite Fields Appl., 14(2):549-570, 2008.

[14] F. Gouvêa and B. Mazur. Families of modular eigenforms. Math. Comp., 58(198):793-805, 1992.

[15] C. Douglas Haessig. L-functions of symmetric powers of cubic exponential sums. J. Reine Angew. Math., 631:1-57, 2009.

[16] M. Harris, N. Shepherd Barron, and R. Taylor. A family of Calabi-yau varieties and potential automorphy. Annals of Math, (171):779-813, 2010.

[17] S. Hong. Newton polygons of L functions associated with exponential sums of polynomials of degree four over finite fields. Finite Fields and Their Applications, 7(1):205-237, 2001.

[18] Nicholas M. Katz. On the monodromy groups attached to certain families of exponential sums. Duke Math. J., 54(1):41-56, 1987.

[19] Nicholas M. Katz. Gauss Sums, Kloosterman Sums, and Monodromy Groups, volume 116 of Annals of Mathematics Studies. Princeton University Press, 1988.

[20] Nicholas M. Katz. Exponential Sums and Differential Equations, volume 124 of Annals of Mathematics Studies. Princeton University Press, 1990.

[21] Nicholas M. Katz. $G_{2}$ and Hypergeometric Sheaves. Finite Fields Appl., 13(2):175-223, 2007.

[22] Gérard Laumon. Transformation de Fourier, constantes d'équations fonctionnelles, et conjecture de Weil. Publ. Math. IHES, (65):131-210, 1987.

[23] Philippe Robba. Symmetric powers of the p-adic Bessel equation. J. Reine Angew. Math., 366:194 - 220, 1986.

[24] Jasper Scholten and Hui June Zhu. The first slope case of Wan's conjecture. Finite Fields Appl., 8(4):414-419, 2002.

[25] S. Sperber. On the p-adic theory of exponential sums. American Journal of Mathematics, 108(2):255-296, 1986.

[26] Ondrej Šuch. Monodromy of Airy and Kloosterman sheaves. Duke Mathematical Journal, 103(3):397-444, 2000.

[27] R. Taylor. Automorphy for some $\ell$-adic lifts of automorphic $\bmod \ell$ representations. II. Pub. Math. IHES, 108:183-239, 2008.

[28] D. Wan. Dimension variation of classical and p-adic modular forms. Invent. Math., 133:469-498, 1998.

[29] D. Wan. Dwork's conjecture on unit root zeta functions. Ann. Math., 150:867-927, 1999.

[30] D. Wan. Higher rank case of Dwork's conjecture. J. Amer. Math. Soc., 13:807-852, 2000.

[31] D. Wan. Rank one case of Dwork's conjecture. J. Amer. Math. Soc., 13:853-908, 2000. 